\title{
LOCAL MIRROR SYMMETRY AND THE SUNSET FEYNMAN INTEGRAL
}

\author{
by \\ Spencer Bloch, Matt Kerr \& Pierre Vanhove
}

\begin{abstract}
. -
We study the sunset Feynman integral defined as the scalar two-point self-energy at two-loop order in a two dimensional space-time.

We firstly compute the Feynman integral, for arbitrary internal masses, in terms of the regulator of a class in the motivic cohomology of a 1-parameter family of open elliptic curves. Using an Hodge theoretic (B-model) approach, we show that the integral is given by a sum of elliptic dilogarithms evaluated at the divisors determined by the punctures.

Secondly we associate to the sunset elliptic curve a local non-compact Calabi-Yau 3 -fold, obtained as a limit of elliptically fibered compact Calabi-Yau 3-folds. By considering the limiting mixed Hodge structure of the Batyrev dual A-model, we arrive at an expression for the sunset Feynman integral in terms of the local Gromov-Witten prepotential of the del Pezzo surface of degree 6. This expression is obtained by proving a strong form of local mirror symmetry which identifies this prepotential with the second regulator period of the motivic cohomology class.
\end{abstract}

IPHT-t15/135, DAMTP-2016-1. 


\section{Contents}

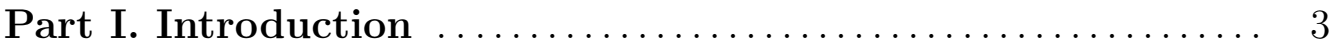

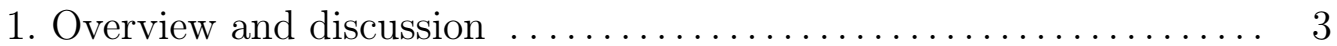

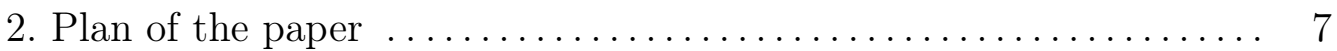

Part II. The elliptic dilogarithm $\ldots \ldots \ldots \ldots \ldots \ldots \ldots \ldots \ldots$

3. The sunset Feynman integral $\ldots \ldots \ldots \ldots \ldots \ldots \ldots \ldots \ldots \ldots$

3.1. The sunset open elliptic curve $\ldots \ldots \ldots \ldots \ldots \ldots \ldots \ldots . \ldots$

3.2. Derivation of the Picard-Fuchs equation $\ldots \ldots \ldots \ldots \ldots \ldots 11$

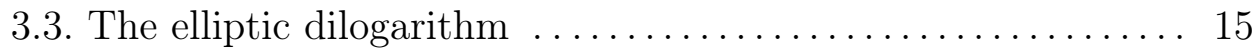

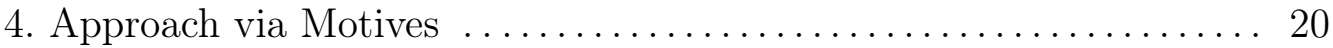

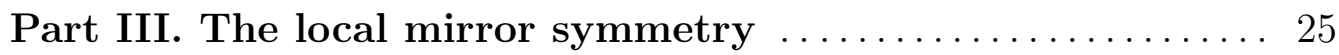

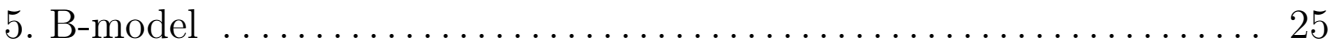

5.1. Laurent polynomial .......................... 25

5.2. Maximal projective crepant partial desingularization ..... 26

5.3. Elliptic fibration .............................. 28

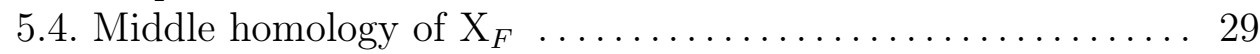

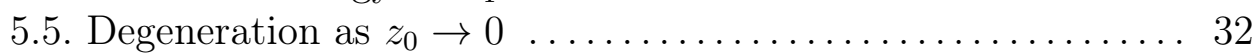

5.6. Middle cohomology of $\mathrm{X}_{0} \ldots \ldots \ldots \ldots \ldots \ldots \ldots \ldots \ldots \ldots \ldots \ldots \ldots$

5.7 . Monodromy and asymptotics of periods $\ldots \ldots \ldots \ldots \ldots \ldots$

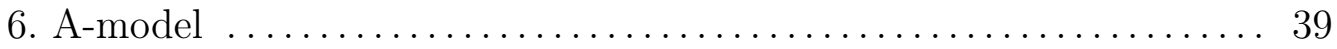

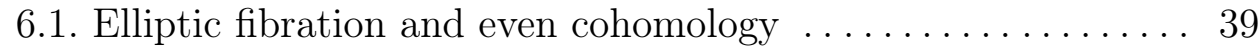

6.2. The quantum $\mathbb{Z}$-variation of Hodge structure $\ldots \ldots \ldots \ldots .41$

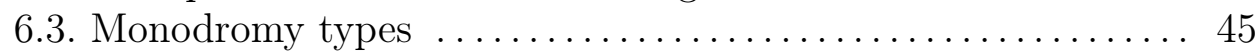

6.4. Mirror map .............................. 47

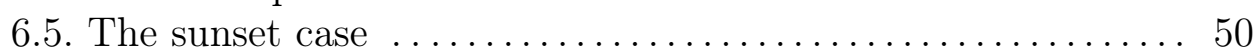

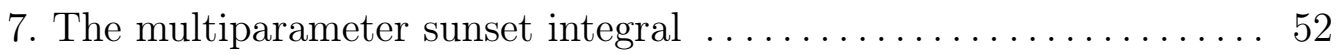

7.1. Degeneration of the Yukawa coupling ............. 52

7.2. Inhomogeneous equation for the sunset integral . . . . . . . 54

7.3. On the local Gromov-Witten numbers ................ 58

7.4. The local Gromov-Witten numbers in the

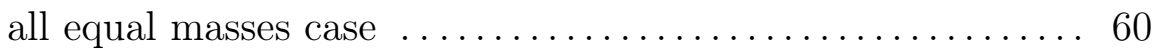

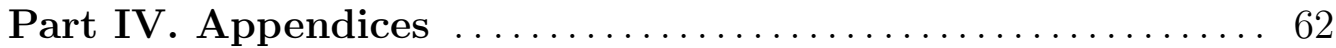

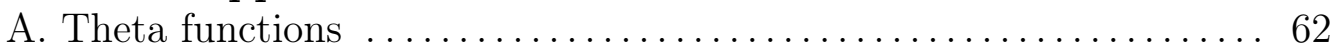

B. The coefficients of the Picard-Fuchs equation $\ldots \ldots \ldots \ldots \ldots \ldots 63$

B.1. The coefficients $C_{x}, C_{y}$ and $C_{z} \ldots \ldots \ldots \ldots \ldots \ldots \ldots \ldots$

B.2. The coefficients $\tilde{C}_{x}, \tilde{C}_{y}$ and $\tilde{C}_{z} \ldots \ldots \ldots \ldots \ldots \ldots \ldots \ldots$

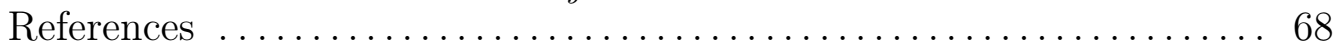




\section{PART I \\ INTRODUCTION}

\section{Overview and discussion}

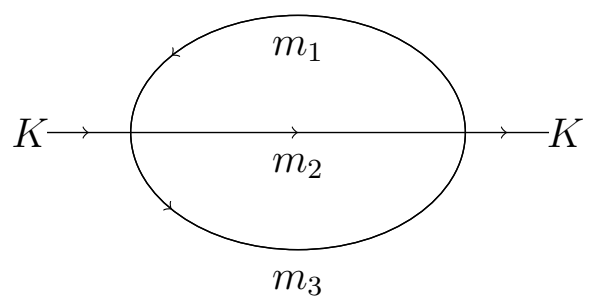

This work concerns the Feynman integral in two dimensional space-time associated to the sunset graph in the above figure, given by

$$
\mathcal{I}_{\ominus}(s):=s \int_{\substack{x \geq 0 \\ y \geq 0}} \frac{d x d y}{s\left(\xi_{1}^{2} x+\xi_{2}^{2} y+\xi_{3}^{2}\right)(x y+x+y)-x y} .
$$

Here $\xi_{i}=m_{i} / \mu(i=1,2,3)$ are positive non vanishing real numbers, given by the ratios of the internal masses by the arbitrary infrared scale $\mu$, and $s$ is the inverse of the norm of the external momentum $K^{2}=\mu^{2} / s$. (See [BW, BV, Va] for a derivation of (1.1) from the usual Feynman representation.)

This integral is a multivalued function of $s$ on $\mathbb{C} \backslash\left[\left(\xi_{1}+\xi_{2}+\xi_{3}\right)^{-2},+\infty[\right.$. In general, the multivalueness of the Feynman integral plays an important role in physics, as this is imposed by unitarity of quantum field theory [EH]. A large class of Feynman integrals can be easily determined from their differential equations [AM, RT, Henna, L, Hennb, T], and more generally are associated to motivic period integrals [B1, B2].

The geometry of the graph hypersurface is a family of elliptic curves

$$
\mathcal{E}_{\ominus}:=\left\{x y z-s\left(\xi_{1}^{2} x+\xi_{2}^{2} y+\xi_{3}^{2} z\right)(x y+x z+y z) \mid(x, y, z) \in \mathbb{P}^{2}\right\} .
$$

The structure of the motive associated to (1.1), discussed in sections 4 and 7.2, differs from the one given in the single masses case in [BV], because we now have a family of open elliptic curves, no longer modular, and the motive has a Kummer extension quotient.

We show that the sunset Feynman integral is given by (see section 3.3.2)(1)

$$
\mathcal{I}_{\ominus}(s) \equiv \frac{\mathbf{i} \varpi_{r}}{\pi}\left(\hat{E}_{2}\left(\frac{x\left(P_{1}\right)}{x\left(P_{2}\right)}\right)+\hat{E}_{2}\left(\frac{x\left(P_{2}\right)}{x\left(P_{3}\right)}\right)+\hat{E}_{2}\left(\frac{x\left(P_{3}\right)}{x\left(P_{1}\right)}\right)\right) \quad \bmod \text { periods }
$$

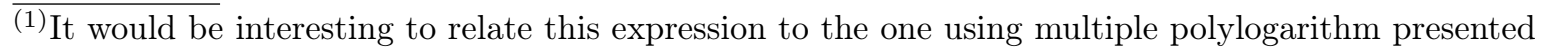
in [ABW2, ABW3, ABW4, ABW5]. 
where $\hat{E}_{2}(x)$ is the elliptic dilogarithm

$$
\hat{E}_{2}(x)=\sum_{n \geq 0}\left(\operatorname{Li}_{2}\left(q^{n} x\right)-\operatorname{Li}_{2}\left(-q^{n} x\right)\right)-\sum_{n \geq 1}\left(\operatorname{Li}_{2}\left(q^{n} / x\right)-\operatorname{Li}_{2}\left(-q^{n} / x\right)\right) .
$$

In (1.2), $\hat{E}_{2}$ is evaluated at the ratios of the images of the points $P_{1}:=[1: 0: 0]$, $P_{2}:=[0: 1: 0]$ and $P_{3}:=[0: 0: 1]$ in $\mathbb{C}^{\times} / q^{\mathbb{Z}}$, where $\log (q) /(2 \pi \mathbf{i})$ is the complex structure given by the period ratio of the elliptic curve; and $\varpi_{r}$ is the elliptic curve period which is real on the line $s>\left(\xi_{1}+\xi_{2}+\xi_{3}\right)^{-2}$.

The elliptic dilogarithm $\hat{E}_{2}(x)$ is not invariant under $x \rightarrow x q$ (see equation (3.70)), reflecting the multivalued nature of the Feynman integral. This was already the case for elliptic polylogarithm expansions of the Feynman integrals for the two-loop sunset $[\mathrm{BV}]$ and three-loop banana [BKV] with equal masses. The result in (1.2) generalizes the expression for the all equal masses case $\xi_{1}=\xi_{2}=\xi_{3}=1$ given in terms of elliptic dilogarithm in [BV].

The motivic approach in section 4 shows how the theory of motives can yield information about Feynman integrals. In general, the motive associated to a Feynman integral will depend on a family of hypersurfaces $X_{m, q} \subset \mathbb{P}^{n}$ depending on masses $m$ and external momenta $q$. The motive at $(m, q)$ is associated to the cohomology group $H^{n}\left(\mathbb{P}^{n}-X_{m, q}, \Delta\right)$ where $\Delta$ is the simplex defined by the vanishing of the product of the homogeneous coordinates. A general motivic analysis would begin by a study of $X_{m, q} \cap \Delta$. In simple cases like the sunset, this intersection is manageable and we are able to prove a duality

$$
H^{n}\left(\mathbb{P}^{n}-X_{m, q}, \Delta\right) \cong H^{n}\left(\mathbb{P}^{n}-\Delta, X_{m, q}\right)(n)^{\vee} .
$$

The motive on the right is related to the Milnor symbol $\left\{x_{1}, \ldots, x_{n}\right\}$ on $X_{m, q} \cap \mathbb{G}_{m}^{n}$, where the $x_{i}$ are the Laurent coordinates on $\mathbb{P}^{n}-\Delta=\mathbb{G}_{m}^{n}$. In the sunset case, this approach identifies the amplitude with an elliptic dilogarithm. A similar attack may be possible for more general graphs, though the above duality will no longer be perfect. The challenge will be to understand the role played by the structure at infinity $X_{m, q} \cap \Delta$.

In part III, we revisit the approach of [CKYZ] to local mirror symmetry, by semistably degenerating a family of elliptically-fibered Calabi-Yau 3-folds $\mathrm{X}_{z_{0}, \underline{z}}$ (defined by (5.1)) to a singular compactification $\mathrm{X}_{0, \underline{z}}$ of the local Hori-Vafa 3-fold

$$
Y_{\underline{z}}:=\left\{1-s\left(\xi_{1}^{2} x+\xi_{2}^{2} y+\xi_{3}^{2}\right)\left(1+x^{-1}+y^{-1}\right)+u v=0\right\} \subset\left(\mathbb{C}^{*}\right)^{2} \times \mathbb{C}^{2},
$$

and using the work of Iritani [Ir] to compare the asymptotic Hodge theory of this B-model to that of the mirror (elliptically fibered) A-model Calabi-Yau X'

The bulk of section 5 is concerned with the proof of the isomorphism

$$
H_{\text {lim }}^{3}\left(\mathrm{X}_{z_{0}, \underline{z}}\right) \supseteq \operatorname{ker}\left(T_{0}-I\right) \cong H_{3}\left(Y_{\underline{z}}\right)(-3)
$$


of mixed Hodge structures (Theorem 5.3), and the explicit construction of bases for $H^{3}\left(\mathrm{X}_{z_{0}, \underline{z}}\right)$ resp. $H_{3}\left(Y_{\underline{z}}\right)$. This allows us to invoke (in section 5.7) results of ${ }^{(2)}$ [DK, $\S 5]$ to compute, in the $z_{0} \rightarrow 0$ limit, the invariant periods of $\mathrm{X}$ in terms of "regulator periods" $R_{0}^{(i)}, R_{1}$ associated to a family of algebraic $K_{2}$-classes on the sunset elliptic curve family $E_{\underline{z}}$.

In $\S 6$, we compute Iritani's quantum $\mathbb{Z}$-variation of Hodge structure on the even cohomology of the Batyrev mirror $\mathrm{X}^{\circ}$ of $\mathrm{X}$, writing the periods in terms of its GromovWitten invariants (section 6.2) and the monodromy transformations in terms of its intersection theory (section 6.3). (The monodromies $T_{i}$ are computed in greater detail than we need, as they will be used to provide geometric realizations of certain monodromy cones in the forthcoming work [KPR].) Like $\mathrm{X}, \mathrm{X}^{\circ}$ is elliptically fibered, over a toric Fano surface $\mathbb{P}_{\Delta^{\circ}}$, which (for the sunset case) is just the del Pezzo of degree 6. Under the mirror map $\underline{z} \mapsto q(\underline{z})=e^{2 \pi \mathbf{i} \underline{\tau}(\underline{z})}$ (computed in section 6.4), we have the isomorphism of A- and B-model $\mathbb{Z}$-variation of Hodge structure

$$
H^{3}\left(\mathrm{X}_{z_{0}, \underline{z}}\right) \cong H^{\text {even }}\left(\mathrm{X}_{q_{0}, \underline{q}}^{\circ}\right) \text {, }
$$

and taking (the invariant part of) limiting mixed Hodge structure on both sides yields the relation

$$
2 \pi \mathbf{i} R_{1}=R_{0}^{(1)} R_{0}^{(2)}+R_{0}^{(2)} R_{0}^{(3)}+R_{0}^{(1)} R_{0}^{(3)}-\sum_{\substack{\ell_{1}+\ell_{2}+\ell_{3}=\ell>0 \\\left(\ell_{1}, \ell_{2}, \ell_{3}\right) \in \mathbb{N}^{3} \backslash(0,0,0)}} \ell N_{\ell_{1}, \ell_{2}, \ell_{3}} \prod_{i=1}^{3} Q_{i}^{\ell_{i}}
$$

between regulator periods and local Gromov-Witten numbers of $\mathbb{P}_{\Delta^{\circ}}$ (Corollary 6.3). The expression is done with respect to the local Kähler moduli $Q_{i}=e^{R_{0}^{(i)}}=\xi_{i}^{2} \hat{Q}$ for $i=1,2,3$ with $\hat{Q}=\exp \left(\hat{R}_{0}\right)$ and where $\hat{R}_{0}$ is the logarithmic Mahler measure

$$
\hat{R}_{0}=\mathbf{i} \pi-\int_{|x|=|y|=1} \log \left(s^{-1}-\left(\xi_{1}^{2} x+\xi_{2}^{2} y+\xi_{3}^{2}\right)\left(x^{-1}+y^{-1}+1\right)\right) \frac{d \log x d \log y}{(2 \pi \mathbf{i})^{2}} .
$$

That is, we prove that $R_{1}$ is the local Gromov-Witten prepotential of $\mathbb{P}_{\Delta^{\circ}}$, which is Conjecture $5.1^{(2)}$ of $[\mathrm{DK}]$; this puts the observations on asymptotics of the local Gromov-Witten invariants there (Corollary 5.3 of $[\mathrm{DK}]$ ) on a firm foundation at last.

All of what has just been described is carried out, in sections 6-5, in a greater level of generality so that the results described apply to other toric families of elliptic curves in addition to the sunset family.

The connection of all this to the Feynman integral (1.1) is given in section 7: writing $\omega_{\underline{z}}$ for a family of holomorphic 1-forms on $E_{\underline{z}}$, and $\left.R\right|_{E_{\underline{z}}}$ for the family of 1-currents associated to the family of algebraic $K_{2}$-classes, we have the equality

$$
\mathcal{I}_{\ominus}(s)=-\left.s \int_{E_{\underline{z}}} R\right|_{E_{\underline{z}}} \wedge \omega_{\underline{z}} .
$$

$\overline{(2)}$ The numbers of section, conjecture, theorem and equations refer to the published version of [DK]. 
Proposition 7.2 shows this leads to the inhomogeneous Picard-Fuchs equation for $\mathcal{I}_{\ominus}$ derived explicitly in section 3.3.

Remarkably we show that the sunset Feynman integral is given by the Legendre transform of the regulator period $\hat{R}_{1}=R_{1}$ (see (7.27) and (7.41))

$$
\mathcal{I}_{\ominus}(s) \simeq-s 2 \pi \mathbf{i} \pi_{0}\left(\frac{\partial \hat{R}_{1}}{\partial \hat{R}_{0}} \hat{R}_{0}-\hat{R}_{1}\right),
$$

which implies the expansion of the Feynman integral in terms of Gromov-Witten numbers (see sections 7.3 and 7.4)

$$
\mathcal{I}_{\ominus}(s)=-s^{2} \frac{\partial \hat{R}_{0}}{\partial s}\left(3 \hat{R}_{0}^{3}+\sum_{\substack{\ell_{1}+\ell_{2}+\ell_{3}=\ell>0 \\\left(\ell_{1}, \ell_{2}, \ell_{3}\right) \in \mathbb{N}^{3} \backslash(0,0,0)}} \ell(1-\ell \log \hat{Q}) N_{\ell_{1}, \ell_{2}, \ell_{3}} \prod_{i=1}^{3} \hat{Q}_{i}^{\ell_{i}}\right) .
$$

The local Gromov-Witten numbers $N_{\ell_{1}, \ell_{2}, \ell_{3}}$ can be expressed in terms of the virtual integer number of degree $\ell$ rational curves by

$$
N_{\ell_{1}, \ell_{2}, \ell_{3}}=\sum_{d \mid \ell_{1}, \ell_{2}, \ell_{3}} \frac{1}{d^{3}} n_{\frac{\ell_{1}}{d}, \frac{\ell_{2}}{d}, \frac{\ell_{3}}{d}}
$$

These numbers are tabulated in sections 7.3 and 7.4. In the particular case of the all equal masses case $\xi_{1}=\xi_{2}=\xi_{3}=1$, the mirror map gives (see section 7.4)

$$
\hat{Q}=-q \prod_{n \geq 1}\left(1-q^{n}\right)^{n \delta(n)} ; \quad \delta(n):=(-1)^{n-1}\left(\frac{-3}{n}\right)
$$

where $\left(\frac{-3}{n}\right)=0,1,-1$ for $n \equiv 0,1,2 \bmod 3$. The modularity of the family of sunset elliptic curves allows us to relates the sum of elliptic dilogarithms in $q$ of [BV] to the Gromov-Witten expansion in $\hat{Q}$, and implies the Legendre transform relation (1.5). Stienstra has already noticed in [St1, St2] the similarity between the mirror symmetry transformation in (1.7) and the ones between A-models of local Calabi-Yau and dimer models [ORV] for the topological vertex description of the Bmodel [AKMV, ADKMV]. Theorem 3.5 of [KOS] shows that the partition function of the dimer model is the Mahler measure of the Laurent polynomial defining the local Calabi-Yau model. In [St2] Stienstra constructed a dimer model associated to the all equal masses sunset elliptic curve $\xi_{1}=\xi_{2}=\xi_{3}=1$. In the case of unequal masses there is no modularity, and it is surprising that an analytic continuation of a sum of elliptic dilogarithms displays such relation to the local Gromov-Witten prepotential.

Special type of Feynman integrals for topological strings have been used to compute the local Gromov-Witten prepotential [Hor]. But our analysis leads to a different kind of result, firstly because the sunset Feynman integral is the Legendre transform (1.5) of the local Gromov-Witten prepotential, secondly because this Feynman graph is not obviously associated to world-sheet graphs of a topological string. Our results extend 
to the three-loop banana graph and the four-loop banana graph, leading to 4-fold and 5 -folds Calabi-Yau respectively (cf. section 5 of [DK]). The strong similarity of our analysis with the dimer models suggests that one could expect more connection between Gromov-Witten prepotential and (massive) quantum field theory Feynman integrals. We expect that this approach to Feynman integrals can shed some new light on the relation to string theory along the lines of the results of $[\mathrm{ABBF}]$.

\section{Plan of the paper}

The plan of the paper is the following. In part II, we analyse the sunset Feynman integral (1.1). In section 3.1 we describe the geometry of the sunset family of elliptic curve and in section 3.2 derive the Picard-Fuchs equation following Griffiths's approach in [Gri] for deriving the Picard-Fuchs equation from the cohomology of smooth projective hyperspace defined by rational form in $\mathbb{P}^{2}$. In section 3.3 we derive the expression (1.2) of the sunset integral in terms of elliptic dilogarithm. In section 3.3.1 we show how to reproduce the all equal masses result of [BV] and section 3.3.2 contains numerical verification of the three different masses case. We give a proof of these results using a motivic approach in section 4.

Part III of the paper deals with the mirror symmetry construction. In section 5 we describe the degeneration from a compact Calabi-Yau 3-fold $\mathrm{X}$ to the local HoriVafa model Y, and show in theorem 5.3 that the third homology of $\mathrm{Y}$ matches the invariant part of the limiting mixed Hodge structure of $H^{3}(\mathrm{X})$. In section 6 we describe the variation of Hodge structure arising on the A-model obtained by considering the Batyrev mirror of X. By comparing the limiting mixed Hodge structures of the Amodel and B-model, we prove in theorem 6.1 a strong form of local mirror symmetry - equality of variations of $\mathbb{Q}$-mixed Hodge structure. The particular case of the sunset integral is discussed in section 7.

In the appendix A we recall the main properties of Jacobi theta functions, and in the appendix B we give the detailed coefficients entering the derivation of the Picard-Fuchs equation in section 3.2.

\section{Acknowledgements}

The research of PV has received funding the ANR grant reference QST 12 BS05 003 01, and the CNRS grants PICS number 6430. PV is partially supported by a fellowship funded by the French Government at Churchill College, Cambridge, the European Research Council under the European Community's Seventh Framework Programme (FP7/2007-2013) ERC grant agreement no. [247252], and the STFC grant ST/L000385/1. MK was partially supported by NSF Grant DMS-1361147, and by the Fund for Mathematics. 


\section{PART II \\ THE ELLIPTIC DILOGARITHM}

\section{The sunset Feynman integral}

The sunset Feynman integral is

$$
\mathcal{I}_{\ominus}(s)=-s \int_{\Delta} \Omega_{\ominus}(s) .
$$

where the domain of integration is

$$
\Delta=\left\{(x, y, z) \in \mathbb{P}^{2} \mid x, y, z \geq 0\right\}
$$

and

$$
\Omega_{\ominus}(s):=\frac{x d y \wedge d z+y d z \wedge d x+z d x \wedge d y}{x y z\left(1-s \phi_{\ominus}\right)}
$$

where we have set

$$
\phi_{\ominus}=\left(\xi_{1}^{2} x+\xi_{2}^{2} y+\xi_{3}^{2} z\right)\left(x^{-1}+y^{-1}+z^{-1}\right) .
$$

Where $\xi_{i}=m_{i} / \mu$ for $i=1,2,3$ are non-vanishing positive real numbers given by the ratio of the internal masses parameters $m_{i}$ and an infrared scale $\mu$. In this work we assume that none of the masses vanish. As function of $1 / s:=K^{2} / \mu^{2}$ the integral is a multivalued function on the complex plane minus a line $\mathbb{C} \backslash\left[\left(\xi_{1}+\xi_{2}+\xi_{3}\right)^{2},+\infty[\right.$.

In this first part of the paper we show that this integral is an elliptic dilogarithm. We give to derivations on by a direct computation and second one based a motivic analysis.

3.1. The sunset open elliptic curve. - For generic values of the parameters the polar part of $\Omega_{\ominus}(s)$ defines an open with marked points elliptic curve

$$
\mathcal{E}_{\ominus}:=\left\{x y z-s\left(\xi_{1}^{2} x+\xi_{2}^{2} y+\xi_{3}^{2} z\right)(x y+x z+y z)=0 \mid(x, y, z) \in \mathbb{P}^{2}\right\} .
$$

The discriminant is

$$
\Delta=16 s^{-6} M_{6}^{2} \prod_{i=1}^{4}\left(1-s \mu_{i}^{2}\right)
$$

and the $J$-invariant is

$$
J=-\frac{\left(\prod_{i=1}^{4}\left(1-s \mu_{i}^{2}\right)+16 s^{3} \prod_{i=1}^{3} \xi_{i}^{2}\right)^{3}}{s^{6} M_{6}^{2} \prod_{i=1}^{4}\left(1-s \mu_{i}^{2}\right)}
$$

with

$$
\begin{aligned}
& \mu_{1}:=-\xi_{1}+\xi_{2}+\xi_{3}, \quad \mu_{2}:=\xi_{1}-\xi_{2}+\xi_{3}, \\
& \mu_{3}:=\xi_{1}+\xi_{2}-\xi_{3}, \quad \mu_{4}:=\xi_{1}+\xi_{2}+\xi_{3},
\end{aligned}
$$


and

$$
M_{2}:=\xi_{1}^{2}+\xi_{2}^{2}+\xi_{3}^{2}, M_{4}:=\xi_{1}^{2} \xi_{2}^{2}+\xi_{1}^{2} \xi_{3}^{2}+\xi_{2}^{2} \xi_{3}^{2}, M_{6}:=\xi_{1}^{2} \xi_{2}^{2} \xi_{3}^{2}
$$

For generic values of the masses $\xi_{1} \neq \xi_{2} \neq \xi_{3}$ there are six singular fibers: at $s=0$ of type $I_{6}$, at $s=\infty$ of type $I_{2}$ and for $1 \leq i \leq 4$ at $s^{-1}=\mu_{i}$ of type $I_{1}$.

We recall that for the all equal masses case $\xi_{1}=\xi_{2}=\xi_{3}=1$ there are only four singular fibers of type $I_{2}$ for $s=\infty, I_{3}$ for $s=1, I_{9}$ for $s=1 / 9$ and $I_{6}$ for $s=0[\mathrm{BV}]$.

If we introduce the Hauptmodul $u$

$$
u:=\frac{\left(1-s M_{2}\right)^{2}-4 s^{2} M_{4}}{\sqrt{16 s^{3} M_{6}}}
$$

the $J$-invariant takes the form

$$
J:=256 \frac{\left(3-u^{2}\right)^{3}}{4-u^{2}} .
$$

We introduce $q=\exp (2 \pi \mathbf{i} \tau)$ with $\tau=\varpi_{c} / \varpi_{r}$ the ratio of the complex period $\varpi_{c}$ and period $\varpi_{r}$ is the real period on the real axis $s>\left(\xi_{1}+\xi_{2}+\xi_{3}\right)^{-2}$. We assume that $\varpi_{c}$ has a positive imaginary part so that $|q|<1$ and $\tau$ is in the upper half-plane.

From the usual parametrization of the $J$-invariant in terms of theta-functions (see Appendix A) we deduce that the Hauptmodul $u$ is given by the three roots

$$
u_{a, b} \in\left\{u_{3,4}=\frac{\theta_{3}^{4}+\theta_{4}^{4}}{\theta_{3}^{2} \theta_{4}^{2}}, u_{2,3}=-\frac{\theta_{3}^{4}+\theta_{2}^{4}}{\theta_{3}^{2} \theta_{2}^{2}}, u_{2,4}=i \frac{\theta_{2}^{4}-\theta_{4}^{4}}{\theta_{2}^{2} \theta_{4}^{2}}\right\} .
$$

The action of $S L(2, \mathbb{Z})$ leaves invariant the $J$-invariant but rotates the three roots. The subgroup $\Gamma$ of $S L(2, \mathbb{Z})$ generated by $\tau \rightarrow \tau+2$ and $\tau \rightarrow \tau /(1-2 \tau)$ (see [Chand])

$$
\Gamma=\left\{\left(\begin{array}{ll}
a & b \\
c & d
\end{array}\right) \in S L(2, \mathbb{Z}) \mid\left(\begin{array}{ll}
a & b \\
c & d
\end{array}\right)=\left(\begin{array}{ll}
1 & 0 \\
0 & 1
\end{array}\right) \bmod 2\right\},
$$

leaves invariant the square of each individual roots $u_{a, b}^{2}$ for given $a, b$.

For each pair $(a, b)$ labelling the Hauptmodul in (3.12) the real period $\varpi_{r}$ is then given in terms of the theta constants (see Appendix $\mathrm{A}$ for definitions and conventions)

$$
\varpi_{r}=\pi \frac{\theta_{a} \theta_{b}}{\left(s^{-1} M_{6}\right)^{\frac{1}{4}}} .
$$

3.1.1. The points. - The intersection of the elliptic curve and the domain of integration $\Delta$ are the three points

$$
\partial \Delta \cap \mathcal{E}_{\ominus}=\left\{P_{1}=[1,0,0], P_{2}=[0,1,0], P_{3}=[0,0,1]\right\} .
$$

We will consider as well the other three points

$$
Q_{1}=\left[0,-\xi_{3}^{2}, \xi_{2}^{2}\right], \quad Q_{2}=\left[-\xi_{3}^{2}, 0, \xi_{1}^{2}\right], \quad Q_{3}=\left[-\xi_{2}^{2}, \xi_{1}^{2}, 0\right]
$$

arising from the intersection of the sunset elliptic curve and the lines defining the domain of integration $\Delta$. 
In order to map these points to $\mathcal{E}_{\ominus} \simeq \mathbb{C}^{\times} / q^{\mathbb{Z}}$ where $C^{\times}$is the multiplicative group of non-zero complex numbers, we use the following Weierstrass model for the sunset elliptic curve

$$
\zeta^{2} \eta=\sigma\left(s^{-1} M_{6} \eta^{2}+u \sqrt{s^{-1} M_{6}} \sigma \eta+\sigma^{2}\right) .
$$

For any choice of $(a, b, c)=(3,4,2),(2,3,4),(2,4,3)$, a point on the elliptic curve with coordinates $P=[\sigma, \zeta, \eta]$ and $\eta \neq 0$ is parametrized by ${ }^{(3)}$

$$
\begin{aligned}
& \frac{\sigma}{\eta}=\sqrt{s^{-1} M_{6}}\left(\Lambda_{a}(x)\right)^{2} \\
& \frac{\zeta}{\eta}=\left(s^{-1} M_{6}\right)^{\frac{3}{4}} \Lambda_{a}(x) M_{a, b, c}(x),
\end{aligned}
$$

where $x \in \mathbb{C}^{\times} / q^{\mathbb{Z}}$ and $\Lambda_{a}(x)$ and $M_{a, b, c}(x)$ are expressed in terms of the Jacobi theta functions defined in Appendix A

$$
\begin{aligned}
\Lambda_{a}(z) & :=\frac{\theta_{1}(x)}{\theta_{a}(x)} \\
M_{a, b, c}(z) & :=\frac{\theta_{c}^{2}}{\theta_{a} \theta_{b}} \frac{\theta_{a}(x) \theta_{b}(z)}{\left(\theta_{c}(x)\right)^{2}},
\end{aligned}
$$

that satisfy the relation

$$
\left(M_{a, b, c}(x)\right)^{2}=\left(\Lambda_{c}(x)\right)^{4}+u_{a, b}\left(\Lambda_{c}(x)\right)^{2}+1
$$

which is consequence of the Jacobi relations in (A.5) and in (A.6).

The differences of $P_{i j}:=P_{i}-P_{j}$ are mapped to

$$
\begin{aligned}
& P_{2,1}=\left[\xi_{1}^{2} \xi_{2}^{2},-\frac{\xi_{1}^{2} \xi_{2}^{2}}{2}\left(t-\xi_{1}^{2}-\xi_{2}^{2}+\xi_{3}^{2}\right), 1\right] \\
& P_{3,2}=\left[\xi_{2}^{2} \xi_{3}^{2},-\frac{\xi_{2}^{2} \xi_{3}^{2}}{2}\left(t+\xi_{1}^{2}-\xi_{2}^{2}-\xi_{3}^{2}\right), 1\right] \\
& P_{1,3}=\left[\xi_{1}^{2} \xi_{3}^{2},-\frac{\xi_{1}^{2} \xi_{3}^{2}}{2}\left(t-\xi_{1}^{2}+\xi_{2}^{2}-\xi_{3}^{2}\right), 1\right],
\end{aligned}
$$

that implies that for $(i, j, k)$ a permutation of $(1,2,3)$ and $c=2,3,4$

$$
\left(\frac{\theta_{1}\left(x\left(P_{i j}\right)\right)}{\theta_{c}\left(x\left(P_{i j}\right)\right)}\right)^{2}=\frac{\xi_{k} \sqrt{s^{-1}}}{\xi_{i} \xi_{j}}
$$

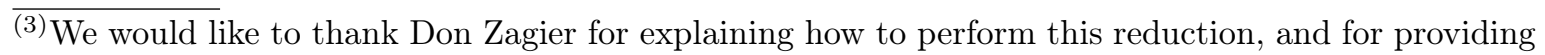
the key identities. 
The differences $Q_{i j}:=Q_{i}-P_{j}$ are mapped to

$$
\begin{aligned}
Q_{3,2} & =\left[\xi_{1}^{2} t, \frac{\xi_{1}^{2} t}{2}\left(s^{-1}+\xi_{1}^{2}-\xi_{2}^{2}-\xi_{3}^{2}\right), 1\right] \\
Q_{1,3} & =\left[\xi_{2}^{2} t, \frac{\xi_{2}^{2} t}{2}\left(s^{-1}-\xi_{1}^{2}+\xi_{2}^{2}-\xi_{3}^{2}\right), 1\right] \\
Q_{2,1} & =\left[\xi_{3}^{2} t, \frac{\xi_{3}^{2} t}{2}\left(s^{-1}-\xi_{1}^{2}-\xi_{2}^{2}+\xi_{3}^{2}\right), 1\right] .
\end{aligned}
$$

We then deduce that for $(i, j, k)$ a permutation of $(1,2,3)$ and $c=2,3,4$

$$
\left(\frac{\theta_{1}\left(x\left(Q_{i j}\right)\right)}{\theta_{c}\left(x\left(Q_{i j}\right)\right)}\right)^{2}=\frac{\xi_{i} \xi_{j}}{\sqrt{s^{-1}} \xi_{k}} .
$$

Using that $\theta_{1}(-x)=\theta_{2}(x)$ and $\theta_{3}(-x)=\theta_{4}(x)$, we find that $x\left(Q_{i j}\right)=-x\left(P_{i j}\right)$ for $i=1,2,3$. Implying that for $i=1,2,3$ we have $x\left(P_{i}\right) / x\left(Q_{i}\right)=-1 \in \mathbb{C}^{\times} / q^{\mathbb{Z}}$, which shows that the divisors $Q_{i}-P_{i}$ are of torsion two. This will play an important role when evaluating the elliptic dilogarithm in section 3.3.

3.2. Derivation of the Picard-Fuchs equation. - For completeness we give a short and explicit derivation of the differential equation satisfied by the sunset integral

$$
L_{\ominus}\left(-\frac{1}{s} \mathcal{I}_{\ominus}(s)\right)=S_{\ominus}(s)
$$

where $L_{\ominus}$ is the Picard-Fuchs operator (with $\delta_{s}:=s d / d s$ )

$$
L_{\ominus}=\delta_{s}^{2}+q_{1}(s) \delta_{s}+q_{0}(s)
$$

and $S_{\ominus}(s)$ is the inhomogeneous term composed by the sum of the Yukawa coupling $\mathcal{Y}_{\odot}(s)$ and logarithmic contributions in the masses

$$
S_{\ominus}(s)=\mathcal{Y}_{\ominus}(s)+\sum_{i=1}^{3} c_{i}(s) \log \left(\xi_{i}^{2}\right) .
$$

The logarithms terms arises from the Kummer quotient extension of the motive described in section 4 and in proposition 7.2.

This differential equation has already been derived in [RT, MSWZ]. We follow Griffiths' approach in [Gri] for deriving the Picard-Fuchs equation from the cohomology of smooth projective hyperspace defined by rational form in $\mathbb{P}^{2}$.

The action of the Picard-Fuchs operator on $\Omega_{\ominus}(s)$ is

$$
L_{\ominus} \Omega_{\ominus}(s)=\left(\frac{2(x y z)^{2}}{\Phi_{\ominus}^{3}}-\frac{\left(3-q_{1}(s)\right) x y z}{\Phi_{\ominus}^{2}}+\frac{1-q_{1}(s)+q_{0}(s)}{\Phi_{\ominus}}\right) \Omega
$$

with $\Omega=x d y \wedge d z+y d z \wedge d x+z d x \wedge d y$ and where we have set $\Phi_{\odot}=$ $x y z\left(1-s \phi_{\ominus}\right)$. 
For $C_{x}, C_{y}, C_{z}$ homogeneous polynomials of degree 4 in $(x, y, z)$ the one-form

$$
\beta_{1}=\frac{y C_{z}-z C_{y}}{\Phi_{\ominus}^{2}} d x+\frac{z C_{x}-x C_{z}}{\Phi_{\ominus}^{2}} d y+\frac{x C_{y}-y C_{x}}{\Phi_{\ominus}^{2}} d z
$$

satisfies $^{(4)}$

$$
d \beta_{1}=-2 \frac{\left(C_{x} \partial_{x}+C_{y} \partial_{y}+C_{z} \partial_{z}\right) \Phi_{\ominus}}{\Phi_{\ominus}^{3}} \Omega+\frac{\partial_{x} C_{x}+\partial_{y} C_{y}+\partial_{z} C_{z}}{\Phi_{\ominus}^{2}} \Omega .
$$

By choosing the polynomials $C_{x}, C_{y}$ and $C_{z}$ such that

$$
(x y z)^{2}=-\left(C_{x} \partial_{x}+C_{y} \partial_{y}+C_{z} \partial_{z}\right) \Phi_{\odot}
$$

then

$$
\frac{2(x y z)^{2}}{\Phi_{\ominus}^{3}} \Omega=-\frac{\partial_{x} C_{x}+\partial_{y} C_{y}+\partial_{z} C_{z}}{\Phi_{\ominus}^{2}} \Omega+d \beta_{1} .
$$

The expressions of the polynomials $C_{x}, C_{y}, C_{z}$ are given in Appendix B. We choose the coefficient $q_{1}(s)$ so that

$$
\left(\partial_{x} C_{x}+\partial_{y} C_{y}+\partial_{z} C_{z}\right)+\left(3-q_{1}(s)\right) x y z=\left(\tilde{C}_{x} \partial_{x}+\tilde{C}_{y} \partial_{y}+\tilde{C}_{z} \partial_{z}\right) \Phi_{\odot}
$$

where $\tilde{C}_{x}, \tilde{C}_{y}, \tilde{C}_{z}$ are at homogeneous polynomial of degree one in $(x, y, z)$, which detailed expressions are given in Appendix B. We find that $q_{1}(s)$ is given by

$$
q_{1}(s)=2+\sum_{i=1}^{4} \frac{1}{\mu_{i}^{2} s-1}-\frac{2 s M_{2}-6}{s^{2} \prod_{i=1}^{4} \mu_{i}-2 s M_{2}+3} .
$$

The one-form

$$
\beta_{2}=\frac{y \tilde{C}_{z}-z \tilde{C}_{y}}{\Phi_{\ominus}} d x+\frac{z \tilde{C}_{x}-x \tilde{C}_{z}}{\Phi_{\odot}} d y+\frac{x \tilde{C}_{y}-y \tilde{C}_{x}}{\Phi_{\odot}} d z
$$

satisfies

$$
\begin{aligned}
d \beta_{2}= & -\frac{\partial_{x} C_{x}+\partial_{y} C_{y}+\partial_{z} C_{z}+\left(3-q_{1}(s)\right) x y z}{\Phi_{\ominus}^{2}} \Omega \\
& +\frac{\partial_{x} \tilde{C}_{x}+\partial_{y} \tilde{C}_{y}+\partial_{z} \tilde{C}_{z}}{\Phi_{\odot}} \Omega .
\end{aligned}
$$

Finally choosing $q_{0}(s)$ such that

$$
q_{0}(s)=-1+q_{1}(s)+\partial_{x} \tilde{C}_{x}+\partial_{y} \tilde{C}_{y}+\partial_{z} \tilde{C}_{z}
$$

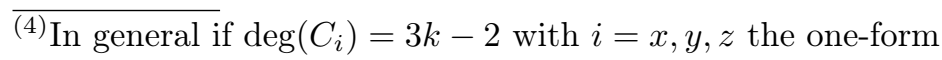

$$
\beta=\frac{y C_{z}-z C_{y}}{\Phi_{\ominus}^{k}} d x+\frac{z C_{x}-x C_{z}}{\Phi_{\ominus}^{k}} d y+\frac{x C_{y}-y C_{x}}{\Phi_{\odot}^{k}} d z
$$

satisfies

$$
d \beta=-k \frac{\left(C_{x} \partial_{x}+C_{y} \partial_{y}+C_{z} \partial_{z}\right) \Phi_{\ominus}}{\Phi_{\ominus}^{k+1}} \Omega+\frac{\partial_{x} C_{x}+\partial_{y} C_{y}+\partial_{z} C_{z}}{\Phi_{\ominus}^{k}} \Omega
$$


leads to

$$
L_{\ominus} \Omega_{\ominus}(s)=d\left(\beta_{1}+\beta_{2}\right) .
$$

The expression for $q_{0}(s)$ is (see (3.9) for the definitions of $M_{2}, M_{4}$ and $M_{6}$ )

$$
\begin{aligned}
q_{0}(s)= & -\frac{n_{0}}{\left(s^{2} \prod_{i=1}^{4} \mu_{i}-2 s M_{2}+3\right) \prod_{i=1}^{4}\left(\mu_{i}^{2} s-1\right)} \\
n_{0}= & -\mu_{1}^{3} \mu_{2}^{3} \mu_{3}^{3} \mu_{4}^{3} s^{6} \\
& +s^{5} \mu_{1} \mu_{2} \mu_{3} \mu_{4}\left(-3 M_{2}^{3}+12 M_{2} M_{4}+12 M_{6}\right) \\
& +s^{4}\left(-18 M_{2}^{4}+108 M_{2}^{2} M_{4}-120 M_{2} M_{6}-144 M_{4}^{2}\right) \\
& +s^{3}\left(26 M_{2}^{3}-96 M_{2} M_{4}+324 M_{6}\right) \\
& +s^{2}\left(24 M_{4}-15 M_{2}^{2}\right)+3 M_{2} s .
\end{aligned}
$$

Acting with the Picard-Fuchs operator on the sunset integral gives

$$
S_{\odot}(s)=\int_{\Delta} L_{\ominus} \Omega_{\odot}=\int_{\Delta} d \beta
$$

with $\beta=\beta_{1}+\beta_{2}=\beta_{x} d x+\beta_{y} d y+\beta_{z} d z$.

For evaluating this integral we consider the blow-up $\tilde{\Delta}$ of the domain of integration $\Delta=\left\{[x: y: z] \in \mathbb{P}^{2} \mid x, y, z \geq 0\right\}$, by putting a sphere of radius $\epsilon>0$ around each of the points $[1: 0: 0],[0: 1: 0]$ and $[0: 0: 1]$.

Integration by part gives the boundary contributions

$$
\begin{aligned}
S_{\ominus}(s)= & \lim _{\epsilon \rightarrow 0} \int_{\left.\partial \tilde{\Delta}\right|_{x=0}}\left(\beta_{y} d y+\beta_{z} d z\right) \\
& +\lim _{\epsilon \rightarrow 0} \int_{\left.\partial \tilde{\Delta}\right|_{y=0}}\left(\beta_{x} d x+\beta_{z} d z\right) \\
& +\lim _{\epsilon \rightarrow 0} \int_{\left.\partial \tilde{\Delta}\right|_{z=0}}\left(\beta_{x} d x+\beta_{y} d y\right) .
\end{aligned}
$$

Where $\left.\partial \tilde{\Delta}\right|_{x=0}$ denote the boundary of the blown-up domain $\tilde{\Delta}$ restricted to the plane $x=0$. Setting $\zeta=y / z$ in the first integral, setting $\zeta=z / x$ in the second integral and $\zeta=x / y$ in the last integral we obtain

$$
S_{\ominus}(s)=\lim _{\epsilon \rightarrow 0} \int_{\epsilon}^{\frac{1}{\epsilon}}\left(z \beta_{y}+x \beta_{z}+y \beta_{x}\right) d \zeta .
$$


With $z \beta_{y}=\left(a_{1}+b_{1} \zeta\right) /\left(\zeta\left(\xi_{3}^{2}+\xi_{2}^{2} \zeta\right)\right)$ and $y \beta_{x}=-\left(b_{1}+b_{2} \zeta\right) /\left(\zeta\left(\xi_{2}^{2}+\xi_{1}^{2} \zeta\right)\right)$ and $x \beta_{z}=$ $\left(-\xi_{1}^{2} b_{2} / 2+b_{3} \zeta-\xi_{3}^{2} a_{1} / 2 \zeta^{2}\right) /\left(\zeta\left(\xi_{1}^{2}+\xi_{3}^{2} \zeta\right)^{2}\right)$ where $a_{1}, b_{1}, b_{2}, b_{3}$ are polynomials in $s$ reading

$$
\begin{aligned}
a_{1}= & 4\left(\xi_{1}^{2}-\xi_{2}^{2}\right) \xi_{3}^{2} s\left(3-3 s\left(3 \xi_{1}^{2}+3 \xi_{2}^{2}-7 \xi_{3}^{2}\right)\right. \\
& +s^{2}\left(9 \xi_{1}^{4}-10 \xi_{1}^{2} \xi_{2}^{2}-14 \xi_{1}^{2} \xi_{3}^{2}+9 \xi_{2}^{4}-14 \xi_{2}^{2} \xi_{3}^{2}+5 \xi_{3}^{4}\right) \\
& \left.-3 s^{3} \mu_{1} \mu_{2} \mu_{3} \mu_{4}\left(\xi_{1}^{2}+\xi_{2}^{2}-\xi_{3}^{2}\right)\right)
\end{aligned}
$$

and $b_{1}$ is obtained from $a_{1}$ by exchanging $\xi_{2}$ and $\xi_{3}$, the coefficient $b_{2}$ is obtained from $a_{1}$ by exchanging $\xi_{1}$ and $\xi_{3}$, and finally

$$
\begin{aligned}
b_{3}= & 6 \xi_{1}^{2} \xi_{3}^{2}\left(9-s\left(13 \xi_{1}^{2}+10 \xi_{2}^{2}+13 \xi_{3}^{2}\right)\right. \\
& +s^{2}\left(\xi_{1}^{4}+27 \xi_{1}^{2} \xi_{2}^{2}+6 \xi_{1}^{2} \xi_{3}^{2}-8 \xi_{2}^{4}+27 \xi_{2}^{2} \xi_{3}^{2}+\xi_{3}^{4}\right) \\
& +s^{3}\left(\xi_{1}^{6}+4 \xi_{1}^{4} \xi_{2}^{2}-\xi_{1}^{4} \xi_{3}^{2}-15 \xi_{1}^{2} \xi_{2}^{4}-24 \xi_{1}^{2} \xi_{2}^{2} \xi_{3}^{2}\right. \\
& \left.\quad-\xi_{1}^{2} \xi_{3}^{4}+10 \xi_{2}^{6}-15 \xi_{2}^{4} \xi_{3}^{2}+4 \xi_{2}^{2} \xi_{3}^{4}+\xi_{3}^{6}\right) \\
& \left.+s^{4} \mu_{1} \mu_{2} \mu_{3} \mu_{4}\left(2 \xi_{1}^{4}-\xi_{1}^{2} \xi_{2}^{2}-4 \xi_{1}^{2} \xi_{3}^{2}-\xi_{2}^{4}-\xi_{2}^{2} \xi_{3}^{2}+2 \xi_{3}^{4}\right)\right) .
\end{aligned}
$$

The integral has a finite limit when $\epsilon \rightarrow 0$ given by

$$
S_{\ominus}(s)=\mathcal{Y}_{\odot}(s)-\frac{2 s \sum_{i=1}^{3} \log \left(\xi_{i}^{2}\right) c_{i}(s)}{\prod_{i=1}^{4}\left(s \mu_{i}^{2}-1\right)\left(s^{2} \prod_{i=1}^{4} \mu_{i}+2 s M_{2}-3\right)}
$$

where the Yukawa coupling is given by ${ }^{(5)}$

$$
\mathcal{Y}_{\ominus}(s)=2 \frac{s^{2} \prod_{i=1}^{4} \mu_{i}-2 s M_{2}+3}{\prod_{i=1}^{4}\left(\mu_{i}^{2} s-1\right)} .
$$

The coefficients satisfy $c_{1}(s)+c_{2}(u)+c_{3}(s)=0$ and are given by

$$
\begin{aligned}
c_{1}(s)= & -2 \xi_{1}^{2}+\xi_{2}^{2}+\xi_{3}^{2} \\
& +s\left(6 \xi_{1}^{4}-7 \xi_{1}^{2} \xi_{2}^{2}-3 \xi_{2}^{4}-7 \xi_{1}^{2} \xi_{3}^{2}+14 \xi_{2}^{2} \xi_{3}^{2}-3 \xi_{3}^{4}\right) \\
& +s^{2}\left(-6 \xi_{1}^{6}+11 \xi_{1}^{4} \xi_{2}^{2}-8 \xi_{1}^{2} x i_{2}^{4}+3 \xi_{2}^{6}+11 \xi_{1}^{4} \xi_{3}^{2}-3 \xi_{2}^{4} \xi_{3}^{2}-8 \xi_{1}^{2} \xi_{3}^{4}-3 \xi_{2}^{2} \xi_{3}^{4}+3 \xi_{3}^{6}\right) \\
& \quad-s^{3} \mu_{1} \mu_{2} \mu_{3} \mu_{4}\left(2 \xi_{1}^{4}-\xi_{1}^{2} \xi_{2}^{2}-\xi_{2}^{4}-\xi_{1}^{2} \xi_{3}^{2}+2 \xi_{2}^{2} \xi_{3}^{2}-\xi_{3}^{4}\right)
\end{aligned}
$$

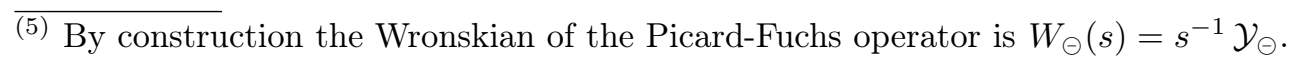


and $c_{2}(s)$ is obtained from $c_{1}(s)$ by exchanging $\xi_{1}$ and $\xi_{2}$

$$
\begin{aligned}
c_{2}(s)= & \xi_{1}^{2}-2 \xi_{2}^{2}+\xi_{3}^{2} \\
& +s\left(6 \xi_{2}^{4}-7 \xi_{1}^{2} \xi_{2}^{2}-3 \xi_{1}^{4}-7 \xi_{2}^{2} \xi_{3}^{2}+14 \xi_{1}^{2} \xi_{3}^{2}-3 \xi_{3}^{4}\right) \\
& +s^{2}\left(-6 \xi_{2}^{6}+11 \xi_{2}^{4} \xi_{1}^{2}-8 \xi_{2}^{2} x i_{1}^{4}+3 \xi_{1}^{6}+11 \xi_{2}^{4} \xi_{3}^{2}-3 \xi_{1}^{4} \xi_{3}^{2}-8 \xi_{2}^{2} \xi_{3}^{4}-3 \xi_{1}^{2} \xi_{3}^{4}+3 \xi_{3}^{6}\right) \\
& \quad-s^{3} \mu_{1} \mu_{2} \mu_{3} \mu_{4}\left(2 \xi_{2}^{4}-\xi_{1}^{2} \xi_{2}^{2}-\xi_{1}^{4}-\xi_{2}^{2} \xi_{3}^{2}+2 \xi_{1}^{2} \xi_{3}^{2}-\xi_{3}^{4}\right) .
\end{aligned}
$$

Remark 3.1. - In the all equal masses case $\xi_{1}=\xi_{2}=\xi_{3}=1$ we immediately have that $y \beta_{z}=0$ and $y \beta_{x}=0$ and

$$
x \beta_{y}=\frac{36}{(s-1)(9 s-1)(1+\zeta)^{2}}
$$

leading to $S_{\ominus}(s)=6 /((9 s-1)(s-1))$ which is the Yukawa coupling $\mathcal{Y}_{\odot}(s)$. The Picard-Fuchs operator reads (with $\delta_{s}:=s d / d s$ )

$$
L_{\ominus}=\delta_{s}^{2}+\frac{2 s(9 s-5)}{(s-1)(9 s-1)} \delta_{s}+\frac{3 s(3 s-1)}{(s-1)(9 s-1)} .
$$

The sunset integral satisfies the differential equation

$$
L_{\ominus}\left(-\frac{1}{s} \mathcal{I}_{\ominus}(s)\right)=\frac{6}{(9 s-1)(s-1)},
$$

which is equivalent to

$$
s(9 s-1)(s-1) \frac{d^{2} \mathcal{I}_{\ominus}(s)}{d s^{2}}+\left(9 s^{2}-1\right) \frac{d \mathcal{I}_{\ominus}(s)}{d s}+\frac{1-3 s}{s} \mathcal{I}_{\ominus}(s)=-6 .
$$

This differential equation has been presented in the following matrix form in [Hennb, eq. (4.13)]

$$
\frac{d}{d s} \vec{f}(s)=\frac{A_{0}}{s}+\frac{A_{1}}{s-1}+\frac{A_{9}}{9 s-1} .
$$

The poles are located at the singular fibers of the sunset elliptic curves family. The residues are the monodromy matrices $A_{0}, A_{1}$ and $A_{9}$ which are independent of $s$. These squared matrices have size three which equal the (generic) rank of the all equal masses sunset motive [BV]. This first order equation arises from the flat Gauß-Manin connection for the coherent analytic sheaf for which the section $\tilde{\sigma}$ leads to sunset Feynman integral according (4.2) as proven in lemma 6.21 of [BV] for the all equal masses case.

3.3. The elliptic dilogarithm. - For $s \in]\left(\xi_{1}+\xi_{2}+\xi_{3}\right)^{-2},+\infty[$ we provide an expression of the sunset integral $\mathcal{I}_{\ominus}(s)$ in (3.1) in term of the elliptic dilogarithms. A derivation via motives will given in section 4 . 
We start by considering the ratio of the coordinates on the sunset cubic curve as functions on $\mathbb{C}^{\times} / q^{\mathbb{Z}}$

$$
\begin{aligned}
\frac{X}{Z}(x) & =\frac{\theta_{1}\left(x / x\left(Q_{1}\right)\right) \theta_{1}\left(x / x\left(P_{3}\right)\right)}{\theta_{1}\left(x / x\left(P_{1}\right)\right) \theta_{1}\left(x / x\left(Q_{3}\right)\right)} \\
\frac{Y}{Z}(x) & =\frac{\theta_{1}\left(x / x\left(Q_{2}\right)\right) \theta_{1}\left(x / x\left(P_{3}\right)\right)}{\theta_{1}\left(x / x\left(P_{2}\right)\right) \theta_{1}\left(x / x\left(Q_{3}\right)\right)} .
\end{aligned}
$$

where $x(P)$ is the representation of the point $P$ in $\mathcal{E}_{\ominus} \simeq \mathbb{C}^{\times} / q^{\mathbb{Z}}$ using the map of section 3.1.1, and $\theta_{1}(x)$ is the Jacobi theta function

$$
\theta_{1}(x)=q^{\frac{1}{8}} \frac{x^{1 / 2}-x^{-1 / 2}}{\mathbf{i}} \prod_{n \geq 1}\left(1-q^{n}\right)\left(1-q^{n} x\right)\left(1-q^{n} / x\right) .
$$

We evaluate the integral

$$
F(x)=-\int_{x_{0}}^{x} \log \left(\frac{X}{Z}(y)\right) d \log y
$$

where $x_{0}$ is an arbitrary origin that will cancel in the final answer. We find

$$
\begin{aligned}
F(x)= & F\left(x_{0}\right)+E_{2}\left(x / x\left(P_{1}\right)\right)+E_{2}\left(x / x\left(Q_{3}\right)\right) \\
& -E_{2}\left(x / x\left(P_{3}\right)\right)-E_{2}\left(x / x\left(Q_{1}\right)\right)
\end{aligned}
$$

where $E_{2}(x)$ is the elliptic dilogarithm

$$
E_{2}(x)=\sum_{n \geq 0} \operatorname{Li}_{2}\left(q^{n} x\right)-\sum_{n \geq 1} \operatorname{Li}_{2}\left(q^{n} / x\right)+\frac{1}{4}(\log (x))^{2}-\mathbf{i} \pi \log (x)
$$

Using the 2-torsion relations $x\left(Q_{i}\right)=-x\left(P_{i}\right)$ for $i=1,2,3$ we can rewrite $F(x)$ as

$$
F(x)=\hat{E}_{2}\left(x / x\left(P_{1}\right)\right)-\hat{E}_{2}\left(x / x\left(P_{3}\right)\right)+\frac{\mathbf{i} \pi}{2} \log \left(\frac{x\left(P_{1}\right)}{x\left(P_{3}\right)}\right)+F\left(x_{0}\right),
$$

where

$$
\begin{aligned}
\hat{E}_{2}(x)= & \sum_{n \geq 0}\left(\operatorname{Li}_{2}\left(q^{n} x\right)-\operatorname{Li}_{2}\left(-q^{n} x\right)\right) \\
& -\sum_{n \geq 1}\left(\operatorname{Li}_{2}\left(q^{n} / x\right)-\operatorname{Li}_{2}\left(-q^{n} / x\right)\right) .
\end{aligned}
$$

With this we can evaluate on the zero or poles of $Y / Z$

$$
\mathcal{L}_{2}\left\{\frac{X}{Z}, \frac{Y}{Z}\right\}=F\left(x\left(P_{3}\right)\right)+F\left(x\left(Q_{2}\right)\right)-F\left(x\left(P_{2}\right)\right)-F\left(x\left(Q_{3}\right)\right) .
$$


The origin of the integral $F\left(x_{0}\right)$ has cancelled in the expression. Using the expression for $F(x)$ in $(3.63)$ one gets

$$
\begin{aligned}
\mathcal{L}_{2}\left\{\frac{X}{Z}, \frac{Y}{Z}\right\}= & \hat{E}_{2}\left(\frac{-x\left(P_{2}\right)}{x\left(P_{1}\right)}\right)-\hat{E}_{2}\left(\frac{x\left(P_{2}\right)}{x\left(P_{1}\right)}\right)+\hat{E}_{2}\left(\frac{x\left(P_{2}\right)}{x\left(P_{3}\right)}\right) \\
& -\hat{E}_{2}\left(\frac{-x\left(P_{2}\right)}{x\left(P_{3}\right)}\right)+\hat{E}_{2}\left(\frac{x\left(P_{3}\right)}{x\left(P_{1}\right)}\right)-\hat{E}_{2}\left(\frac{-x\left(P_{3}\right)}{x\left(P_{1}\right)}\right) \\
& +\hat{E}_{2}(-1)-\hat{E}_{2}(1) .
\end{aligned}
$$

Noticing the following properties of the function $\hat{E}_{2}(x)$

$$
\begin{aligned}
& \hat{E}_{2}(-x)=-\hat{E}_{2}(x) \\
& \hat{E}_{2}(1 / x)=-\hat{E}_{2}(x)+\mathrm{Li}_{2}(x)-\mathrm{Li}_{2}(-x)+\mathrm{Li}_{2}\left(\frac{1}{x}\right)-\mathrm{Li}_{2}\left(-\frac{1}{x}\right)
\end{aligned}
$$

together with the dilogarithm functional equation

$$
\mathrm{Li}_{2}(x)+\mathrm{Li}_{2}(1 / x)=-\frac{\pi^{2}}{6}-\frac{1}{2} \log (-x)^{2}
$$

we can reduce the expression for $\mathcal{L}_{2}\{X / Z, Y / Z\}$ to

$$
\begin{aligned}
\mathcal{L}_{2}\left\{\frac{X}{Z}, \frac{Y}{Z}\right\}= & 2 \hat{E}_{2}\left(\frac{x\left(P_{1}\right)}{x\left(P_{2}\right)}\right)+2 \hat{E}_{2}\left(\frac{x\left(P_{2}\right)}{x\left(P_{3}\right)}\right)+2 \hat{E}_{2}\left(\frac{x\left(P_{3}\right)}{x\left(P_{1}\right)}\right) \\
& +\frac{\pi^{2}}{4}-\mathbf{i} \pi \log \left(\frac{x\left(P_{1}\right)}{x\left(P_{2}\right)}\right) .
\end{aligned}
$$

The elliptic dilogarithm $\hat{E}_{2}(x)$ is not invariant under $q$-translation and transforms according

$$
\begin{gathered}
\hat{E}_{2}(q x)=\hat{E}_{2}(x)-\frac{\pi^{2}}{2}+\mathbf{i} \pi \log (x) \\
\hat{E}_{2}(x / q)=\hat{E}_{2}(x)+\frac{\pi^{2}}{2}-\mathbf{i} \pi \log (x / q) .
\end{gathered}
$$

This is because the Feynman integral we are studying is a multivalued function. Shifting the representative $x(P)$ of the point $P$ in $\mathbb{C}^{\times} / q^{\mathbb{Z}}$ changes the expression for $\mathcal{L}_{2}\left\{\frac{X}{Z}, \frac{Y}{Z}\right\}$ modulo $\mathbf{i} \pi \log q, \mathbf{i} \pi \log \left(x\left(P_{1}\right)\right), \mathbf{i} \pi \log \left(x\left(P_{2}\right)\right)$ or $\mathbf{i} \pi \log \left(x\left(P_{3}\right)\right)$.

In order to fix this ambiguity we symmetrize the computation by summing other all the other choices to get

$$
\begin{aligned}
\mathcal{L}_{2} & :=\mathcal{L}_{2}\left\{\frac{X}{Z}, \frac{Y}{Z}\right\}+\mathcal{L}_{2}\left\{\frac{X}{Y}, \frac{Z}{Y}\right\}+\mathcal{L}_{2}\left\{\frac{Y}{X}, \frac{Z}{X}\right\} \\
& =6 \hat{E}_{2}\left(\frac{x\left(P_{1}\right)}{x\left(P_{2}\right)}\right)+6 \hat{E}_{2}\left(\frac{x\left(P_{2}\right)}{x\left(P_{3}\right)}\right)+6 \hat{E}_{2}\left(\frac{x\left(P_{3}\right)}{x\left(P_{1}\right)}\right)+\frac{3 \pi^{2}}{4} .
\end{aligned}
$$


3.3.1. The all equal masses case. - It was shown in $[\mathrm{BV}]$ that the all equal masses case $\xi_{1}=\xi_{2}=\xi_{3}=1$ sunset integral is given by

$$
\mathcal{I}_{\ominus}\left(s_{\ominus}(q)\right)=\frac{\varpi_{r}}{\pi}\left(\mathbf{i} \pi^{2}(1-2 \tau)+E_{\ominus}(q)\right)
$$

where $q=\exp (2 \pi \mathbf{i} \tau)$ with $\tau=\varpi_{c} / \varpi_{r}$ the period ratio and $s_{\ominus}$ is the Hauptmodul

$$
s_{\ominus}(q)^{-1}=9+72 \frac{\eta\left(q^{2}\right)}{\eta\left(q^{3}\right)}\left(\frac{\eta\left(q^{6}\right)}{\eta(q)}\right)^{5}
$$

and $E_{\ominus}(q)$ is the elliptic dilogarithm evaluated that the sixth root of unity $\zeta_{6}=e^{\frac{\mathbf{i} \pi}{3}}$

$$
\begin{aligned}
E_{\ominus}(q)= & \frac{1}{2 \mathbf{i}} \sum_{n \geq 1}\left(\operatorname{Li}_{2}\left(q^{n} \zeta_{6}\right)+\operatorname{Li}_{2}\left(q^{n} \zeta_{6}^{2}\right)-L i_{2}\left(q^{n} \zeta_{6}^{4}\right)-\operatorname{Li}_{2}\left(q^{n} \zeta_{6}^{5}\right)\right) \\
& +\frac{1}{4 \mathbf{i}}\left(\operatorname{Li}_{2}\left(\zeta_{6}\right)+L i_{2}\left(\zeta_{6}^{2}\right)-\operatorname{Li}_{2}\left(\zeta_{6}^{4}\right)-\operatorname{Li}_{2}\left(\zeta_{6}^{5}\right)\right) .
\end{aligned}
$$

Noticing that

$$
2 \mathbf{i} E_{\ominus}(q)=\hat{E}_{2}\left(\zeta_{6}^{2}\right)+\zeta(2)
$$

and since when all the masses are equal the image in $\mathbb{C}^{\times} / q^{\mathbb{Z}}$ of the points $x\left(P_{i}\right)=\zeta_{6}^{i}$ with $i=1,2,3$, we have

$$
\mathcal{L}_{2}\left\{\frac{X}{Z}, \frac{Y}{Z}\right\}=2 \mathbf{i} E_{\ominus}(q)+\frac{11 \pi^{2}}{3}
$$

Showing that the all equal masses sunset integral is equal to the regulator (3.77) modulo periods of the elliptic curves

$$
I_{\ominus} \equiv \frac{\varpi_{r}}{2 \pi \mathbf{i}} \mathcal{L}_{2}\left\{\frac{X}{Z}, \frac{Y}{Z}\right\} \quad \bmod \text { periods } .
$$

3.3.2. Three masses case. - In the three masses case the sunset integral in (3.1) is given by

$$
\mathcal{I}_{\ominus}(s) \equiv \frac{\mathbf{i} \varpi_{r}}{\pi}\left(\hat{E}_{2}\left(\frac{x\left(P_{1}\right)}{x\left(P_{2}\right)}\right)+\hat{E}_{2}\left(\frac{x\left(P_{2}\right)}{x\left(P_{3}\right)}\right)+\hat{E}_{2}\left(\frac{x\left(P_{3}\right)}{x\left(P_{1}\right)}\right)\right) \quad \bmod \text { periods }
$$

An expression in terms of multiple polylogarithms has been presented in $[\mathrm{ABW} 2$, $\mathrm{ABW}$ 3, $\mathrm{ABW}$ 4, $\mathrm{ABW}$ ]. It would be interesting to relate these results.

A proof is given in section 4 using a motivic approach. In this section we present numerical verification of this expression for the sunset integral.

According (3.70) the elliptic dilogarithm $\hat{E}_{2}(x)$ is not invariant under the change $x\left(P_{i}\right) \rightarrow q x\left(P_{i}\right)$ therefore the expression in (3.79) shifts by $\mathbf{i} \pi \log q$. Therefore by changing the representative of $P_{1}, P_{2}$ and $P_{3}$ in $\mathbb{C}^{\times} / q^{\mathbb{Z}}$ one can change the coefficients of the periods of the elliptic curve freely. 
3.3.3. Numerical checks. - We have made some numerical checks (see table 1 on page 19) of this relation using PARI/GP [Pari]. For given values of the masses and $s$ we have searched for an integer linear dependence of the vector

$$
v=\left[\mathcal{I}_{\ominus}(s)-\mathbf{i} \frac{\varpi_{r}}{\pi} \mathcal{L}_{2}\left\{\frac{X}{Z}, \frac{Y}{Z}\right\}, \mathbf{i} \pi \varpi_{r}, \mathbf{i} \pi \varpi_{c}\right]
$$

using the lindep command of PARI/GP. The vector is composed of the sunset integral evaluated using the Bessel integral representation [GKPa, BBDG, GKPb, Va]

$$
\mathcal{I}_{\ominus}(s)=\int_{0}^{\infty} 4 x I_{0}\left(\sqrt{s^{-1}} x\right) \prod_{i=1}^{3} K_{0}\left(\xi_{i} x\right) d x
$$

the regulator evaluated as

$$
\mathcal{L}_{2}\left\{\frac{X}{Z}, \frac{Y}{Z}\right\}=\hat{E}_{2}\left(x_{12}\right)+\hat{E}_{2}\left(x_{23}\right)+\hat{E}_{2}\left(x_{31}\right)
$$

where $x_{i j}=x\left(P_{i}\right) / x\left(P_{j}\right)$ in $\mathbb{C}^{\times} / q^{\mathbb{Z}}$. Since we can easily follow the change of the expression under a $q$-translation of the points, we have made some choices such that the relation between the sunset integral and the regulator is modulo periods of the elliptic curves with simple rational coefficients, keeping the relation $x_{12} x_{23} x_{31}=1$. For instance in table 1 for the case $\left(\xi_{1}, \xi_{2}, \xi_{3}, s^{-1}\right)=(1,2,3,3)$, we show how the $q$ translations $\left(x_{12}, x_{31}\right) \rightarrow\left(q x_{12}, x_{31} / q\right)$ affect the result modulo periods of the elliptic curve.

\begin{tabular}{||c|c|c|c|c|c||}
\hline$\xi_{1}, \xi_{2}, \xi_{3}, s^{-1}$ & $x_{21}$ & $x_{32}$ & $q$ & lindep (v) & prec \\
\hline $1,2,8,2$ & $-0.00931124+0.0160094 \mathbf{i}$ & $4.87147-5.50124 \mathbf{i}$ & 0.136089 & {$[4,3,-8]$} & $210^{-36}$ \\
$1,2,8,6$ & $-0.00640431+0.00671999 \mathbf{i}$ & $6.17736-8.34052 \mathbf{i}$ & 0.0963482 & {$[4,3,-8]$} & $210^{-36}$ \\
& & & & & \\
$1,2,3,2$ & $0.0733690-0.108597 \mathbf{i}$ & $0.797236-0.603668 \mathbf{i}$ & -0.131059 & {$[4,1,0]$} & $610^{-37}$ \\
$1,2,3,2$ & $-0.00961565+0.0142326 \mathbf{i}$ & $-6.08304+4.60608 \mathbf{i}$ & -0.131059 & {$[4,-5,8]$} & $610^{-37}$ \\
$1,2,3,3$ & $-0.723282-0.690553 \mathbf{i}$ & $-0.145143-0.107284 \mathbf{i}$ & -0.180489 & {$[4,3,-8]$} & $510^{-37}$ \\
& & & & & \\
$1,5,7,3$ & $-0.481821+0.876270 \mathbf{i}$ & $-0.0416592+0.0163910 \mathbf{i}$ & -0.0447678 & {$[4,-5,8]$} & $10^{-37}$ \\
$1,5,7,7$ & $-0.766655+0.642059 \mathbf{i}$ & $-7.08429+2.58610 \mathbf{i}$ & -0.132599 & {$[4,-5,8]$} & $710^{-38}$ \\
& & & & & \\
$3,5,7,3$ & $0.199999+0.979796 \mathbf{i}$ & $-6.29720+3.35123 \mathbf{i}$ & -0.140185 & {$[4,-5,8]$} & $710^{-38}$ \\
$3,5,7,7$ & $-0.199528+0.979892 \mathbf{i}$ & $-5.76891+4.08260 \mathbf{i}$ & -0.141495 & {$[4,-5,8]$} & $510^{-38}$ \\
\hline
\end{tabular}

TABLE 1. Results of linear dependence of vector $v$ defined in (3.80) using the PARI/GP command lindep(v). The last column gives the absolute value for the numerical evaluation linear relations. 


\section{Approach via Motives}

The purpose of this section is to prove formula (3.79) for the sunset Feynman integral in two dimensions with arbitrary masses. This is a beautiful illustration how the theory of motives can yield information about Feynman integrals. With an eye toward future applications, we will permit ourselves to say a bit more than what is strictly necessary for the sunset case.

We fix masses and external momenta and just write $E$ for the resulting elliptic curve, which is an element in the family (3.5). We have $E \hookrightarrow \mathbb{P}^{2}$ with homogeneous coordinates $X, Y, Z$, and $E$ meets the coordinate triangle $X Y Z=0$ in a set $S$ of 6 points, $S:=\left\{P_{1}, P_{2}, P_{3}, Q_{1}, Q_{2}, Q_{3}\right\}$. Let $E^{0}:=E-S$. Following [BV, §6], let $\rho: P \rightarrow \mathbb{P}^{2}$ be the blowup of the vertices of the coordinate triangle, and let $\mathfrak{h} \subset P$ be the resulting hexagon. We can lift $E \hookrightarrow P$ and write $\mathfrak{h}^{0}=\mathfrak{h}-E \cap \mathfrak{h}$.

Let $\sigma \subset \mathbb{P}^{2}(\mathbb{R})$ be the positive real simplex which is the chain of integration for the Feynman integral. for general values of external momenta, $\sigma$ will not meet $E$, and we can lift to $\widetilde{\sigma} \subset P-E$. We have $\partial \widetilde{\sigma} \subset \mathfrak{h}^{0}$, so

$$
\widetilde{\sigma} \in H_{2}\left(P-E, \mathfrak{h}^{0} ; \mathbb{Q}\right)=H^{2}\left(P-E, \mathfrak{h}^{0} ; \mathbb{Q}\right)^{\vee} .
$$

The form $\Omega_{\ominus},(3.3)$, represents a class in $F^{2} H^{2}\left(P-E, \mathfrak{h}^{0} ; \mathbb{C}\right)$, and the Feynman integral

$$
\mathcal{I}_{\ominus}=\left\langle\Omega_{\ominus}, \tilde{\sigma}\right\rangle \text {. }
$$

The idea is to interpret $\mathcal{I}_{\ominus}$ as a quantity intrinsic to the Hodge structure $H^{2}(P-$ $\left.E, \mathfrak{h}^{0} ; \mathbb{Q}\right)$ together with the choice of $\Omega_{\ominus}$. That way, whenever we see the Hodge structure (and we will see it in two other guises below) we can be sure that the sunset Feynman integral $\mathcal{I}_{\ominus}$ is involved.

To begin, we can invoke $[\mathrm{BKV}]$, lemma 6.1.4 to get

$$
H_{2}\left(P-E, \mathfrak{h}^{0} ; \mathbb{Q}\right)=H^{2}\left(P-E, \mathfrak{h}^{0} ; \mathbb{Q}\right)^{\vee} \cong H^{2}\left(\mathbb{G}_{m}^{2}, E^{0} ; \mathbb{Q}(2)\right)
$$

Here we identify

$$
\mathbb{G}_{m}^{2}=\mathbb{P}^{2}-\{X Y Z=0\}=P-\mathfrak{h} .
$$

We consider the long-exact sequence of Hodge structures

$$
\begin{aligned}
H^{1}\left(\mathbb{G}_{m}^{2}, \mathbb{Q}(2)\right) \stackrel{\alpha}{\rightarrow} H^{1}\left(E^{0}, \mathbb{Q}(2)\right) & \rightarrow H^{2}\left(\mathbb{G}_{m}^{2}, E^{0} ; \mathbb{Q}(2)\right) \\
& \rightarrow H^{2}\left(\mathbb{G}_{m}^{2}, \mathbb{Q}(2)\right) \rightarrow 0 .
\end{aligned}
$$

The image of $\alpha$ above is spanned by the logarithmic classes

$$
d \log (X / Z), d \log (Y / Z) .
$$

We can avoid these by replacing $\mathbb{G}_{m}^{2}$ by the relative space $\left(\mathbb{G}_{m},\{1\}\right)^{2}$. One has

$$
H^{i}\left(\left(\mathbb{G}_{m},\{1\}\right)^{n}, \mathbb{Q}\right)= \begin{cases}0 & i \neq n \\ \mathbb{Q}(-n) & i=n\end{cases}
$$


We now build a diagram

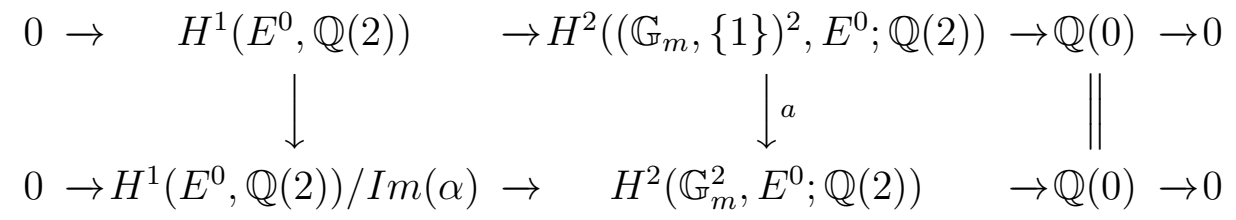

where the bottom line comes from truncating (4.5).

We are interested in the extensions of Hodge structures associated to these sequences. Since the sequence on the bottom comes by pushout, it will suffice to consider the top line. We consider splittings $s_{\mathbb{Q}} \in$ $H^{2}\left(\left(\mathbb{G}_{m},\{1\}\right)^{2}, E^{0} ; \mathbb{Q}(2)\right)$ and $s_{F} \in F^{0} H^{2}\left(\left(\mathbb{G}_{m},\{1\}\right)^{2}, E^{0} ; \mathbb{C}(2)\right)$ lifting $1 \in \mathbb{Q}(0)$. The obstruction to splitting the sequence of Hodge structures (4.7) is

$$
s_{\mathbb{Q}}-s_{F} \in H^{1}\left(E^{0}, \mathbb{C}(2)\right) / H^{1}\left(E^{0}, \mathbb{Q}(2)\right)
$$

We can choose $s_{\mathbb{Q}}$ so its image in $H^{2}\left(\mathbb{G}_{m}^{2}, E^{0} ; \mathbb{Q}(2)\right)$ coincides with $\widetilde{\sigma}$ under the identification (4.3). Indeed, the boundary $\partial \widetilde{\sigma}=1 \in H_{1}\left(\mathfrak{h}^{0}\right) \cong \mathbb{Q}(0)$. Also, in (4.7) the dual to the map labeled $a$ induces an isomorphism on $F^{2}$. (This is because $\operatorname{Im}(\alpha)^{\vee}=\mathbb{Q}(-1)^{2}$ and $F^{2} \mathbb{C}(-1)=(0)$.) In particular, $\Omega_{\ominus}$ lifts canonically to an element

$$
\Omega \in F^{2} H^{2}\left(\left(\mathbb{G}_{m},\{1\}\right)^{2}, E^{0} ; \mathbb{C}(2)\right)^{\vee} .
$$

Note that this element is orthogonal to $F^{-1} H^{2}\left(\left(\mathbb{G}_{m},\{1\}\right)^{2}, E^{0} ; \mathbb{C}(2)\right)$ so in particular it kills $s_{F} \in F^{0} \subset F^{-1}$. We conclude

$$
\mathcal{I}_{\ominus}=\left\langle\Omega_{\ominus}, \tilde{\sigma}\right\rangle=\left\langle\Omega, s_{\mathbb{Q}}-s_{F}\right\rangle .
$$

Our objective now is to reinterpret $I_{\odot}$ in terms of elliptic dilogarithms. Let $\square:=$ $\mathbb{P}^{1}-\{1\}$ and write $\partial \square=\{0, \infty\}$. Poincaré duality yields an identification

$$
H^{*}\left((\square, \partial \square)^{n}\right) \cong H^{2 n-*}\left(\left(\mathbb{G}_{m}, 1\right)^{n}\right)(n)^{\vee} \cong \begin{cases}\mathbb{Z}(0) & *=n \\ 0 & \text { else. }\end{cases}
$$

Let $\Gamma^{0} \subset E^{0} \times \mathbb{G}_{m}^{2}$ be the graph of the embedding $E^{0} \hookrightarrow \mathbb{G}_{m}^{2}$. Note that $\Gamma^{0}$ is actually closed in $E^{0} \times\left(\mathbb{P}^{1}\right)^{2}$ and we may intersect to get a closed codimension 2 cycle which we also call $\Gamma^{0}$ on $X \times\left(\mathbb{P}^{1}-\{1\}\right)^{2}$. This cycle doesn't meet the loci where coordinates $\in\{0, \infty\}$. The Gysin sequence yields

$$
\begin{gathered}
0 \rightarrow H^{3}\left(E^{0} \times(\square, \partial \square)^{2}\right)(2) \rightarrow H^{3}\left(E^{0} \times(\square, \partial \square)^{2}-\Gamma^{0}\right)(2) \rightarrow \mathbb{Z}(0) \rightarrow 0 \\
\| \\
H^{1}\left(E^{0}\right)(2)
\end{gathered}
$$

Lemma 4.1. - The sequence (4.11) and the top row of (4.7) agree as extensions of Hodge structure. 
Proof. - Note that we can generalize the top row of (4.7) to an extension

$$
0 \rightarrow H^{n-1}(X)(n) \rightarrow H^{n}\left(\left(\mathbb{G}_{m},\{1\}\right)^{n}, X\right)(n) \rightarrow \mathbb{Q}(0) \rightarrow 0
$$

for any $f: X \rightarrow \mathbb{G}_{m}^{n}$. (To avoid technicalities, we assume in the sequel that $X$ is smooth). We will construct a commutative diagram

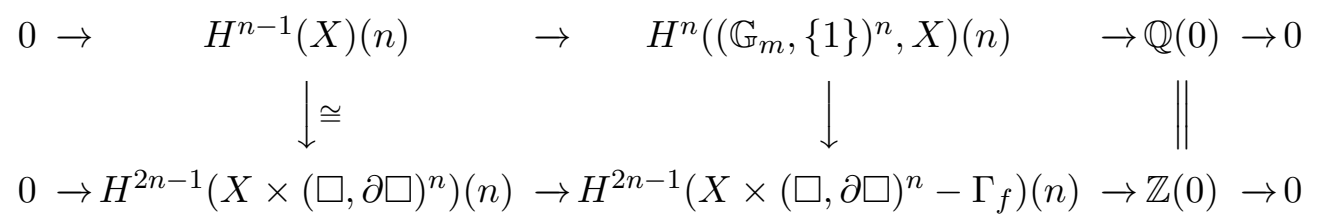

We consider the universal case $X=\mathbb{G}_{m}^{n}, f=i d$. Let $\Xi \subset \mathbb{G}_{m}^{n} \times \square^{n}$ be the corresponding graph. Note $\Xi \cong\left(\mathbb{G}_{m}-\{1\}\right)^{n}$. We want to understand $H^{*}\left(\left(\mathbb{G}_{m},\{1\}\right)^{n} \times\right.$ $\left.(\square, \partial \square)^{n}-\Xi\right)$. Consider the projection

$$
p:\left(\mathbb{G}_{m},\{1\}\right)^{n} \times(\square, \partial \square)^{n}-\Xi \rightarrow \mathbb{G}_{m}^{n} .
$$

The cohomology on the left is calculated by the sheaf $\mathcal{S}$ which is the constant sheaf with fibre $\mathbb{Q}$ on $\left(\mathbb{G}_{m}-\{1\}\right)^{n} \times(\square-\partial \square)^{n}$ extended by 0 to $\mathbb{G}_{m}^{n} \times \square^{n}$ and then restricted to the complement of $\Xi$. For $z=\left(z_{1}, \ldots, z_{n}\right) \in \mathbb{G}_{m}^{n}$ we have

$$
\left.\mathcal{S}\right|_{p^{-1}(z)}= \begin{cases}\mathbb{Q}_{\square^{n}-\{z\}} & \text { no } z_{i}=1 \\ (0) & \text { some } z_{i}=1\end{cases}
$$

The cohomology along the fibres of $p$ is thus

$$
H^{*}\left(\left.\mathcal{S}\right|_{p^{-1}(z)}\right)= \begin{cases}(0) & * \neq n, 2 n-1 ; \text { or some } z_{i}=1 \\ \mathbb{Z}(0) & *=n ; \text { no } z_{i}=1 \\ \mathbb{Z}(-n) & *=2 n-1 ; \text { no } z_{i}=1\end{cases}
$$

Using again that $H^{*}\left(\left(\mathbb{G}_{m},\{1\}\right)^{n}\right)=(0)$ for $* \neq n$, we conclude that in the Leray spectral sequence associated to $p,(4.14)$, one has

$$
E_{2}^{a b} \Rightarrow H^{a+b}\left(\left(\mathbb{G}_{m},\{1\}\right)^{n} \times(\square, \partial \square)^{n}-\Xi\right)
$$

and $E_{2}^{a b}=(0)$ unless $a=n$ and $b=n, 2 n-1$. In particular,

$$
H^{2 n-1}\left(\left(\mathbb{G}_{m},\{1\}\right)^{n} \times(\square, \partial \square)^{n}-\Xi\right)=(0) .
$$

The Gysin sequence yields

$$
\begin{aligned}
0 \rightarrow H^{0}(\Xi)(-n) & \stackrel{\text { gysin }}{\longrightarrow} H^{2 n}\left(\left(\mathbb{G}_{m},\{1\}\right)^{n} \times(\square, \partial \square)^{n}\right) \\
& \stackrel{\text { restrict }}{\longrightarrow} H^{2 n}\left(\left(\mathbb{G}_{m},\{1\}\right)^{n} \times(\square, \partial \square)^{n}-\Xi\right) .
\end{aligned}
$$

Both domain and target of the map labeled gysin are $\mathbb{Q}(-n)$. Since this map is injective, it is an isomorphism, so the map labeled restrict is zero. 
We now have a diagram (to shorten we write $\left.B=(\square, \partial \square)^{n}\right)$

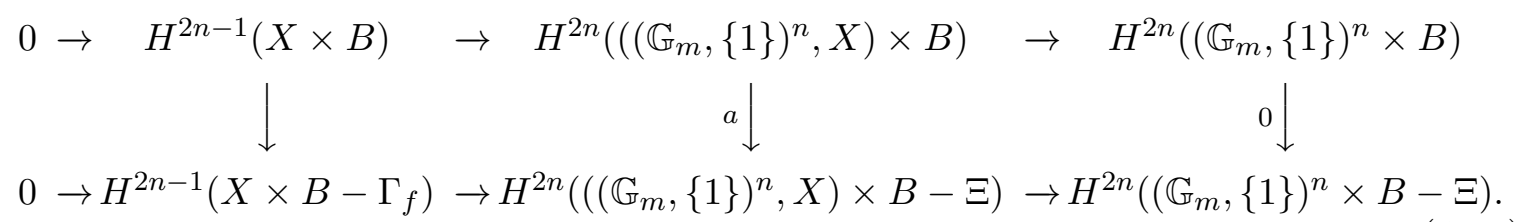

As a consequence of the above calculations, the map on the lower left is injective and the vertical map on the right is zero. It follows that the vertical map labeled $a$ lifts to $\tilde{a}$ fitting into a diagram

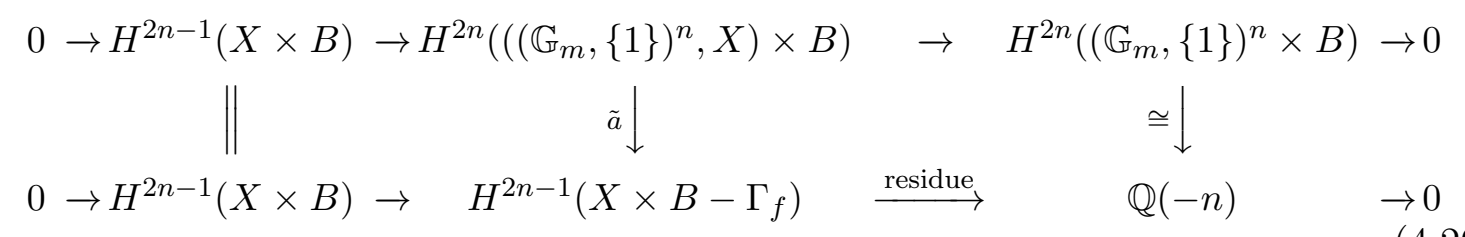

After twisting by $\mathbb{Z}(n)$ we find (4.20) coincides with (4.13), proving the lemma.

We can now compute $\mathcal{I}_{\odot}$ using (4.11). We have, again by lemma 6.1.4 in [BKV] (writing $S:=E-E^{0}$ )

$$
H^{3}\left(E^{0} \times(\square, \partial \square)^{2}\right)(2)^{\vee}=H^{3}\left((E, S) \times\left(\mathbb{G}_{m},\{1\}\right)^{2}\right)(1) .
$$

We fix coordinates $x, y$ on $\mathbb{G}_{m}^{2}$ and a holomorphic 1 -form de on $E$. The role of $\Omega$ in (4.9) will be played by

$$
\eta:=d e \wedge d x / x \wedge d y / y \in F^{2}\left(H^{3}\left((E, S) \times\left(\mathbb{G}_{m},\{1\}\right)^{2}\right)(1)\right) .
$$

Let $S:=E-E^{0}$. A homological interpretation of the top row of (4.7) is rather tricky. We need to define the group $H_{3}\left((E, S) \times\left(\mathbb{G}_{m},\{1\}\right)^{2}, \Gamma ; \mathbb{Q}\right)$ where $\Gamma \cong E$ is the complete curve. To justify this, let

$$
\mathbb{G}_{m}-\{1\} \stackrel{\ell}{\hookrightarrow} \mathbb{G}_{m} \stackrel{k}{\hookrightarrow} \mathbb{P}^{1} ; \quad E^{0} \stackrel{j}{\hookrightarrow} E
$$

be the open immersions. Let $f: E \hookrightarrow E \times \mathbb{P}^{1} \times \mathbb{P}^{1}$ extend the graph $E^{0} \hookrightarrow E^{0} \times \mathbb{G}_{m}^{2}$. The point is that the natural map over $E^{0}$ extends to

$$
f^{*}\left(j_{!} \mathbb{Q}_{E^{0}} \otimes k_{*} \ell_{!} \mathbb{Q}_{\mathbb{G}_{m}-\{1\}} \otimes k_{*} \ell_{!} \mathbb{Q}_{\mathbb{G}_{m}-\{1\}}\right) \rightarrow \mathbb{Q}_{E}
$$

This is because the points on $E \times \mathbb{P}^{1} \times \mathbb{P}^{1}$ where $\Gamma$ meets $\left(\{0, \infty\} \times \mathbb{P}^{1}\right) \cup\left(\mathbb{P}^{1} \times\{0, \infty\}\right)$ are contained in $S \times \mathbb{P}^{1} \times \mathbb{P}^{1}$ so the stalks of the sheaf $j_{!} \mathbb{Q}_{E^{0}} \bigotimes k_{*} \ell_{!} \mathbb{Q}_{\mathbb{G}_{m}-\{1\}} \bigotimes k_{*} \ell_{!} \mathbb{Q}_{\mathbb{G}_{m}-\{1\}}$ are zero.

We will integrate $\eta$ over a relative homology 3-chain $C$ on $E \times \mathbb{P}^{2}$. An argument (left to the reader) similar to the above will show that $C$ represents a class in $H_{3}((E, S) \times$ $\left.\left(\mathbb{G}_{m},\{1\}\right)^{2}, \Gamma ; \mathbb{Q}\right)$ and that $\partial C=\Gamma$. Define (cf. [KLM])

$$
C:=\{(e,(1-v)+v x(e), y(e)) \mid e \in E, 0 \leq v \leq 1\}
$$


Cut $E$ and $C$ along the locus $T_{y}:=\{e \mid y(e) \leq 0\}$. On the cut chain we can write $d y(e) / y(e)=d(\log y(e))$ and apply Stokes theorem. (More precisely, $T_{y}$ is an infinitely thin strip with two sides. The value of $\log y$ differs by $2 \pi \mathbf{i}$ at corresponding points on the two sides of $T_{y}$, so we find

$$
\int_{C} \eta=2 \pi \mathbf{i} \int_{\gamma} \log (x) d e
$$

where $\gamma$ is a 1 -chain with $\partial \gamma=(y)$, the divisor of $y=Y / Z$ on $E$. Using (4.9) and lemma 4.1, we deduce

Proposition 4.2. - The sunset Feynman integral

$$
\mathcal{I}_{\ominus}=\kappa \int_{\gamma} \log (x) \cdot \eta
$$

where $\kappa \eta=\Omega$ under the identification

$$
F^{3} H^{3}\left((E, S) \times\left(\mathbb{G}_{m},\{1\}\right)^{2}, \mathbb{C}\right)=F^{2} H^{3}\left(E \times\left(\mathbb{G}_{m},\{1\}\right)^{2}, \mathbb{C}(1)\right) .
$$

Remark 4.3. - Note that the 2-chain $\widetilde{\sigma}$ (4.1) defines (after reinterpretation in terms of cohomology as explained above) a splitting of the bottom row of (4.7). This chain does not lift to yield a splitting of the top row. This is because the chain $\sigma$ meets the lines $X=Z$ and $Y=Z$ in $\mathbb{P}^{2}$ and so does not represent a class in $H_{2}\left(\mathbb{P}^{2}-E-\right.$ $\{X=Z\}-\{Y=Z\})$. The effect of this is to introduce some elementary log terms corresponding to periods of $d(X / Z) /(X / Z)$ in (3.69).

Consider one last time the top sequence from (4.7). Writing $M$ for the middle group in this sequence, we see that the weight-graded pieces are

$$
W_{i} M_{\mathbb{Q}}= \begin{cases}H^{1}(E, \mathbb{Q}(2)) & i=-3 \\ \bigoplus_{5} \mathbb{Q}(1) & i=-2 \\ \mathbb{Q}(0) & i=0 .\end{cases}
$$

$M$ should be viewed as a representation of a sort of generalized graded Lie algebra with graded pieces the above pure Hodge structures (or pure motives). The Feynman integral is a period associated to the lower lefthand corner of the representation matrix. In trying to generalize to more complicated Feynman diagrams, two problems arise. Firstly, the pieces one sees combinatorially by shrinking edges on the graph have Hodge structures which are themselves mixed rather than pure. And secondly, it is not possible in general to make the intersection between the polar locus $X$ and the simplex at infinity transverse by simply blowing up faces of the simplex. Presumably, therefore, the analog of the duality used above

$$
H^{2}\left(P-E, \mathfrak{h}^{0}\right)^{\vee} \cong H^{2}\left(\mathbb{G}_{m}^{2}, E^{0}\right)(2)
$$

is not valid in general, which means that the link between the Feynman integral and polylogarithms is more tenuous. 
Remark 4.4. - In section 7, formula (4.27) is rederived in terms of regulator currents, as a byproduct of the $K$-theoretic approach to the inhomogeneous Picard-Fuchs equation. The relationship between these currents and $C$ (in (4.25)) is explained in [KLM].

\section{PART III \\ THE LOCAL MIRROR SYMMETRY}

In this part we revisit the approach of [CKYZ] to local mirror symmetry, by semistably degenerating a family of elliptically-fibered Calabi-Yau 3-folds $\mathrm{X}_{z_{0}, \underline{z}}$ (defined by (5.1)) to a singular compactification of the local Hori-Vafa 3-fold

$$
Y_{\underline{z}}:=\left\{1-s\left(\xi_{1}^{2} x+\xi_{2}^{2} y+\xi_{3}^{2}\right)\left(1+x^{-1}+y^{-1}\right)+u v=0\right\} \subset\left(\mathbb{C}^{*}\right)^{2} \times \mathbb{C}^{2}
$$

\section{B-model}

In this section we describe the degeneration from a compact Calabi-Yau 3-fold X to the local Hori-Vafa model Y (which is a noncompact Calabi-Yau 3-fold) [HV]. The main point is that the third homology of $\mathrm{Y}$ matches the invariant part of the limiting mixed Hodge structure of $H^{3}(\mathrm{X})$ (Theorem 5.3). Comparing with the limiting mixed Hodge structure of the A-model in the next section will allow us to deduce a strong form of local mirror symmetry - equality of variations of $\mathbb{Q}$-mixed Hodge structure — which implies the conjecture 5.1 ${ }^{(6)}$ from [DK], see Theorem 6.1.

5.1. Laurent polynomial. - Choose a reflexive polytope $\Delta \subset \mathbb{R}^{2}$, with polar polytope $\Delta^{\circ}$, and write $r=\left|\partial \Delta \cap \mathbb{Z}^{2}\right|, r^{\circ}=\left|\partial \Delta^{\circ} \cap \mathbb{Z}^{2}\right|$, and $\nu\left(\leq r, r^{\circ}\right)$ for the common number of edges and vertices of both $\Delta$ and $\Delta^{\circ}$. The toric surface associated to $\Delta$ is constructed from the fan on (the vertices of) $\Delta^{\circ}$, and has canonical desingularization $\mathbb{P}_{\Delta} \rightarrow \check{\mathbb{P}}_{\Delta}$ arising from the fan on all integer points of $\partial \Delta^{\circ}$. Writing $\partial \Delta \cap \mathbb{Z}^{2}=$ $\left\{\underline{m}^{(j)}\right\}_{j=1}^{r}$, the general Laurent polynomial with Newton polytope $\Delta$ is

$$
f_{\underline{a}}(x, y):=a_{0}+\sum_{j=1}^{r} a_{j} x^{m_{1}^{(j)}} y^{m_{2}^{(j)}}
$$

(with $a_{j} \in \mathbb{C}^{*}$ ). The compactification of $\left\{f_{\underline{a}}(x, y)=0\right\} \subset \mathbb{G}_{m}^{2}$ in $\mathbb{P}_{\Delta}$ yields (for general $\left.\left\{a_{j}\right\}\right)$ a smooth elliptic curve $E_{\underline{a}}$.

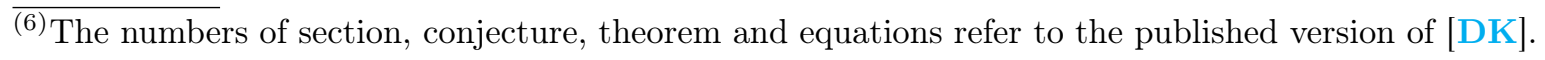


Jumping up two dimensions, in coordinates $\left(x_{1}, x_{2}, x_{3}, x_{4}\right)=(x, y, u, v)$ on $\mathbb{G}_{m}^{4}$, we set

$$
F:=\mathrm{a}+\mathrm{b} u^{2} v^{-1}+\mathrm{c} u^{-1} v+u^{-1} v^{-1} f_{\underline{a}}(x, y)
$$

(with $\mathrm{a}, \mathrm{b}, \mathrm{c} \in \mathbb{C}^{*}$ ). Its Newton polytope

$$
\hat{\Delta}:=\Delta(F)=\text { hull }\{(0,0,2,-1),(0,0,-1,1), \Delta \times(-1,-1)\}
$$

is reflexive since its polar

$$
\hat{\Delta}^{\circ}=\operatorname{hull}\left\{(0,0,1,0),(0,0,0,1), 6 \Delta^{\circ} \times(-2,-3)\right\}
$$

is integral. Let $\check{\mathbb{P}}_{\hat{\Delta}}$ be the toric 4-fold associated to $\hat{\Delta}$ (i.e. to the fan on $\hat{\Delta}^{\circ}$ ), and $\mathbb{P}_{\hat{\Delta}} \rightarrow \check{\mathbb{P}}_{\hat{\Delta}}$ a maximal projective crepant partial (MPCP) desingularization (arising from the fan on a maximal triangulation $\left.\operatorname{tr}\left(\partial \hat{\Delta}^{\circ}\right)\right)$. For general a, b, c, $\underline{a}$, the compactification

$$
\mathrm{X}_{F}:=\overline{\{F=0\}} \subset \mathbb{P}_{\hat{\Delta}}
$$

is a smooth Calabi-Yau 3-fold.

To describe the coordinates about the large complex structure limit in the simplified polynomial moduli space, consider the cone $\mathcal{L}$ of $\mathbb{Z}_{\geq 0}$-relations on the $\left\{\underline{m}^{(j)}\right\}$. If it is simplicial and smooth with basis $\left\{\underline{\ell}^{(i)}\right\}_{i=1}^{r-2}$, the coordinates are

$$
z_{0}:=\frac{a_{0} \mathrm{~b}^{2} \mathrm{c}^{3}}{\mathrm{a}^{6}} \text { and } z_{i}:=\frac{\prod_{j=1}^{r} a_{j}^{\ell_{j}^{(i)}}}{a_{0}^{\sum_{j} \ell_{j}^{(i)}}}(i=1, \ldots, r-2) .
$$

Otherwise, there are more $z_{i}$ 's (with relations), though $z_{0}$ remains the same; we will explain how to deal with this complication for the sunset case at the end of the section.

What follows is a study of the degeneration of $\mathrm{X}_{F}$ as $z_{0} \rightarrow 0$.

5.2. Maximal projective crepant partial desingularization. $-\mathbb{P}_{\hat{\Delta}}$ is not unique, and for an arbitrary choice of triangulation $\operatorname{tr}\left(\partial \hat{\Delta}^{\circ}\right)$ may have isolated terminal singularities. We shall now describe (and fix) a triangulation which results in a smooth $\mathbb{P}_{\hat{\Delta}}$.

The integral points on $\hat{\Delta}^{\circ}$ are

$$
\begin{gathered}
(0,0,1,0),(0,0,0,1),\left(6 \Delta^{\circ}\right)_{\mathbb{Z}} \times(-2,-3),\left(4 \Delta^{\circ}\right)_{\mathbb{Z}} \times(-1,-2), \\
\left(3 \Delta^{\circ}\right)_{\mathbb{Z}} \times(-1,-1),\left(2 \Delta^{\circ}\right)_{\mathbb{Z}} \times(0,-1), \Delta_{\mathbb{Z}}^{\circ} \times(0,0) .
\end{gathered}
$$

It has $\nu$ facets of the form

$$
\operatorname{hull}\left\{6 e_{i}^{\circ} \times(-2,-3),(0,0,1,0),(0,0,0,1)\right\}=: \mathfrak{f}_{\mathrm{a}, i}^{\circ}
$$

where $\left\{e_{i}^{\circ}\right\}_{i=1}^{\nu}$ are edges of $\Delta^{\circ}$, and two of the form

$$
\begin{aligned}
& \operatorname{hull}\left\{6 \Delta^{\circ} \times(-2,-3),(0,0,1,0)\right\}=: \mathfrak{f}_{\mathrm{b}}^{\circ} \\
& \operatorname{hull}\left\{6 \Delta^{\circ} \times(-2,-3),(0,0,0,1)\right\}=: \mathfrak{f}_{\mathrm{c}}^{\circ} .
\end{aligned}
$$

The decomposition of these facets into elementary tetrahedra proceeds in four steps: 
Step 1:- For each $\mathbb{Z}$-point $\underline{w} \in\left(\partial \Delta^{\circ}\right)_{\mathbb{Z}}$, draw the half-space

$$
\mathbb{H}_{\underline{w}}:=\overrightarrow{0 . \underline{w}} \times \mathbb{C}_{u, v}^{2}
$$

through it. This subdivides the facets.

Step 2:- Up to unimodular transformation, the resulting "slices" of $\mathfrak{f}_{\mathrm{b}}^{\circ}$ resp. $\mathfrak{f}_{\mathrm{c}}^{\circ}$ are

$$
\operatorname{hull}\left\{(0,0,-2,-3),(6,0,-2,-3),(0,6,-2,-3), \quad \begin{array}{c}
(0,0,1,0) \\
\text { resp. }(0,0,0,1)
\end{array}\right\} \text {. }
$$

To triangulate the first one (second is similar): first decompose it into

$$
\mathfrak{f}_{\mathfrak{A}}=\operatorname{hull}\{\underbrace{(3,0,-1,-1),(0,3,-1,-1),(0,0,-1,-1)}_{\text {hull }=: \mathfrak{p}_{\mathfrak{A}}},(0,0,0,1)\}
$$

and

$$
\mathfrak{f}_{\mathfrak{B}}=\operatorname{hull}\{\mathfrak{p}_{\mathfrak{A}}, \underbrace{(6,0,-2,-3),(0,6,-2,-3),(0,0,-2,-3)}_{\text {hull }=: \mathfrak{p}_{\mathfrak{B}}}\} ;
$$

on $\mathfrak{p}_{\mathfrak{A}}$ and $\mathfrak{p}_{\mathfrak{B}}$, draw all the integral horizontal, vertical, and anti-diagonal $(x+y=$ $k$ ) lines; then for $\mathfrak{f}_{\mathfrak{A}}$, complete the resulting triangles to tetrahedra with vertex at $(0,0,0,1)$; for $\mathfrak{f}_{\mathfrak{B}}$, further subdivide into the 4 tetrahedra

$$
\begin{aligned}
& \operatorname{hull}\{(0,0,-1,-1),(0,0,-2,-3),(3,-,-2,-3),(0,3,-2,-3)\} \\
& \operatorname{hull}\{(0,3,-1,-1),(0,3,-2,-3),(3,3,-2,-3),(0,6,-2,-3)\} \\
& \operatorname{hull}\{(3,0,-1,-1),(3,0,-2,-3),(6,0,-2,-3),(3,3,-2,-3)\} \\
& \operatorname{hull}\{(0,0,-1,-1),(3,0,-1,-1),(0,3,-1,-1),(3,3,-2,-3)\}
\end{aligned}
$$

(treating these as with $\mathfrak{f}_{\mathfrak{A}}$ ) and the 2 "skew" tetrahedra

$$
\begin{aligned}
& \operatorname{hull}\{(0,0,-1,-1),(0,3,-1,-1),(0,3,-2,-3),(3,3,-2,-3)\} \\
& \operatorname{hull}\{(0,0,-1,-1),(3,0,-1,-1),(3,0,-2,-3),(3,3,-2,-3)\}
\end{aligned}
$$

(which get subdivided into elementary tetrahedra of the form

$$
\operatorname{hull}\{(a, 0,-1,-1),(a+1,0,-1,-1),(3, b,-2,-3),(3, b+1,-2,-3)\}) .
$$

Step 3:- For the $\mathfrak{f}_{\mathrm{a}, i}^{\circ}$, it will not matter which triangulation we choose. Two of the 2 -faces of $\mathfrak{f}_{\mathrm{a}, i}^{\circ}$ already receive a triangulation from Step 2. The other 2 may be startriangulated with centers of the form $\underline{v} \times(0,0), \underline{v} \in \Delta_{\mathbb{Z}}^{\circ}$. Any 3-triangulation completing this will do.

Step 4:- One checks that all of the tetrahedra in this triangulation are regular, i.e. the determinants of their vertices are \pm 1 . This is not always possible for a general 4-dimensional reflexive polytope, and shows that $\mathbb{P}_{\hat{\Delta}}$ is smooth. 
5.3. Elliptic fibration. - Write $\Sigma_{\Delta}\left(\operatorname{resp} . \Sigma_{\hat{\Delta}}\right)$ for the fan on $\partial \Delta_{\mathbb{Z}}^{\circ}$ (resp. on the triangulation $\left.\operatorname{tr}\left(\partial \hat{\Delta}^{\circ}\right)\right)$. By Step 1 in $\S 5.2$, we have a map of fans $\Sigma_{\hat{\Delta}} \rightarrow \Sigma_{\Delta}$ hence a diagram

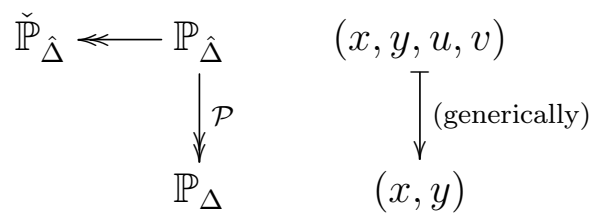

with $\mathbb{P}$ a morphism. The components of $\mathbb{D}_{\hat{\Delta}}:=\mathbb{P}_{\hat{\Delta}} \backslash \mathbb{G}_{m}^{4}$ not lying over a component of $\mathbb{D}_{\Delta}=\mathbb{P}_{\Delta} \backslash \mathbb{G}_{m}^{2}$, are the ones dual to $\mathbb{Z}$-points of $\hat{\Delta}^{\circ}$ with first two coordinates 0 (except the origin). These are $(0,0) \times$

$$
(1,0),(0,1),(-2,-3),(-1,-2),(-1,-1),(0,-1),
$$

the fan on which produces the toric surface $\mathbb{P}_{W}$, which is a desingularization of $\mathbb{W} \mathbb{P}(1,2,3)$. So the "generic" fiber of $\mathcal{P}$ is $\mathbb{P}_{W}$, the "correct" toric surface in which to compactify the (generalized) Weierstrass elliptic curve $F\left(x_{0}, y_{0}, u, v\right)=0$ (where $x_{0}, y_{0} \in \mathbb{C}^{*}$ ).

We now describe the induced elliptic fibration

$$
\mathrm{X}_{F} \stackrel{\rho}{\rightarrow} \mathbb{P}_{\Delta} .
$$

Write

with compactification $E_{\underline{a}} \subset \mathbb{P}_{\Delta}$; and

$$
E_{\underline{a}}^{*}=\left\{f_{\underline{a}}(x, y)=0\right\} \cap \mathbb{G}_{m}^{2},
$$

$$
D_{\underline{a}, z_{0}}^{*}=\left\{f_{\underline{a}}(x, y)=\frac{a_{0}}{2 \cdot 6^{3} z_{0}}\right\} \cap \mathbb{G}_{m}^{2},
$$

with compactification $D_{a, z_{0}} \subset \mathbb{P}_{\Delta}$. We have $E_{a} \cap D_{a, z_{0}}=E_{a} \cap \mathbb{D}_{\Delta}=D_{a, z_{0}} \cap \mathbb{D}_{\Delta}=: \mathbb{B}_{\Delta, a}$ (which consists of $r$ points) for the base locus of the pencil $f_{a}(x, y)=\lambda, \lambda \in \mathbb{P}^{1}(\mathbb{C})$. The 1-dimensional fibers of $\rho$ are:

- over $\mathbb{G}_{m}^{2} \backslash\left\{D_{a, z_{0}}^{*} \cup E_{a}^{*}\right\}$, a smooth elliptic curve " $\mathcal{E}$ ";

- over $E_{\underline{a}}^{*}$, type $I_{1}$ (nodal rational curve) with node at $(u, v)=(0,0)$;

- over $D_{\underline{a}, z_{0}}^{*}$, type $I_{1}$ with node at $(u, v)=\left(\frac{-\mathrm{a}^{3}}{12 \mathrm{bc}^{2}}, \frac{\mathrm{a}^{2}}{6 \mathrm{bc}}\right)$; and

- over $\mathbb{D}_{\Delta} \backslash\left\{\operatorname{sing}\left(\mathbb{D}_{\Delta}\right) \cup \mathbb{B}_{\Delta}\right\}$, type $I I^{*}\left(E_{8}\right.$ configuration $)$.

Indeed, the local system on $\mathbb{G}_{m}^{2} \backslash\left\{E_{\underline{a}}^{*} \cup D_{\underline{a}, z_{0}}^{*}\right\}$ is the pullback (by $\lambda=f_{\underline{a}}(x, y) \cdot 2$. $\left.6^{3} \mathrm{~b}^{2} \mathrm{c}^{3} / \mathrm{a}^{6}\right)$ of the fibered wise first homology group $H_{1}$ of the family

$$
\frac{\lambda \mathrm{a}^{6}}{2 \cdot 6^{3} \mathrm{~b}^{2} \mathrm{c}^{3}}+\mathrm{a} u v+\mathrm{b} u^{3}+\mathrm{c} v^{2}=0
$$

in $\mathbb{P}_{W}$, with singular fibers at 0 of type $I_{1}, 1$ of type $I_{1}, \infty$ of type $I I^{*}$. On $\mathbb{C} \backslash\{(-\infty, 0] \cup$ $[1, \infty)\}$, we have a basis $\{\alpha, \beta\}$ of 1 -cycles (for the local system) with monodromies $\left(\begin{array}{ll}1 & 1 \\ 0 & 1\end{array}\right)\left[\operatorname{resp} .\left(\begin{array}{cc}1 & 0 \\ -1 & 1\end{array}\right),\left(\begin{array}{cc}0 & -1 \\ 1 & 1\end{array}\right)\right]$ about 0 [resp. 1, $\left.\infty\right]$; accordingly, we shall write $\alpha$ resp. $\beta$ for the vanishing cycles of the pullback local system at $E_{\underline{a}}^{*}$ resp. $D_{\underline{a}, z_{0}}^{*}$. 
Over $\mathbb{B}_{\Delta, \underline{a}}$ and $\mathbb{S}_{\Delta}:=\operatorname{sing}\left(\mathbb{D}_{\Delta}\right)$, the fibers of $\rho$ have dimension 2 . The components are obtained by taking preimages of solutions to edge- and 2-face-polynomials of $F$ under the blowups used to produce $\mathbb{P}_{\hat{\Delta}}$ from $\check{\mathbb{P}}_{\hat{\Delta}}$. These preimages are hypersurfaces in components of $\mathbb{D}_{\hat{\Delta}}$ corresponding to integer points of $\hat{\Delta}^{\circ}$ (not in its interior or that of its facets) lying in one of the open half-spaces $\mathbb{H}_{\underline{w}}$ (for $\mathbb{B}_{\Delta, \underline{a}}$ ) or in between two of them (for $\mathbb{S}_{\Delta}$ ).

For $\mathbb{B}_{\Delta, \underline{a}}$, the sole contribution comes from the points of the form $\underline{v} \times(0,0)$ where $\underline{v}$ is a vertex of $\Delta^{\circ}$ (dual to an edge $e_{\underline{v}}$ of $\left.\Delta\right)$. These belong to the interior of a 2 -face hull $\{6 \underline{v} \times(-2,-3),(0,0,1,0),(0,0,0,1)\}$ which is dual to the edge $e_{\underline{v}} \times(-1,-1)$ of $\hat{\Delta}$. When the corresponding 1-dimensional subspace of $\check{\mathbb{P}}_{\hat{\Delta}}$ is blown up to a 3-dimensional one in $\mathbb{P}_{\hat{\Delta}}$, the equation is inherited from the edge polynomial of $F$, which cuts out a point (or points) of $\mathbb{B}_{\Delta, a}$. Since this blowup arises from the star subdivision of Step 3 from $\S 5.2$, we conclude that the fiber over said point is a copy of $\mathbb{P}_{W}$.

For $\mathbb{S}_{\Delta}$, there are contributions from all the points of the form

$$
\begin{array}{cc}
\left(2 \partial \Delta^{\circ}\right)_{\mathbb{Z}} \backslash 2 \partial \Delta_{\mathbb{Z}}^{\circ} \times(0,-1), & \left(2 \partial \Delta^{\circ}\right)_{\mathbb{Z}} \backslash 3 \partial \Delta_{\mathbb{Z}}^{\circ} \times(-1,-1), \\
\left(2 \partial \Delta^{\circ}\right)_{\mathbb{Z}} \backslash 4 \partial \Delta_{\mathbb{Z}}^{\circ} \times(-1,-2), & \left(6 \Delta^{\circ}\right)_{\mathbb{Z}} \backslash 6 \Delta_{\mathbb{Z}}^{\circ} \times(-2,-3) .
\end{array}
$$

Unimodular transformation maps any edge of $\operatorname{tr}\left(\partial \Delta^{\circ}\right)$ to $[(0,1),(1,0)]$, hence for a given point $p \in \mathbb{S}_{\Delta}$ (dual to that edge), one easily sees that $\rho^{-1}(p)$ consists of 21 rational surfaces.

Remark 5.1. - The fibration $\rho$ has an obvious section, given by the intersection of $\mathrm{X}_{F}$ with the component of $\mathbb{D}_{\hat{\Delta}}$ indexed by the point $(0,0,-2,-3) \in \hat{\Delta}_{\mathbb{Z}}^{\circ}$. In a generic fiber this is the usual "point at $\infty$ " in the Weierstrass elliptic curve.

5.4. Middle homology of $X_{F}$. - We shall work henceforth under the assumption that $a_{i>0}$ and $z_{0}$ are sufficiently small. Write $\ell(\theta)$ resp. $\ell^{*}(\theta)$ for the number of integral points in a polytope $\theta$ resp. its interior. We have

$$
h^{3,0}\left(\mathrm{X}_{F}\right)=\ell^{*}(\hat{\Delta})=1
$$

since $\hat{\Delta}$ is reflexive, with the (unique up to scale) holomorphic 3 -form given by

$$
\Omega_{F}=\frac{1}{(2 \pi \mathbf{i})^{3}} \operatorname{Res}_{\mathrm{X}_{F}}\left(\frac{d x / x \wedge d y / y \wedge d u / u \wedge d v / v}{F}\right) \in \Omega^{3}\left(\mathrm{X}_{F}\right)
$$


We have also the Batyrev formula [Bat]

$$
\begin{aligned}
h^{2,1}\left(\mathrm{X}_{F}\right) & =\ell(\hat{\Delta})-\sum_{\substack{\sigma \text { facet } \\
\text { of } \hat{\Delta}}} \ell^{*}(\sigma)+\sum_{\begin{array}{c}
\theta \text { 2-face } \\
\text { of } \hat{\Delta}
\end{array}} \ell^{*}(\theta) \ell^{*}\left(\theta^{\circ}\right)-5 \\
& =\{\ell(\Delta)+6\}-1+2+0-5 \\
& =\ell(\Delta)-2 \\
& =r-1 .
\end{aligned}
$$

Now we shall use the structure of the elliptic fibration to exhibit a basis of $H_{3}\left(\mathrm{X}_{F}, \mathbb{Q}\right)$. (In what follows we often drop subscripts $\underline{a}, z_{0}, F$, etc.; moreover, some steps are only sketched).

The basic observation is that

$$
\mathcal{K}_{E}:=\operatorname{ker}\left\{H_{1}\left(E^{*}\right) \rightarrow H_{1}\left(\mathbb{G}_{m}^{2}\right)\right\}=\operatorname{ker}\left\{H_{1}\left(E^{*}\right) \rightarrow H_{1}\left(\mathbb{G}_{m}^{2} \backslash D^{*}\right)\right\}
$$

and

$$
\mathcal{K}_{D}:=\operatorname{ker}\left\{H_{1}\left(D^{*}\right) \rightarrow H_{1}\left(\mathbb{G}_{m}^{2}\right)\right\}=\operatorname{ker}\left\{H_{1}\left(D^{*}\right) \rightarrow H_{1}\left(\mathbb{G}_{m}^{2} \backslash E^{*}\right)\right\}
$$

are $(r-1)$-dimensional spaces. (For instance, to see the second equality for $\mathcal{K}_{E}$, take $z_{0}$ small enough that $D$ lies inside an $\epsilon$-neighborhood $U$ of $\mathbb{D}_{\Delta}$, and replace $E^{*}$ by $E \backslash\{E \cap U\}$.) Moreover, we have two obvious 3-cycles $\mathcal{T}_{\alpha}$ and $\mathcal{T}_{\beta}$ consisting of parallel translates of $\alpha$ resp. $\beta$ over $\mathbb{T}:=\{|x|=|y|=1\}$. We will show that, together with these, certain cycles built from $\mathcal{K}_{E}$ and $\mathcal{K}_{D}$ yield $2 r$ independent 3 -cycles on $\mathrm{X}_{F}$.

To construct these cycles, let $\{\varphi\}=\left\{\left\{\varphi_{0}^{(i)}\right\}_{i=1}^{r-2}, \varphi_{1}\right\} \subset \mathcal{K}_{E}$ be a basis (with $\left\{\varphi_{0}^{(i)}\right\}$ all being homologous to one $\varphi_{0}$ on $E$ ). Choose for each $\varphi$ a 2-chain $\Gamma_{\varphi} \subset \mathbb{G}_{m}^{2} \backslash D^{*}$ with $\partial \Gamma_{\varphi}=\varphi$; and let $\mathcal{M}_{\alpha}(\varphi)$ be a continuous family of 1-cycles of class $[\alpha]$ over $\Gamma_{\varphi}$ collapsing to a point over $p$. We take $\Phi_{E} \subset H_{3}\left(\pi^{-1}\left(\mathbb{G}_{m}^{2} \backslash D^{*}\right)\right)$ to be the span of the $\left\{\left[\mathcal{M}_{\alpha}(\varphi)\right]\right\}$, and similarly $\Phi_{D}=\operatorname{span}\left\{\left[\mathcal{M}_{\beta}(\varphi)\right]\right\}_{\varphi \in \mathcal{K}_{D}} \subset H_{3}\left(\rho^{-1}\left(\mathbb{G}_{m}^{2} \backslash E^{*}\right)\right)$.

We may compute $H_{3}^{D}:=H_{3}\left(\rho^{-1}\left(\mathbb{G}_{m}^{2} \backslash D^{*}\right)\right)$ via the relative homology sequence

$$
\begin{aligned}
\cdots \rightarrow H_{3}\left(\rho^{-1} E^{*}\right) \stackrel{\psi_{E}}{\rightarrow} H_{3}^{D} & \rightarrow H_{3}\left(\rho^{-1}\left(\mathbb{G}_{m}^{2} \backslash D^{*}\right), \pi^{-1} E^{*}\right) \\
& \stackrel{\theta_{E}}{\rightarrow} H_{2}\left(\rho^{-1} E^{*}\right) \rightarrow \cdots,
\end{aligned}
$$

in which

$$
\operatorname{im}\left(\psi_{E}\right) \cong \operatorname{im}\left\{H_{1}\left(E^{*}\right) \rightarrow H_{1}\left(\mathbb{G}_{m}^{2} \backslash D^{*}\right)\right\} \otimes[\mathcal{E}] \underset{n . c .}{\cong} H_{1}\left(\mathbb{G}_{m}^{2}\right) .
$$

The second isomorphism is not canonical. Writing $\mathcal{H}_{1}$ for $\left(R^{1} \rho_{*} \mathbb{Q}\right)^{\vee}$, the Leray spectral sequence yields

$$
\begin{aligned}
0 & \rightarrow H_{1}\left(\mathbb{G}_{m}^{2} \backslash D^{*}, E^{*}\right) \otimes[\mathcal{E}] \\
& \rightarrow \operatorname{ker}\left(\theta_{E}\right) \rightarrow H_{2}\left(\mathbb{G}_{m}^{2} \backslash D^{*}, E^{*} ; \mathcal{H}_{1}\right) \stackrel{\theta_{E}^{\prime}}{\rightarrow} H_{1}\left(E^{*}, \mathcal{H}_{1} /\langle\alpha\rangle\right)
\end{aligned}
$$


so that (5.5) becomes

$$
0 \rightarrow H_{1}\left(\mathbb{G}_{m}^{2} \backslash D^{*}\right) \otimes[\mathcal{E}] \rightarrow H_{3}^{D} \rightarrow \operatorname{ker}\left(\theta_{E}^{\prime}\right) \rightarrow 0 .
$$

Using the exact sequences

$$
\begin{gathered}
0 \rightarrow H_{2}\left(\mathbb{G}_{m} \backslash D^{*}\right) \rightarrow H_{2}\left(\mathbb{G}_{m}^{2} \backslash D^{*}, E^{*}\right) \rightarrow \mathcal{K}_{E} \rightarrow 0 \\
0 \rightarrow H_{1}\left(D^{*}\right) \stackrel{\text { Tube }}{\rightarrow} H_{2}\left(\mathbb{G}_{m}^{2} \backslash D^{*}\right) \rightarrow H_{2}\left(\mathbb{G}_{m}^{2}\right) \rightarrow 0,
\end{gathered}
$$

and writing $\mathrm{T}:=\mathbb{Q}\left\langle\left[\mathcal{T}_{\alpha}\right],\left[\mathcal{T}_{\beta}\right]\right\rangle \subset H_{3}^{D}$ and $\Psi_{D}:=\operatorname{Tube}\left(H_{1}\left(D^{*}\right)\right) \otimes[\beta]$, one can then show (with some work) that $T \oplus \Psi_{D} \oplus \Phi_{E}$ maps isomorphically to $\operatorname{ker}\left(\theta_{E}^{\prime}\right)$. Repeat this whole argument with $D$ and $E$ (and $\alpha$ and $\beta$ ) swapped to compute $H_{3}^{E}$.

Next, one explicitly checks that $H_{2}\left(\rho^{-1}\left(\mathbb{G}_{m}^{2} \backslash\left\{D^{*} \cup E^{*}\right\}\right)\right)=: H_{2}^{D E}$ injects into $H_{2}^{D} \oplus$ $H_{2}^{E}$, so that

$$
H_{3}\left(\rho^{-1} \mathbb{G}_{m}^{2}\right) \cong \frac{H_{3}^{D} \oplus H_{3}^{E}}{\operatorname{im}\left\{H_{3}^{D E}\right\}} .
$$

The Leray spectral sequence for $\rho$ yields

$$
\begin{aligned}
0 & \rightarrow H_{1}\left(\mathbb{G}_{m}^{2} \backslash\left\{D^{*} \cup E^{*}\right\}\right) \otimes[\mathcal{E}] \\
& \rightarrow H_{3}^{D E} \rightarrow H_{2}\left(\mathbb{G}_{m}^{2} \backslash\left\{D^{*} \cup E^{*}\right\} ; \mathcal{H}_{1}\right) \rightarrow 0,
\end{aligned}
$$

which (comparing with (5.7)) breaks the computation of the quotient (5.8) into two pieces: for the "left-hand" piece, we have

$$
\frac{H_{1}\left(\mathbb{G}_{m}^{2} \backslash D^{*}\right) \oplus H_{1}\left(\mathbb{G}_{m}^{2} \backslash E^{*}\right)}{\operatorname{im}\left\{H_{1}\left(\mathbb{G}_{m}^{2} \backslash\left\{D^{*} \cup E^{*}\right\}\right)\right\}} \otimes[\mathcal{E}] \cong H_{1}\left(\mathbb{G}_{m}^{2}\right) \otimes[\mathcal{E}]
$$

For the "right-hand" piece, the quotient of $\operatorname{ker}\left(\theta_{E}^{\prime}\right)$ by the right-hand term of (5.9), which is an extension

$$
0 \rightarrow \Psi_{D} \oplus \Psi_{E} \rightarrow H_{2}\left(\mathbb{G}_{m}^{2} \backslash\left\{D^{*} \cup E^{*}\right\} ; \mathcal{H}_{1}\right) \rightarrow \mathrm{T} \rightarrow 0,
$$

is evidently isomorphic to $\Phi_{E} \oplus \Phi_{D} \oplus \mathrm{T}$.

Finally, we consider the cohomology of the normal crossing divisor $\rho^{-1} \mathbb{D}_{\Delta}=\cup \mathcal{R}_{i}$; here the $\mathcal{R}_{i}$ are rational surfaces (meeting along $\mathbb{P}^{1}$ 's) indexed by $\mathbb{Z}$-points of $\hat{\Delta}^{\circ}$ with $(x, y) \neq(0,0)$ and not in the interior of a facet. By studying the part of the 2-skeleton of $\operatorname{tr}\left(\partial \hat{\Delta}^{\circ}\right)$ meeting these points, we compute the spectral sequence converging to $H^{*}\left(\rho^{-1} \mathbb{D}_{\Delta}\right)$, with $E_{1}$ page

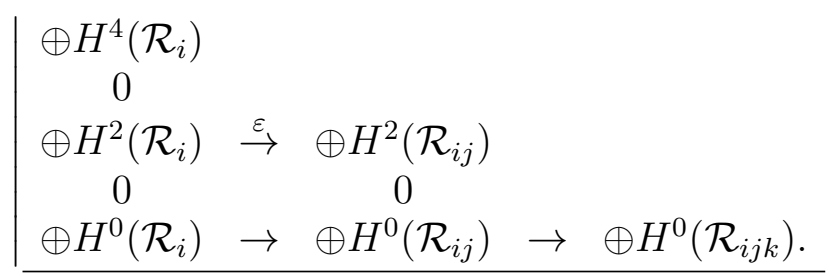

The cohomology ranks of the bottom row are just the Betti numbers 1, 1,0 of the 2-skeleton, so that $H^{1}(\mathbb{D}) \rightarrow H^{1}\left(\rho^{-1} \mathbb{D}\right) \stackrel{\text { Tube }}{\rightarrow} H_{4}\left(\mathrm{X} \backslash \rho^{-1} \mathbb{D}_{\Delta}\right)$ are isomorphisms (all of 
rank 1, with Tube hitting $\mathbb{T} \otimes[\mathcal{E}])$. One also deduces from this that $\operatorname{ker}\left\{\oplus H_{2}\left(\mathcal{R}_{i j}\right) \rightarrow\right.$ $\left.\oplus H_{4}\left(\mathcal{R}_{i}\right)\right\}$, hence coker $(\varepsilon)$ and $H^{3}\left(\rho^{-1} \mathbb{D}_{\Delta}\right)$, have rank 1 ; it follows that $H^{3}\left(\rho^{-1} \mathbb{D}_{\Delta}\right) \stackrel{\text { Tube }}{\rightarrow}$ $H_{2}\left(\mathrm{X} \backslash \rho^{-1} \mathbb{D}_{\Delta}\right)$ is injective, with Tube hitting $\mathbb{T} \otimes[\mathrm{pt}$.$] . Moreover, we have$

$$
\left\{\begin{array}{l}
\operatorname{rank}\left(\oplus H^{4}\left(\mathcal{R}_{i}\right)\right)=30 r^{\circ}+\nu \\
\operatorname{rank}\left(\oplus H^{2}\left(\mathcal{R}_{i}\right)\right)=120 r^{\circ}+5 \nu \\
\operatorname{rank}\left(\oplus H^{2}\left(\mathcal{R}_{i j}\right)\right)=90 r^{\circ}+4 \nu
\end{array}\right.
$$

and so conclude that $h^{2}\left(\pi^{-1} \mathbb{D}_{\Delta}\right)=120 r^{\circ}+5 \nu-\left(90 r^{\circ}+4 \nu\right)+1=30 r^{\circ}+\nu+1$. Now by Batyrev [Bat]

$$
\begin{aligned}
h^{4}(\mathrm{X}) & =h^{2}(\mathrm{X})=\ell\left(\hat{\Delta}^{\circ}\right)-\sum_{\substack{\sigma^{\circ} \text { facet } \\
\text { of } \hat{\Delta}^{\circ}}} \ell^{*}\left(\sigma^{\circ}\right)+\sum_{\substack{\theta^{\circ} 2 \text {-face } \\
\text { of } \hat{\Delta}^{\circ}}} \ell^{*}\left(\theta^{\circ}\right) \ell^{*}(\theta)-5 \\
& =\left(31 r^{\circ}+7\right)-\left(r^{\circ}-\nu+3\right)-5 \\
& =30 r^{\circ}+\nu-1 .
\end{aligned}
$$

By the exact sequence

$$
0 \rightarrow H_{4}(\mathrm{X}) \rightarrow H^{2}\left(\rho^{-1} \mathbb{D}_{\Delta}\right) \stackrel{\text { Tube }}{\rightarrow} H_{3}\left(X \backslash \rho^{-1} \mathbb{D}_{\Delta}\right) \rightarrow H_{3}(\mathrm{X}) \rightarrow 0
$$

we now have $\operatorname{rank}($ Tube $)=h^{2}\left(\rho^{-1} \mathbb{D}_{\Delta}\right)-h^{4}(\mathrm{X})=2$. Since $H_{1}\left(\mathbb{G}_{m}^{2}\right) \otimes[\mathcal{E}]$ is evidently in the image of Tube, this is im(Tube) and thus

$$
H_{3}(\mathrm{X})=\Phi_{D} \oplus \Phi_{E} \oplus \mathrm{T} \text {. }
$$

5.5. Degeneration as $z_{0} \rightarrow 0$. - We shall need to replace $z_{0}$ by $t$, which amounts to pullback by $t \mapsto t^{5}$. Set $\mathrm{b}=\mathrm{c}=t, \mathrm{a}=1$ in (5.1). Write $\mathrm{X}_{\underline{a}, t}$ (or just $\mathrm{X}_{t}$ ) for the corresponding Calabi-Yau 3-fold; fix an $\underline{a}$ and disk $\Delta$ about 0, such that $\mathcal{X}_{\underline{a}} \rightarrow \Delta$ (with fibers $\left.\mathrm{X}_{\underline{a}, t}\right)$ is smooth away from $\{0\}$. For the singular fibers write

$$
\mathrm{X}_{\underline{a}, 0}=\cup_{i \geq 0} W_{i}
$$

where

$$
W_{0}=\bar{Y}:=\overline{\left\{f_{\underline{a}}(x, y)+u v=0\right\}} \subset \mathbb{P}_{\hat{\Delta}}
$$

and $W_{i>0}$ are the components of $\mathbb{D}_{\hat{\Delta}}$ corresponding to integer points of $\hat{\Delta}^{\circ}$ contained in the interiors of the 2 -face $6 \Delta^{\circ} \times(-2,-3)$ and of the facets $\mathfrak{f}_{\mathrm{b}}^{\circ}$ and $\mathfrak{f}_{\mathrm{c}}^{\circ}$. Write $I_{\Delta^{\circ}}$ for the index (sub)set corresponding just to the interior points of $6 \Delta^{\circ} \times(-2,-3)$.

The singular locus of the total space is contained in $\mathrm{X}_{\underline{a}, 0}$; more precisely, it consists of the intersections $W_{i} \cap W_{j} \cap \mathrm{X}_{\underline{a}, t \neq 0} \cong \mathbb{P}^{1}$ with $i, j \in I_{\Delta^{\circ}}$. Let $\mathbb{P}_{\hat{\Delta}}^{\prime} \stackrel{B}{\rightarrow} \mathbb{P}_{\hat{\Delta}}$ denote the blow-up along the smooth rational surfaces $\left\{W_{i} \cap \mathrm{X}_{\underline{a}, t \neq 0}\right\}_{i \in I_{\Delta^{\circ}}}$, in any order, and $\mathcal{X}^{\prime} \rightarrow \mathcal{X}$ the proper transform under $B \times \mathrm{id}_{\Delta}$. Note that $\overline{\mathcal{X}}^{\prime}$ is smooth, with fibers over $\Delta^{*}$ unchanged, and $\mathrm{X}_{\underline{a}, 0}^{\prime}=\cup W_{i}^{\prime}$ having no additional irreducible components. Indeed, 
the only change is that some irreducible components of $\mathrm{X}_{0}$ have been blown up along some $\mathbb{P}^{1}$ 's. Write $\mathbb{D}_{\hat{\Delta}}^{\prime}, \bar{Y}^{\prime}$, etc. for proper transforms.

Furthermore, $\mathrm{X}_{0}$ and $\mathrm{X}_{0}^{\prime}$ are smooth normal crossing divisors in $\mathbb{P}_{\hat{\Delta}}$ and $\mathbb{P}_{\hat{\Delta}}^{\prime}$ respectively, and $\mathrm{X}_{0}^{\prime}$ is a reduced strict normal crossing divisors in $\mathcal{X}^{\prime}$ - i.e. $\mathcal{X}^{\prime} \rightarrow \Delta$ is a semistable degeneration. Because this is a "partial" toric degeneration (i.e. $\mathrm{X}_{0} \neq \mathbb{D}_{\hat{\Delta}}$ ), these facts are not automatic. The normal crossing divisors property is checked by computations in local coordinate systems associated to the individual tetrahedra in $\operatorname{tr}\left(\mathfrak{f}_{\mathrm{b}}^{\circ}\right)$ and $\operatorname{tr}\left(\mathfrak{f}_{\mathrm{c}}^{\circ}\right)$; it holds for the triangulation described in $\S 5.2$, but not for another triangulation we considered. Also visible in these local coordinates is the fact that the equation of $\mathcal{X}^{\prime}$ takes the form $t=\prod_{i=1}^{M} f_{i}(\underline{X})(M \leq 4)$ where the $\vec{\nabla} f_{i}(\underline{X})$ are independent along intersections of the respective components. (Since these computations are both lengthy and straightforward, we omit them.)

We describe the degenerated elliptic fibration $\mathrm{X}_{0} \stackrel{\rho}{\rightarrow} \mathbb{P}_{\Delta}$, noting that as $t \rightarrow 0$ $\left(z_{0} \rightarrow 0\right), D_{\underline{a}, z_{0}} \rightarrow D_{\underline{a}, 0}=\mathbb{D}_{\Delta}$. Over $\mathbb{P}_{\Delta}$, there are 5 components (including $\bar{Y}$ ), forming an $I_{5}$ over $\mathbb{G}_{m}^{2} \backslash E^{*}$ and an $I_{6}$ over $E^{*}$. Over each $\mathbb{P}^{1} \subset \mathbb{D}_{\Delta}, \mathrm{X}_{0}$ has 11 components; while over each point in $\mathbb{S}_{\Delta}$, there are 14 . $\bar{Y}$ itself is generically a $\mathbb{P}^{1}$-bundle, whose fiber breaks into $2 \mathbb{P}^{1}$ 's $($ joined at $(u, v)=(0,0))$ over $E^{*}$ and $8 \mathbb{P}^{1}$ 's over $\mathbb{D}_{\Delta} \backslash\left\{\mathbb{S}_{\Delta} \cup \mathbb{B}_{\Delta}\right\}$, while $\rho^{-1}\left(\mathbb{B}_{\Delta}\right) \cap \bar{Y}$ is a configuration of 5 rational surfaces (in addtion to the 11 which lie over every point of $\left.\mathbb{D}_{\Delta} \backslash \mathbb{S}_{\Delta}\right)$. This description does not change for $\mathrm{X}_{0}^{\prime}$.

5.6. Middle cohomology of $\mathrm{X}_{0}$. - By Clemens-Schmid [C, Sc] we have an exact sequence of mixed Hodge structure

$$
H_{5}\left(\mathrm{X}_{0}^{\prime}\right)(-4) \rightarrow H^{3}\left(\mathrm{X}_{0}^{\prime}\right) \rightarrow H_{\text {lim }}^{3}\left(\mathrm{X}_{t}\right) \stackrel{N}{\rightarrow} H_{\text {lim }}^{3}\left(\mathrm{X}_{t}\right)(-1)
$$

since $\mathcal{X}^{\prime} \rightarrow \Delta$ is a semistable degeneration. Using the combinatorics of $\operatorname{tr}\left(\hat{\Delta}^{\circ}\right)$ one shows that $\oplus H^{4}\left(W_{i}^{\prime}\right) \rightarrow \oplus H^{4}\left(W_{i j}^{\prime}\right)$, and clearly $\oplus H^{5}\left(W_{i}^{\prime}\right)=H^{5}\left(\bar{Y}^{\prime}\right)=H_{1}\left(\bar{Y}^{\prime}\right)(-3)$ which we shall show is zero (see below). So $H^{5}\left(\mathrm{X}_{0}^{\prime}\right)$, hence $H_{5}\left(\mathrm{X}_{0}^{\prime}\right)$, is zero; writing $H_{i n v}^{3}\left(\mathrm{X}_{t}\right)$ for the mixed Hodge structure $\operatorname{ker}(T-I)=\operatorname{ker}(N) \subset H_{\text {lim }}^{3}\left(\mathrm{X}_{t}\right)$, we have

$$
H^{3}\left(\mathrm{X}_{0}^{\prime}\right) \cong H_{i n v}^{3}\left(\mathrm{X}_{t}\right)
$$

Remark 5.2. - The only possible discrepancy between $H^{3}\left(\mathrm{X}_{0}^{\prime}\right)$ and $H^{3}\left(\mathrm{X}_{0}\right)$ is in $G r_{2}^{W}$, for which we have the diagram

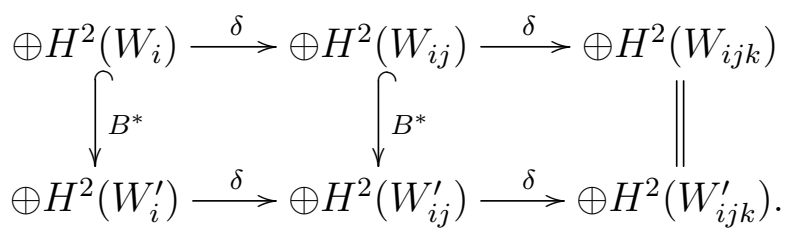

Any topological cycle $Z$ with $[Z] \in \operatorname{ker}(\delta) \subset \oplus H^{2}\left(W_{i j}\right)$ can be moved to avoid the blowup points. As a consequence, if $B^{-1}(Z)=\delta \mathfrak{Z}$ then $Z=\delta(B(\mathfrak{Z}))$, showing $H^{3}\left(\mathrm{X}_{0}\right) \hookrightarrow H^{3}\left(\mathrm{X}_{0}^{\prime}\right)$. 
Recalling that $Y_{\underline{a}}^{\prime}=W_{0}^{\prime}$, set

$$
Y_{\underline{a}}:=\bar{Y}_{\underline{a}} \cap\left(\mathbb{G}_{m}^{2} \times \mathbb{A}^{2}\right)=\bar{Y}_{\underline{a}}^{\prime} \cap\left(\mathbb{G}_{m}^{2} \times \mathbb{A}^{2}\right) \subset \bar{Y}_{\underline{a}}^{\prime} \backslash\left\{\bar{Y}_{\underline{a}}^{\prime} \cap\left(\oplus_{i \geq 1} W_{i}^{\prime}\right)\right\},
$$

the solution set of $f_{\underline{a}}(x, y)+u v=0$ with $x, y \in \mathbb{C}^{*}$ and $u, v \in \mathbb{C}$. It is a $\mathbb{G}_{m}$-bundle over $\mathbb{G}_{m}^{2} \backslash E_{\underline{a}}$ which degenerates to two affine lines meeting at $(u, v)=\underline{0}$ over $E_{\underline{a}}$. As in [DK, §5.1] we have exact sequences of mixed Hodge structure

$$
\begin{aligned}
H_{k-1}\left(E_{\underline{a}}^{*}\right)(1) & \stackrel{\left(I_{*}, 0\right)}{\rightarrow} H_{k-1}\left(\mathbb{G}_{m}^{2}\right)(1) \oplus H_{k}\left(\mathbb{G}_{m}^{2}\right) \\
& \rightarrow H_{k}\left(Y_{\underline{a}}\right) \rightarrow H_{k-2}\left(E_{\underline{a}}^{*}\right)(1) \rightarrow H_{k-2}\left(\mathbb{G}_{m}^{2}\right)(1) .
\end{aligned}
$$

Setting $k=1$ gives $H_{1}(Y)=H_{1}\left(\mathbb{G}_{m}^{2}\right) \otimes$ [pt.], which evidently maps to 0 in $H_{1}\left(\bar{Y}^{\prime}\right)$, so that $H_{1}\left(\bar{Y}^{\prime}\right)=\{0\}$.

For $k=3$, (5.11) becomes

$$
0 \rightarrow H_{2}\left(\mathbb{G}_{m}^{2}\right)(1) \stackrel{\otimes\left[S^{1}\right]}{\rightarrow} H_{3}\left(Y_{\underline{a}}\right) \stackrel{\xi}{\rightarrow} \mathcal{K}_{E}(1) \rightarrow 0 .
$$

The cycles $\left\{\mathcal{M}_{\alpha}(\varphi)\right\}_{\varphi \in \mathcal{K}_{E}}$ and $\mathcal{T}_{\alpha}$ evidently limit (with $\left.t \rightarrow 0\right)$ to cycles $\{\mathcal{M}(\varphi)\}_{\varphi \in \mathcal{K}_{E}}$ and $\mathcal{T}$ on $Y_{a}$, with the $S^{1}$ on the $\mathbb{G}_{m}$-fibers replacing $\alpha$. Clearly $[\mathcal{T}]=\operatorname{im}\left(\otimes\left[S^{1}\right]\right)$, and $\operatorname{span}\{[\overline{\mathcal{M}}(\varphi)]\}$ maps isomorphically to $\mathcal{K}_{E}(1)$ (cf. the construction of the rightinverse $^{(7)}$ " $M$ " to $\xi$ in $[\mathrm{DK}, \S 5.1]$ So as a $\mathbb{Q}$-vector space, $H_{3}\left(Y_{\underline{a}}\right) \cong \Phi_{E} \oplus\langle\mathcal{T}\rangle$; as a mixed Hodge structure, $H_{3}\left(Y_{\underline{a}}\right)(-3)$ is an extension of $\mathcal{K}_{E}(-2)$ (which has type $(2,1)+(1,2)+(1,1))$ by a $\mathbb{Q}(0)($ spanned by $\mathcal{T})$.

Now consider the composite morphism

$$
\begin{aligned}
\Theta^{\prime}: H_{3}\left(Y_{\underline{a}}\right)(-3) & \cong H^{3}\left(\bar{Y}^{\prime}, \bar{Y}^{\prime} \backslash Y\right) \rightarrow H^{3}\left(W_{0}^{\prime}, \cup W_{0 i}^{\prime}\right) \\
& =H^{3}\left(\mathrm{X}_{0}^{\prime}, \cup_{i \geq 1} W_{i}^{\prime}\right) \rightarrow H^{3}\left(\mathrm{X}_{0}^{\prime}\right)
\end{aligned}
$$

of mixed Hodge structure (one defines $\Theta$ similarly). On the level of closed chains, $\Theta^{\prime}$ is induced by

$$
\begin{aligned}
\tilde{\Theta}^{\prime}: Z_{3}^{\text {top }}(Y) & \rightarrow \operatorname{ker}\left\{Z_{3}^{\text {top }}\left(\bar{Y}^{\prime}\right)_{\#} \rightarrow \oplus_{i} Z_{i}^{\text {top }}\left(W_{0 i}^{\prime}\right)\right\} \\
& \hookrightarrow \operatorname{ker}\left\{\delta: \oplus_{i} Z_{\text {top }}^{3}\left(W_{i}^{\prime}\right)_{\#} \rightarrow \oplus_{i, j} Z_{\text {top }}^{3}\left(W_{i j}^{\prime}\right)\right\} \\
& \rightarrow \operatorname{ker}\left\{\mathbb{D}: \oplus_{I} Z_{\text {top }}^{3-|I|}\left(W_{I}^{\prime}\right)_{\#} \rightarrow \oplus_{J} Z_{\text {top }}^{4-|J|}\left(W_{J}^{\prime}\right)\right\}
\end{aligned}
$$

where \# denotes intersection conditions and $\mathbb{D}$ is the total differential for the complex computing $H^{*}\left(\mathrm{X}_{0}^{\prime}\right)$. The main point here is that the Clemens retraction map $\mathfrak{r}$ : $H^{3}\left(\mathrm{X}_{0}^{\prime}\right) \rightarrow H^{3}\left(\mathrm{X}_{t}\right)$ is given (on $\operatorname{ker}(\delta)$, hence $\operatorname{im}\left(\tilde{\Theta}^{\prime}\right)$ ) by simple preimage under $\mathfrak{r}$ :

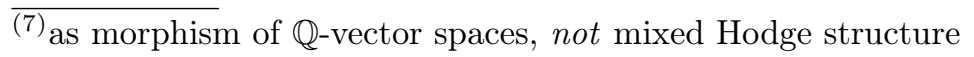


$\mathrm{X}_{t} \rightarrow \mathrm{X}_{0}^{\prime}$. Since this obviously sends $\mathcal{T} \mapsto \mathcal{T}_{\alpha}$ and $\mathcal{M}(\varphi) \mapsto \mathcal{M}_{\alpha}(\varphi)$, the composite MHS morphism

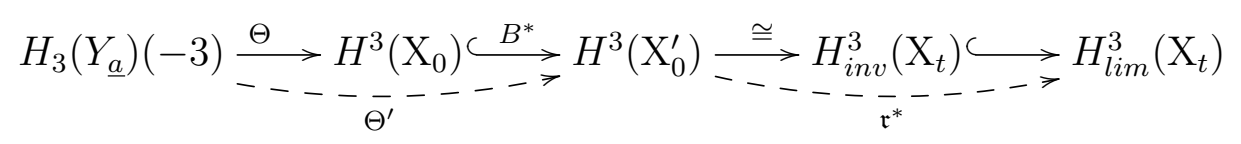

sends $[\mathcal{T}] \mapsto\left[\mathcal{T}_{\alpha}\right]$ and $[\mathcal{M}(\varphi)] \mapsto\left[\mathcal{M}_{\alpha}(\varphi)\right]$. Since these classes remain independent in $H^{3}\left(\mathrm{X}_{t}\right),{ }^{(8)} \Theta$ and $\Theta^{\prime}$ are injective. Consequently $H_{\text {lim }}^{3}\left(\mathrm{X}_{t}\right)$ has $I^{0,0} \supseteq I^{0,0}\left(H_{3}(Y)(-3)\right)$ of rank at least $1, I^{1,1} \supseteq N^{+}\left(I^{0,0}\right) \oplus I^{1,1}\left(H_{3}(Y)(-3)\right)$ of rank at least $1+(r-3)=r-2$, and $I^{2,1} \cong I^{1,2} \supset I^{1,2}\left(H_{3}(Y)(-3)\right)$ of rank at least 1 . The only possible limiting mixed Hodge structure type, given that $H_{\text {lim }}^{3}\left(\mathrm{X}_{t}\right)$ has $G r_{F}^{i}$ ranks $1, r-1, r-1,1$, is

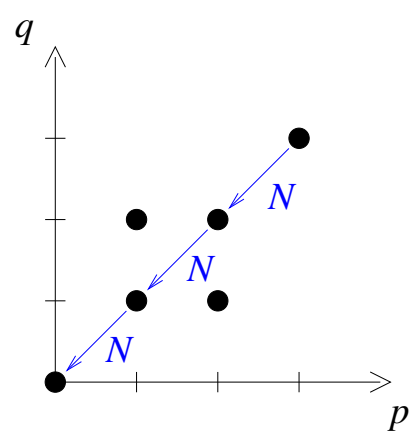

$$
\begin{array}{r}
1=\operatorname{rk}\left(I^{0,0}\right)=\operatorname{rk}\left(I^{2,1}\right)=\operatorname{rk}\left(I^{1,2}\right)=\operatorname{rk}\left(I^{3,3}\right) \\
r-2=\operatorname{rk}\left(I^{1,1}\right)=\operatorname{rk}\left(I^{2,2}\right) \\
\text { (all others zero) }
\end{array}
$$

This implies at once that (the image of) $H_{3}\left(Y_{\underline{a}}\right)(-3)$ is all of $\operatorname{ker}(N)=H_{i n v}^{3}\left(\mathrm{X}_{t}\right)$, and so $\Theta, \Theta^{\prime}$, and $B^{*}$ are all isomorphisms:

Theorem 5.3. - We have isomorphisms of $\mathbb{Q}$-variation of mixed Hodge structure

$$
H_{3}\left(Y_{\underline{a}}\right)(-3) \cong H^{3}\left(\mathrm{X}_{\underline{a}, 0}\right) \cong H^{3}\left(\mathrm{X}_{\underline{a}, 0}^{\prime}\right) \cong H_{i n v}^{3}\left(\mathrm{X}_{\underline{a}, t}\right) \text {. }
$$

5.7. Monodromy and asymptotics of periods. - We begin by addressing the nature of the limiting periods (i.e., by Theorem 5.3, periods on $Y_{\underline{a}}$ ). Set

$$
\begin{aligned}
\eta_{\underline{a}} & :=\frac{1}{(2 \pi \mathbf{i})^{3}} \operatorname{Res}_{Y_{\underline{a}}}\left(\frac{d x / x \wedge d y / y \wedge d u \wedge d v}{f_{\underline{a}}(x, y)+u v}\right) \\
& =\left.\frac{1}{(2 \pi \mathbf{i})^{3}} \frac{d x}{x} \wedge \frac{d y}{y} \wedge \frac{d u}{u}\right|_{Y_{\underline{a}}} \in \Omega^{3}\left(Y_{\underline{a}}\right) .
\end{aligned}
$$

Write

$$
R\{x, y\}:=\log (x) \frac{d y}{y}-2 \pi \mathbf{i} \log (y) \delta_{T_{x}}
$$

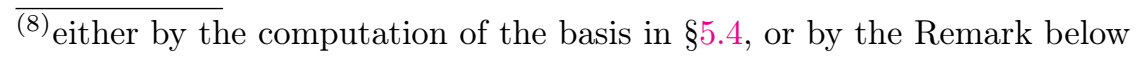


for the 1-current on $\mathbb{G}_{m}^{2}$, where $\log (x)$ is the (discontinuous) branch with argument in $(-\pi, \pi)$, and $T_{x}=x^{-1}\left(\mathbb{R}_{-}\right)$(with $\mathbb{R}_{-}$oriented from $-\infty$ to 0 ). For any invariant 3 -cycle $\kappa$,

$$
\lim _{t \rightarrow 0} \int_{\kappa} \Omega_{\underline{a}, t}=\int_{\kappa} \eta_{\underline{a}}
$$

which for $\kappa=\mathcal{T}$ is 1 and for $\kappa=\mathcal{M}(\varphi)$ is (according to [DK, §5.1])

$$
\frac{1}{(2 \pi \mathbf{i})^{2}} \int_{\Gamma_{\varphi}} \frac{d x}{x} \wedge \frac{d y}{y}=\left.\frac{1}{(2 \pi \mathbf{i})^{2}} \int_{\varphi} R\{x, y\}\right|_{E_{\underline{a}}^{*}}=: \frac{1}{2 \pi \mathbf{i}} R_{\varphi}(\underline{a}) .
$$

These "regulator periods" were computed in [DK, §5.2] for a specific choice ${ }^{(9)}$ of $\nu-2$ $\left\{\varphi_{0}^{(i)}\right\}$ and with only those $\left\{a_{j}\right\}$ attached to vertices nonzero. If $\Delta$ has $\nu=r$ (no interior integral points on edges), then these $\left\{\varphi_{0}^{(i)}\right\}$ are "enough" (we need $r-2$ ) and no $a_{j}$ are set equal to 0 .

In that case, and with that choice of basis - to both of which we shall restrict for the moment - we get an alternate proof of the independence of the $r$ invariant cycles $\left\{\mathcal{M}_{\alpha}\left(\varphi_{1}\right),\left\{\mathcal{M}_{\alpha}\left(\varphi_{0}^{(i)}\right)\right\}_{i=1}^{r-2}, \tau_{\alpha}\right\}$ in $H_{(\text {lim })}^{3}\left(\mathrm{X}_{t}\right)$, which is simpler than constructing the full basis of $H^{3}\left(\mathrm{X}_{t}\right)$. Namely, observe (cf. [DK, §5.2]) that

$$
\left\{\begin{array}{l}
R_{\varphi_{0}^{(i)}}(\underline{a}) \sim \log \left(z_{i}\right) \quad(i=1, \ldots, r-2) \\
R_{\varphi_{1}}(\underline{a}) \sim \frac{1}{2 \pi \mathbf{i}} Q\left(\log \left(z_{1}\right), \ldots, \log \left(z_{r-2}\right)\right) \quad\left(\begin{array}{c}
Q \text { quadratic, } \\
\text { with } \mathbb{Q} \text {-coeffs. }
\end{array}\right)
\end{array}\right.
$$

are independent functions as $\underline{a}$ varies; therefore, so are the $\int_{\mathcal{M}_{\alpha}(\varphi)} \Omega_{\underline{a}, t}$ and $\int_{\tau_{\alpha}} \Omega_{\underline{a}, t}$, and independence of the cycle classes follows.

From (5.15) we also obtain information on the asymptotics of periods of $H^{3}\left(\mathrm{X}_{F}\right)$ which will be key for defining the mirror map in $\S 6.4$. Writing $T_{j}$ for the monodromy about $z_{j}=0$ (and $N_{j}=\log \left(T_{j}\right)$ ), $\mathcal{T}_{\alpha}$ is the cycle whose "Tube" (for all $\left|z_{i}\right| \ll \epsilon$ ) is $\cap_{i=1}^{r-2}\left\{\left|z_{i}\right|=\epsilon\right\}$, hence is invariant by all $T_{j}$. (In particular, we have $\lim _{t \rightarrow 0} \int_{\mathcal{T}_{\alpha}} \Omega_{F}=1$.) Putting

we define normalized B-model periods by

$$
\tilde{\Omega}_{F}:=\frac{\Omega_{F}}{\int_{\tau_{\alpha}} \Omega_{F}},
$$

$$
\Pi_{\gamma}^{\mathrm{B}}(\underline{z}):=\int_{\gamma} \tilde{\Omega}_{F} \quad\left(\gamma \in H_{3}\left(\mathrm{X}_{F}, \mathbb{Z}\right)\right)
$$

Obviously $\Pi_{\mathcal{T}_{\alpha}}^{\mathrm{B}}$ is identically 1 whilst (using (5.14) and (5.15))

$$
\Pi_{\mathcal{M}_{\alpha}^{\mathrm{B}}\left(\varphi_{0}^{(i)}\right)} \sim \frac{1}{2 \pi \mathbf{i}} \log \left(z_{i}\right) \quad(i>0) .
$$

$\overline{{ }^{(9)} \text { cf. p. } 487}$ of [DK, $\left.\S 5.2\right]$, where these "distinguished vanishing cycles" are denoted $\varphi_{0}^{[i]}$. Also see [BKV, §4.1-2] for a brief introduction to regulator currents in the context of Feynman integrals. 
Now when we take all $\left\{a_{i}\right\}_{i>0}$, hence all $\left\{z_{i}\right\}_{i>0}$, to zero, the equation for $\mathrm{X}$ becomes

$$
\mathrm{b} u^{3}+\mathrm{c} v^{2}+\mathrm{a} u v+a_{0}=0 .
$$

That is, we are left with an isotrivial family of elliptic curves $\cong: \mathcal{E}_{z_{0}}$ over $\mathbb{G}_{m}^{2}$ (as $\rho^{-1} E$ and $\rho^{-1} D$ have both collapsed to $\rho^{-1} \mathbb{D}$ ), with $\rho^{-1} \mathbb{D}$ consisting of all 3 -fold components of $\mathbb{D}_{\hat{\Delta}}$ associated to points of $\hat{\Delta}_{\mathbb{Z}}^{\circ}$ dual to an edge or vertex of $\Delta \times(-1,-1)$. Clearly $\mathcal{T}_{\alpha}$ and $\mathcal{T}_{\beta}$ remain in the cohomology of this singular 3 -fold, so $\mathcal{T}_{\beta}$ is invariant under all $\left\{T_{j}\right\}_{j>0}$. Taking $\varpi_{z_{0}} \in \Omega^{1}\left(\mathcal{E}_{z_{0}}\right)$ to be normalized so that $\int_{\alpha} \varpi_{z_{0}}=1$, the limiting period

$$
\Pi_{\mathcal{T}_{\beta}}^{\mathrm{B}}\left(z_{0}, \underline{0}\right)=\frac{1}{(2 \pi \mathbf{i})^{2}} \int_{\mathcal{T}_{\beta}} \frac{d x}{x} \wedge \frac{d y}{y} \wedge \varpi_{z_{0}}=\int_{\beta} \varpi_{z_{0}}
$$

is asymptotic to $\frac{5}{2 \pi \mathbf{i}} \log t$ ( $\operatorname{since} \mathcal{E}_{z_{0}}$ limits to an $I_{5}$ at $t=0$ ); therefore

$$
\Pi_{\mathcal{T}_{\beta}}^{\mathrm{B}} \sim \frac{1}{2 \pi \mathbf{i}} \log \left(z_{0}\right)
$$

Remark that by (5.16), the $\left\{\mathcal{M}_{\alpha}\left(\varphi_{0}^{(i)}\right)\right\}_{i \neq j}$ are invariant under $T_{j}$ for $j>0$; equivalently, the membrane in $\mathbb{G}_{m}^{2} \backslash D^{*}$ bounding on each $\varphi_{0}^{(i)} \subset E^{*}(i \neq j)$ behaves well in the $z_{j} \rightarrow 0$ limit (under which $E$ and $D$ become nodal rational curves in the same linear system). Symmetrically, there are ${ }^{\prime} \varphi_{0}^{(i)} \subset D^{*}$ with the same properties. So for $j>0$,

$$
\operatorname{ker}\left(T_{j}-I\right)=\operatorname{ker}\left(N_{j}\right)=\left\langle\mathcal{T}_{\alpha}, \mathcal{T}_{\beta},\left\{\mathcal{M}_{\alpha}\left(\varphi_{0}^{(i)}\right)\right\}_{i \neq j},\left\{\mathcal{M}_{\beta}\left({ }^{\prime} \varphi_{0}^{(i)}\right)\right\}_{i \neq j}\right\rangle,
$$

while as previously remarked $\operatorname{ker}\left(N_{0}\right)=\left\langle\mathcal{T}_{\alpha},\left\{\mathcal{M}_{\alpha}\left(\varphi_{0}^{(i)}\right)\right\}_{i=1}^{r-2}, \mathcal{M}_{\alpha}\left(\varphi_{1}\right)\right\rangle$. Combining this with the fact that $\mathcal{M}_{\beta}\left(\varphi_{1}\right)$ is the only cycle in our basis pairing nontrivially with $\mathcal{T}_{\alpha}$ (e.g., consider the above $z_{1}, \ldots, z_{r-2} \rightarrow 0$ limit), we can compute a basis for $W_{\bullet}^{0}:=$ $W\left(N_{0}\right)_{\bullet}$. Writing $\left\{\psi_{I}\right\}_{i=1}^{r-3}$ for a basis of $\operatorname{ker}\left\{\mathcal{K}_{E} \rightarrow H_{1}(E)\right\}$, so that $\mathcal{K}_{E}=\left\langle\left\{\psi_{i}\right\}, \varphi_{0}^{(1)}\right\rangle$, we have

$$
\begin{gathered}
W_{0}^{0}=\left\langle\mathcal{T}_{\alpha}\right\rangle, \quad W_{2}^{0}=W_{0}^{0}+\left\langle\left\{\mathcal{M}_{\alpha}\left(\psi_{i}\right)\right\}_{i=1}^{r-3}, \mathcal{T}_{\beta}\right\rangle \\
W_{3}^{0}=W_{2}^{0}+\left\langle\mathcal{M}_{\alpha}\left(\varphi_{0}^{(1)}\right), \mathcal{M}_{\alpha}\left(\varphi_{1}\right)\right\rangle \\
W_{4}^{0}=W_{3}^{0}+\left\langle\left\{\mathcal{M}_{\beta}\left(\varphi_{0}^{(i)}\right)\right\}_{i=1}^{r-2}\right\rangle, \quad W_{6}^{0}=W_{4}^{0}+\left\langle\mathcal{M}_{\beta}\left(\varphi_{1}\right)\right\rangle .
\end{gathered}
$$

For the (Hodge-Tate) limit at $\underline{z}=\underline{0}$, we have $\left(\right.$ for $W_{\bullet}=W\left(N_{0}+\cdots+N_{r-2}\right) \bullet$ )

$$
\begin{gathered}
W_{0}=\left\langle\mathcal{T}_{\alpha}\right\rangle, \quad W_{2}=W_{0}+\left\langle\mathcal{T}_{\beta},\left\{\mathcal{M}_{\alpha}\left(\varphi_{0}^{(i)}\right)\right\}_{i=1}^{r-2}\right\rangle, \\
W_{4}=W_{2}+\left\langle\left\{\mathcal{M}_{\beta}\left({ }^{\prime} \varphi_{0}^{(i)}\right)\right\}_{i=1}^{r-2}, \mathcal{M}_{\alpha}\left(\varphi_{1}\right)\right\rangle, \quad W_{6}=W_{4}+\left\langle\mathcal{M}_{\beta}\left({ }^{\prime} \varphi_{1}\right)\right\rangle .
\end{gathered}
$$

The other $W\left(N_{j}\right)$.'s are more difficult and will be computed via the A-model in $\S 6.3$.

We will say more about the specialization to the sunset case, where

$$
f_{\underline{a}}=a_{0}+a_{1} x+a_{2} y+a_{3} x^{-1} y+a_{4} x^{-1}+a_{5} y^{-1}+a_{6} x y^{-1},
$$


in the next section, but some preliminary remarks are in order. Writing $\hat{\varphi}_{0}^{(i)}$ for the vanishing cycle in $H_{1}\left(E_{\underline{a}}^{*}\right)$ for $a_{i} \rightarrow 0$, we have (with indices modulo 6) $\varphi_{0}^{(j)}=-\hat{\varphi}_{0}^{(j)}+$ $\hat{\varphi}_{0}^{(j-1)}+\hat{\varphi}_{0}^{(j+1)}$, which all map to the same cycle $\varphi_{0} \in H_{1}\left(E_{\underline{a}}\right)$, and

$$
z_{j}=\frac{a_{j+1} a_{j-1}}{a_{j} a_{0}}, \quad j=1, \ldots, 6 .
$$

The apparent obstacle here is that although $r=r^{\circ}=\nu=6$, the 4-dimensional cone spanned by the vectors $\underline{\ell}^{(j)}$ of $[\mathrm{DK}]\left(\ell_{j}^{(j)}=-1, \ell_{j-1}^{(j)}=\ell_{j+1}^{(j)}=1, \ell_{\text {other }}^{(j)}=0\right)$ is not simplicial. Hence $z_{1}$ thru $z_{4}$ do not suffice to parametrize the resulting singular local parameter 4-space, and $\varphi_{1},\left\{\varphi_{0}^{(j)}\right\}_{j=1}^{4}$ span $\mathcal{K}_{E}$ rationally but not integrally. (We need all $6\left\{z_{i}\right\}$ and all $6\left\{\varphi_{0}^{(j)}\right\}$, and the relations on the $\left\{z_{i}\right\}$ produce the singularity.) Writing $R(m) \subseteq \mathbb{Z}^{6}$ for the set of "relations vectors" $\left(\ell_{1}, \ldots, \ell_{6}\right)$ with $\sum \ell_{j}=m \in \mathbb{N}$, $\sum \ell_{j} \underline{m}^{(j)}=\underline{0}$, we have $(j=1, \ldots, 6)$

$$
\begin{aligned}
\lim _{z_{0} \rightarrow 0} 2 \pi \mathbf{i} \cdot \Pi_{\mathcal{M}_{\alpha}\left(\varphi_{0}^{(j)}\right.}^{\mathrm{B}} & =R_{\varphi_{0}^{(i)}}(\underline{a})=\log \left(z_{j}\right)+H(\underline{a}) \\
& =\log \left(z_{j}\right)+\sum_{m \geq 1} \frac{1}{m} \sum_{\underline{\ell \in R(m)}} \frac{m !}{\ell_{1} ! \cdots \ell_{6} !} \cdot \frac{a_{1}^{\ell_{1}} \cdots a_{6}^{\ell_{6}}}{\left(-a_{0}\right)^{m}}
\end{aligned}
$$

for the limiting periods (cf. [DK, eqn (5.4)]).

Upon specializing to the "Feynman locus" where

$$
f_{\underline{a}}=1-s \phi_{\ominus}, \quad \phi_{\ominus}:=\left(-\xi_{1}^{2} x-\xi_{2}^{2} y+\xi_{3}^{2}\right)\left(1-x^{-1}-y^{-1}\right)
$$

a small miracle occurs. The resulting substitutions $\underline{a}=\left(\xi_{1}, \xi_{2}, \xi_{3}, s\right)$ yield

$$
z_{1}=-\frac{\xi_{2}^{2} s}{a_{0}}=z_{4}, \quad z_{2}=-\frac{\xi_{1}^{2} s}{a_{0}}=z_{5}, \quad z_{3}=-\frac{\xi_{3}^{2} s}{a_{0}}=z_{6}
$$

where $a_{0}=1-s \sum \xi_{i}^{2}$, and $R_{0}^{(j)}:=R_{\varphi_{0}^{(i)}}(\underline{a})=$

$$
\log \left(z_{j}\right)+\sum_{m \geq 1} \frac{1}{m} \sum_{\underline{\ell \in R(\underline{m})}} \frac{(-1)^{\ell_{3}+\ell_{6}} m !}{\ell_{1} ! \cdots \ell_{6} !} z_{1}^{\ell_{2}+\ell_{3}} z_{2}^{\ell_{1}+\ell_{6}} z_{3}^{\ell_{4}+\ell_{5}}
$$

which will be considered as a function of $\left(z_{1}, z_{2}, z_{3}\right)$. In particular, we have

$$
R_{0}^{(1)}=R_{0}^{(4)}, \quad R_{0}^{(2)}=R_{0}^{(5)}, \quad R_{0}^{(3)}=R_{0}^{(6)} ;
$$

in effect, the specialization has replaced a singular 4-fold local parameter space by a smooth 3-dimensional slice. From the standpoint of periods (of $R\{x, y\}$ on $E^{*}$, or $\eta$ on $Y)$, the class

$$
-\varphi_{0}^{(1)}+\varphi_{0}^{(4)}=\varphi_{0}^{(2)}-\varphi_{0}^{(5)}=-\varphi_{0}^{(3)}+\varphi_{0}^{(4)}
$$

in $\mathcal{K}_{E}$ is now "trivial", and the quotient $\overline{\mathcal{K}_{E}}$ of $\mathcal{K}_{E}$ by (5.22) is integrally spanned by $\varphi_{0}^{(1)}, \varphi_{0}^{(2)}, \varphi_{0}^{(3)}$. Recalling that $\operatorname{ker}\left(N_{0}\right) \subset H_{\text {lim }}^{3}(\mathrm{X})$ is an extension

$$
\mathbb{Q}(0) \rightarrow \operatorname{ker}\left(N_{0}\right) \rightarrow \mathcal{K}_{E}(-2),
$$


the immediate consequence is that the $\mathbb{Q}(-1) \subset \mathcal{K}_{E}(-2)$ spanned by (5.22) lifts to a $\mathbb{Q}(-1) \subset \operatorname{ker}\left(N_{0}\right)$. It is the quotient $\overline{\operatorname{ker}\left(N_{0}\right)}$ by this constant sub-variation of mixed Hodge structure which we will be interested in when comparing with the A-model.

It will be useful in the sequel to denote by $\mathcal{V}_{\mathrm{B}}$ the $\mathbb{Z}$-variation of Hodge structure $H^{3}\left(\mathrm{X}_{F}\right)$ considered over a product of punctured disks with parameters $z_{0}, \ldots, z_{r-2}$.

\section{A-model}

We now turn to the (integral) variation of Hodge structure arising from the quantum product on $H^{\text {even }}\left(\mathrm{X}^{\circ}\right)$, where $\mathrm{X}^{\circ} \subset \mathbb{P}_{\hat{\Delta}^{\circ}}$ is the Batyrev mirror of $\mathrm{X}$. Its equivalence to the B-model variation of Hodge structure $H^{3}(\mathrm{X})$ allows us to compute all monodromies of the latter (about the hyperplanes $\left\{z_{i}=0\right\}$ ) and relate its $z_{0} \rightarrow 0$ limiting mixed Hodge structure to local Gromov-Witten data for $\mathbb{P}_{\Delta^{\circ}}$. In order to make use of the computations in [DK, §5], we shall work under the assumption that $\nu=r$ (so that $(\partial \Delta)_{\mathbb{Z}}$ consists of vertices).

6.1. Elliptic fibration and even cohomology. - As in the B-model case, triangulating $\partial \hat{\Delta}$ produces a resolution of singularities $\mathbb{P}_{\hat{\Delta}^{\circ}} \rightarrow \check{\mathbb{P}}_{\hat{\Delta}^{\circ}}$. The desired triangulation is achieved by:

- inserting the $\frac{1}{2}$-planes $\mathbb{H}_{\underline{w}}\left(\underline{w} \in(\partial \Delta)_{\mathbb{Z}}\right)$ as in Step 1 of $\S 5.2$, which subdivides the 2 -face $\Delta \times(-1,-1)$ and each of the facets

$$
\begin{aligned}
& \mathfrak{f}_{1}=\operatorname{hull}\{\Delta \times(-1,-1),(0,0,-1,1)\}, \\
& \mathfrak{f}_{2}=\operatorname{hull}\{\Delta \times(-1,-1),(0,0,2,-1)\}
\end{aligned}
$$

into $r$ pieces; and

- further subdividing the facet-pieces by inserting 2-planes through the edges of $\Delta \times(-1,-1)$ and $(0,0,1,-1),(0,0,0,-1)$, resp. $(0,0,-1,0)$.

The first step guarantees a morphism $\mathbb{P}_{\hat{\Delta}^{\circ}} \stackrel{\mathcal{P}^{\circ}}{\rightarrow} \mathbb{P}_{\Delta^{\circ}}$, with generic fiber $\mathbb{P}_{W}$. Its restriction to an anticanonical (Calabi-Yau) hypersurface $\mathrm{X}^{\circ} \stackrel{\imath}{\subset} \mathbb{P}_{\hat{\Delta}^{\circ}}$, cut out by a generic Laurent polynomial (with Newton polytope $\hat{\Delta}^{\circ}$ ), produces a Weierstrass elliptic fibration $\rho^{\circ}$ : $\mathrm{X}^{\circ} \rightarrow \mathbb{P}_{\Delta^{\circ}}$. The discriminant locus of $\rho^{\circ}$ (over which the fiber is $I_{1}$ ) is a higher-genus curve meeting $\mathbb{D}_{\Delta^{\circ}}$ properly; in particular, $\left(\rho^{\circ}\right)^{-1}$ of components of $\mathbb{D}_{\Delta^{\circ}}$ (and of their intersections) are smooth.

Let $D_{0} \cong \mathbb{P}_{\Delta^{\circ}}$ denote the (zero-)section of $\rho^{\circ}$ given by intersecting $\mathrm{X}^{\circ}$ with the component of $\mathbb{D}_{\hat{\Delta}^{\circ}}$ dual to $(0,0,-1,-1) \in(\partial \hat{\Delta})_{\mathbb{Z}}$. Writing $\mathbb{D}_{\Delta^{\circ}}=\cup_{i=1}^{r} C_{i}$ (with the counterclockwise ordering), the divisors $D_{i}:=\left(\rho^{\circ}\right)^{-1}\left(C_{i}\right)$ are the intersections with $\mathrm{X}^{\circ}$ 
of components dual to the $\left\{\underline{v}_{i} \times(-1,-1)\right\}$ (where $\left\{\underline{v}_{i}\right\}$ are the vertices of $\Delta$ ).

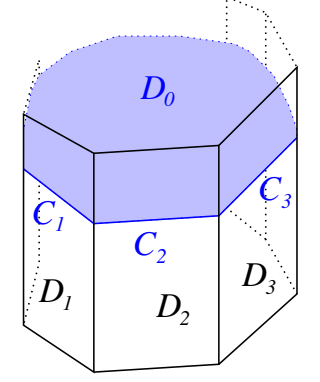

(In the sequel, $C_{i}$ will mean $D_{0} \cap D_{i} \subset \mathrm{X}^{\circ}$.) There are five more components of $\mathbb{D}_{\hat{\Delta}^{\circ}}$ : those dual to $(0,0,1,-1),(0,0,0,-1)$, and $(0,0,-1,0)$ do not meet $\mathrm{X}^{\circ}$; and we denote by $D^{\prime}, D^{\prime \prime}$ the intersections with $\mathrm{X}^{\circ}$ of those dual to $(0,0,2,-1)$ resp. $(0,0,-1,1)$.

The divisors of the toric coordinates $\left\{X_{i}\right\}_{i=1}^{4}$ restricted to $\mathrm{X}^{\circ}$ are then given by

$$
\left\{\begin{array}{l}
\left(X_{1}\right)=\sum_{i=1}^{r} v_{i}^{(1)} D_{i}, \quad\left(X_{2}\right)=\sum_{i=1}^{r} v_{i}^{(2)} D_{i}, \\
\left(X_{3}\right)=2 D^{\prime}-D^{\prime \prime}-\sum_{i=0}^{r} D_{i}, \quad\left(X_{4}\right)=D^{\prime \prime}-D^{\prime}-\sum_{i=0}^{r} D_{i}
\end{array}\right.
$$

so that in $C H^{1}\left(\mathrm{X}^{\circ}\right) \cong H^{2}\left(\mathrm{X}^{\circ}, \mathbb{Z}\right)$ we have $D^{\prime} \equiv 2 \sum_{i=0}^{r} D_{i}, D^{\prime \prime} \equiv 3 \sum_{i=0}^{r} D_{i}$, and $D_{r-1}, D_{r} \in \operatorname{span}\left\langle\left\{D_{i}\right\}_{i=1}^{r-2}\right\rangle$. Now $D^{\prime} \cap D_{0}$ and $D^{\prime \prime} \cap D_{0}$ are empty (as the corresponding faces of $\hat{\Delta}^{\circ}$ meet in vertices), and so in $C H^{2}\left(\mathrm{X}^{\circ}\right)\left(\text { hence } H^{4}\left(\mathrm{X}^{\circ}, \mathbb{Z}\right)\right)^{(10)}$

$$
D_{0} \cdot D_{0} \equiv-\sum_{i=1}^{r} D_{0} \cdot D_{i}=-\sum_{i=1}^{r} C_{i} \equiv-E^{\circ}
$$

where $E^{\circ}$ is a general anticanonical (elliptic) curve in $D_{0} \cong \mathbb{P}_{\Delta^{\circ}}$. Writing $d_{i}$ for $\ell\left(i^{\text {th }}\right.$ edge of $\left.\partial \Delta^{\circ}\right)$, so that $r^{\circ}=\sum_{i=1}^{r} d_{i}$, we therefore have

$$
\left(D_{0} \cdot C_{i}\right)=\left(D_{0} \cdot D_{0} \cdot D_{i}\right)=-\left(E^{\circ} \cdot D_{i}\right)=-\left(E^{\circ} \cdot C_{i}\right)_{D_{0}}=-d_{i}
$$

for $i=1, \ldots, r$.

From the first line of (6.1), we also have $C_{r-1}, C_{r} \in \operatorname{span}\left\langle\left\{C_{i}\right\}_{i=1}^{r-2}\right\rangle$ so that $\left\{C_{i}\right\}_{i=1}^{r-2}$ span $H^{2}\left(D_{0}\right)$, and $\left[\left(C_{i} \cdot C_{j}\right)_{D_{0}}\right]_{i, j=1}^{r-2}=\left[\left(C_{i} \cdot D_{j}\right)_{\mathrm{X}^{\circ}}\right]_{i, j=1}^{r-2}$ is nondegenerate. Since a general fiber $C_{0}$ of $\rho^{\circ}$ satisfies $\left(C_{0} \cdot D_{0}\right)=1$ and $\left(C_{0} \cdot D_{i}\right)=0(i>0),\left[\left(C_{i} \cdot D_{j}\right)_{\mathrm{X}^{\circ}}\right]_{i, j=0}^{r-2}$ is in fact nondegenerate. Using that $H^{1,1}\left(\mathrm{X}^{\circ}\right)=h^{2,1}(\mathrm{X})=r-1$, it follows that a basis for

$$
V=H^{\text {even }}\left(\mathrm{X}^{\circ}, \mathbb{C}\right)=\oplus_{k=0}^{3} H^{k, k}\left(\mathrm{X}^{\circ}\right)
$$

is given by $\left\{\mathrm{X}^{\circ} ; D_{0}, \ldots, D_{r-2} ; C_{0}, \ldots, C_{r-2} ; p\right\}$ where $p \in \mathrm{X}^{\circ}$ is a point. Write

$$
J_{j}=\sum_{k=0}^{r-2} \alpha_{j}^{k} D_{k} \quad\left(\alpha_{j}^{k} \in \mathbb{Q} ; j=0, \ldots, r-2\right)
$$

$\overline{{ }^{(10)} \text { We shall use }}$ and often nothing as cup product on $H^{\text {even }}$. 
for the basis of $H^{2}\left(\mathrm{X}^{\circ}, \mathbb{Q}\right)$ Poincaré-dual to the $\left\{C_{j}\right\}_{j=0}^{r-2} \subset H^{4}\left(\mathrm{X}^{\circ}, \mathbb{Q}\right)$.

Clearly all the $\alpha_{i}^{0}=0$ for $i>0$, so using (6.3) we find that

$$
\left\{\begin{array}{l}
J_{0}=D_{0}+\left(\pi^{\circ}\right)^{-1} E^{\circ}=D_{0}+D_{1}+\cdots+D_{r}=D_{0}+\sum_{i=1}^{r-2} \alpha_{0}^{i} D_{i} \\
D_{0}=J_{0}-\sum_{i=1}^{r-2} d_{i} J_{i}
\end{array}\right.
$$

hence (by (6.2))

$$
J_{0}^{2}=r^{\circ} C_{0}+C_{1}+\cdots+C_{r}=r^{\circ} C_{0}+\sum_{i=1}^{r-2} \alpha_{0}^{i} C_{i} .
$$

For the triple-products, we evidently have $J_{0}^{3}=r^{\circ}$ (dropping the class of the point " $p$ "), and $J_{i} J_{j} J_{k}=0$ if $i, j, k>0$. For $j>0$ we find (by (6.6)

$$
J_{0}^{2} J_{j}=\sum_{i=1}^{r-2} \alpha_{0}^{i}\left(C_{i} \cdot J_{j}\right)=\alpha_{0}^{j},
$$

while for $i, j>0$

$$
J_{0} J_{i} J_{j}=\sum_{k=1}^{r-2} \alpha_{j}^{k} J_{0} J_{i} D_{k}=\sum_{k} \alpha_{j}^{k} D_{0} J_{i} D_{k}=\sum_{k} \alpha_{j}^{k}\left(J_{i} \cdot C_{k}\right)=\alpha_{j}^{i} .
$$

In particular, $\left[\alpha_{j}^{i}\right]_{i, j=1}^{r-2}$ is symmetric, which reflects the fact that it is the inverse of $\left[\left(C_{i} \cdot C_{j}\right)_{D_{0}}\right]_{i, j=1}^{r-2}$ (which can be computed from the $\ell$-vectors of $[\mathrm{DK}, \S 5]$ ).

From (6.7) and (6.8) we also have

$$
J_{i} J_{j}=\alpha_{j}^{i} C_{0} \quad \text { and } \quad J_{0} J_{j}=\sum_{i=0}^{r-2} \alpha_{i}^{j} C_{i} .
$$

Since $D_{0} \cdot J_{0}=-E^{\circ}+E^{\circ}=0$ by (6.2) and (6.5), using (6.3) and (6.9) to evaluate $(0=) D_{0} \cdot J_{0} \cdot J_{j}$ yields the intriguing relations

$$
\left\{\begin{array}{l}
\alpha_{0}^{j}=\sum_{i=1}^{r-2} d_{i} \alpha_{j}^{i} \quad(j=1, \ldots, r-2) \\
r^{\circ}=\sum_{i=1}^{r-2} d_{i} \alpha_{0}^{i}
\end{array}\right.
$$

For the sequel we set $\tilde{\alpha}_{j}^{i}=J_{0} J_{i} J_{j}$, which allows us to rewrite (6.10) as $\tilde{\alpha}_{j}^{0}=\sum_{i=1}^{r-2} d_{i} \tilde{\alpha}_{j}^{i}$ for $j=0, \ldots, r-2$.

6.2. The quantum $\mathbb{Z}$-variation of Hodge structure. - Following [CK, §8] and [Ir, §5], we now introduce a weight 3 variation of Hodge structure on $V_{\mathcal{O}}=$ $V \otimes \mathcal{O}\left(\left(\Delta^{*}\right)^{r-1}\right)$, where the $\Delta^{*}$ are punctured disks with coordinates $q_{j}=e^{2 \pi \mathbf{i} \tau_{j}}$, $j=0, \ldots, r-2$. (Write $\kappa: \mathfrak{H}^{r-1} \rightarrow\left(\Delta^{*}\right)^{r-1}$ for the obvious map sending $\underline{\tau} \mapsto \underline{q}$.) The Hodge filtration is straightforward, given by

$$
F^{p} V_{\mathcal{O}}:=\oplus_{a \geq p} H^{3-a, 3-a}\left(\mathrm{X}^{\circ}\right)_{\mathcal{O}}
$$


so that $1_{\mathrm{X}^{\circ}}=\left[\mathrm{X}^{\circ}\right](\otimes 1)$ generates $F^{3}$. The polarization is just $(\mathrm{A}, \mathrm{B}):=$ $(-1)^{\frac{1}{2} \operatorname{deg} \mathrm{A}} \int_{\mathrm{X}^{\circ}} \mathrm{A} \cdot \mathrm{B}$.

Let $\tilde{N}_{\hat{k}}$ denote the genus-zero Gromov-Witten invariant for the class $C_{\hat{k}}:=\sum_{\ell=0}^{r-2} k_{\ell} C_{\ell} \in$ $H_{2}\left(\mathrm{X}^{\circ}, \underline{\mathbb{Z}}\right)$, for any $\underline{\hat{k}}=\left(k_{0}, \underline{k}\right) \in \mathbb{Z}_{\geq 0}^{r-1}$. Using the Gromov-Witten prepotential

$$
\Phi:=\frac{(2 \pi \mathbf{i})^{3}}{6} \int_{\mathrm{X}^{\circ}}\left(\sum_{j=0}^{r-2} \tau_{j} J_{j}\right)^{3}+\sum_{\underline{k} \neq \underline{0}} \tilde{N}_{\underline{\hat{k}}} \underline{q}^{\underline{\hat{k}}},
$$

we define the quantum product "*” on $V_{\mathcal{O}}$ to be cup product on the last subsection's basis $(\otimes 1)$ except for

$$
J_{i} * J_{j}:=\frac{1}{(2 \pi \mathbf{i})^{3}} \sum_{\ell=0}^{r-2} \Phi_{i j \ell}^{\prime \prime \prime} C_{\ell}=J_{i} \cdot J_{j}+\text { h.o.t. }(\underline{q}) .
$$

where h.o.t. $(\underline{q})$ denote higher order term in the $q$ expansion. Here $\Phi_{i j \ell}^{\prime \prime \prime}=\partial_{i} \partial_{j} \partial_{\ell} \Phi$, where $\partial_{i}:=\frac{\bar{\partial}}{\partial q_{i}}$.

The ( $\mathbb{C}$-)local system $\mathbb{V}_{\mathbb{C}} \subset V_{\mathcal{O}}$ is then given by the kernel of

$$
\nabla:=\mathrm{id}_{V} \otimes d+\sum_{i=0}^{r-2}\left(J_{i} *\right) \otimes d \tau_{i}
$$

with monodromy logarithms

$$
N_{i}=\log \left(T_{i}\right)=-2 \pi \mathbf{i} \operatorname{Res}_{q_{i}=0}(\nabla)=-J_{i} \cdot()
$$

about the coordinate axes. A basis of $\nabla$-flat sections is given by the map

$$
\sigma: B \rightarrow \Gamma\left(\mathfrak{H}^{r-1}, \kappa^{*} \mathbb{V}_{\mathbb{C}}\right)
$$

sending

$$
\left\{\begin{array}{l}
p \mapsto p \\
C_{i} \mapsto C_{i}-\tau_{i} p \\
J_{i} \mapsto J_{i}-\frac{1}{(2 \pi \mathbf{i})^{3}} \sum_{j} \Phi_{i j}^{\prime \prime} C_{j}+\frac{1}{(2 \pi \mathbf{i})^{3}} \Phi_{i}^{\prime} p \\
\mathrm{X}^{\circ} \mapsto \mathrm{X}^{\circ}-\sum_{i} \tau_{i} J_{i}-\frac{1}{(2 \pi \mathbf{i})^{3}} \sum_{i}\left(\Phi_{i}^{\prime}-\sum_{j} \tau_{j} \Phi_{i j}^{\prime \prime}\right) C_{i} \\
\quad+\frac{1}{(2 \pi \mathbf{i})^{3}}\left(2 \Phi-\sum_{j} \tau_{j} \Phi_{j}^{\prime}\right) p
\end{array}\right.
$$

To define Iritani's integral local system $\mathbb{V}_{\mathbb{Z}} \subset \mathbb{V}_{\mathbb{C}}$, we will need his "square root of the Todd class"

$$
\hat{\Gamma}\left(\mathrm{X}^{\circ}\right):=\exp \left(-\frac{1}{24} c h_{2}\left(\mathrm{X}^{\circ}\right)-\frac{2 \zeta(3)}{(2 \pi \mathbf{i})^{3}} c h_{3}\left(\mathrm{X}^{\circ}\right)\right) \in K_{0}\left(\mathrm{X}^{\circ}\right) .
$$


In general, for a toric variety $\mathbb{P}_{\Sigma}$ defined by a simplicial fan $\Sigma$,

$$
c_{i}\left(\mathbb{P}_{\Sigma}\right)=\sum_{\tau \in \Sigma^{(i)}} Z_{\tau}
$$

where $\Sigma^{(i)}$ denotes the $i$-dimensional cones of $\Sigma$ and $Z_{\tau}$ is the (codimension- $i$ ) intersection of the divisors of $\mathbb{P}_{\Sigma}$ dual to the generators of $\tau$. Applying this to $\mathbb{P}_{\hat{\Delta}}$ and pulling back to $\mathrm{X}^{\circ}$,

$$
\begin{aligned}
\imath^{*} c\left(\mathbb{P}_{\hat{\Delta}}\right)= & +\left(D^{\prime}+D^{\prime \prime}+\sum_{i=0}^{r} D_{i}\right) \\
& +\left(\left(11 r^{\circ}+r\right) C_{0}+12 \sum_{i=1}^{r} C_{i}\right)+6\left(r+r^{\circ}\right) p
\end{aligned}
$$

while

$$
\imath^{*} c\left(\mathcal{O}\left(\mathrm{X}^{\circ}\right)^{-1}\right)=1-6 \sum_{i=0}^{r} D_{i}+36\left(r^{\circ} C_{0}+\sum_{i=1}^{r} C_{i}\right)-216 r^{\circ} p .
$$

(Remark that $\sum_{i=1}^{r} C_{i}=\sum_{i=1}^{r-2} \alpha_{0}^{i} C_{i}$ by (6.6).) This yields

$$
c\left(\mathrm{X}^{\circ}\right)=\imath^{*} c\left(\mathbb{P}_{\hat{\Delta}}\right) \cdot c\left(\mathcal{O}\left(\mathrm{X}^{\circ}\right)\right)^{-1}=1+\left(\left(11 r^{\circ}+r\right) C_{0}+12 \sum_{i=1}^{r} C_{i}\right)-60 r^{\circ} p,
$$

hence

$$
\left\{\begin{array}{l}
\operatorname{ch}\left(\mathrm{X}^{\circ}\right)=3-\left(12 \sum_{i=1}^{r} C_{i}+\left(11 r^{\circ}+r\right) C_{0}\right)-30 r^{\circ} p \\
t d\left(\mathrm{X}^{\circ}\right)=1+\left(\sum_{i=1}^{r} C_{i}+\frac{1}{12}\left(11 r^{\circ}+r\right) C_{0}\right) \\
\hat{\Gamma}\left(\mathrm{X}^{\circ}\right)=1+\left(\frac{1}{2} \sum_{i=1}^{r} C_{i}+\frac{1}{24}\left(11 r^{\circ}+r\right) C_{0}\right)+\frac{60 \zeta(3)}{(2 \pi \mathbf{i})^{3}} r^{\circ} p .
\end{array}\right.
$$

The $\mathbb{Z}$-local system (or rather its $\kappa^{*}$-pullback) is then defined by the image of

$$
\begin{array}{cl}
\gamma: K_{0}^{\text {num }}\left(\mathrm{X}^{\circ}\right) & \rightarrow \Gamma\left(\mathfrak{H}^{r-1}, \kappa^{*} \mathbb{V}_{\mathbb{C}}\right) \\
\xi & \mapsto \sigma\left(\hat{\Gamma}\left(\mathrm{X}^{\circ}\right) \cdot \operatorname{ch}(\xi)\right)
\end{array}
$$

The role of the $\hat{\Gamma}$-class is tied to the Mukai pairing $\langle\rangle:, K_{0}^{\text {num }}\left(\mathrm{X}^{\circ}\right) \times K_{0}^{\text {num }}\left(\mathrm{X}^{\circ}\right) \rightarrow \mathbb{Z}$, defined (on the level of vector bundles) by

$$
\left\langle\xi, \xi^{\prime}\right\rangle:=\int_{\mathrm{X}} \operatorname{ch}\left(\xi^{\vee} \otimes \xi^{\prime}\right) \cdot t d\left(\mathrm{X}^{\circ}\right)
$$

Iritani's result that

$$
\left(\gamma(\xi), \gamma\left(\xi^{\prime}\right)\right)=\left\langle\xi, \xi^{\prime}\right\rangle
$$

implies the integrality of $($,$) on \mathbb{V}_{\mathbb{Z}}$, and the integrality of monodromy follows from

$$
T_{i}(\gamma(\xi))=\gamma\left(\mathcal{O}\left(-J_{i}\right) \otimes \xi\right) .
$$

The "period" of the $(3,0)$-section $\left[\mathrm{X}^{\circ}\right] \otimes 1$ against the integral class $\gamma(\xi)$ is

$$
\Pi_{\xi}^{\mathrm{A}}(\underline{q}):=\left\langle 1_{\mathrm{X}^{\circ}}, \gamma(\xi)\right\rangle=\text { coefficient of }[p] \text { in } \gamma(\xi) .
$$


To compute $\gamma$ or the Mukai pairing, we first find the Chern characters of various skyscraper sheaves by resolving them by vector bundles, e.g.

$$
\mathcal{O}_{J_{i}}=\mathcal{O}-\mathcal{O}\left(-J_{i}\right), \quad \mathcal{O}_{C_{i}}=\mathcal{O}-\mathcal{O}\left(-D_{i}\right)-\mathcal{O}\left(-D_{0}\right)+\mathcal{O}\left(-\left(D_{0}+D_{i}\right)\right),
$$

and using $\operatorname{ch}(\mathcal{O}(D))=e^{D}$ for any divisor $D$. Writing $\ell_{j}^{i}:=\left(C_{i} \cdot D_{j}\right)(i, j=0, \ldots, r),{ }^{(11)}$ this gives

$$
\begin{aligned}
& \operatorname{ch}\left(\mathcal{O}_{p}\right)=p, \quad \operatorname{ch}\left(\mathcal{O}_{\mathrm{X}^{\circ}}\right)=\mathrm{X}^{\circ}, \\
& \operatorname{ch}\left(\mathcal{O}_{C_{0}}\right)=C_{0}, \quad \operatorname{ch}\left(\mathcal{O}_{C_{j}}\right)=C_{j}+\frac{1}{2}\left(d_{j}-\ell_{j}^{j}\right) p, \\
& \operatorname{ch}\left(\mathcal{O}_{J_{0}}\right)=J_{0}-\frac{1}{2}\left(r^{\circ} C_{0}+\Sigma_{i=1}^{r} C_{i}\right)+\frac{r^{\circ}}{6} p, \quad \operatorname{ch}\left(\mathcal{O}_{J_{j}}\right)=J_{j}-\frac{1}{2} \alpha_{j}^{j} C_{0}, \\
& \operatorname{ch}\left(\mathcal{O}_{D_{0}}\right)=D_{0}+\frac{1}{2} \Sigma_{i=1}^{r} C_{i}+p, \quad \operatorname{ch}\left(\mathcal{O}_{D_{j}}\right)=D_{j}+\frac{1}{2} C_{0} .
\end{aligned}
$$

In particular, we find that a Mukai-symplecitic basis $\left\{\xi_{k}\right\}_{k=1}^{2 r}$ of $K_{0}^{\text {num }}\left(\mathrm{X}^{\circ}\right)_{\mathbb{Q}}$ (hence, applying $\gamma$, a symplectic basis of $\left.\mathbb{V}_{\mathbb{Q}}\right)$, with ${ }^{(12)}$

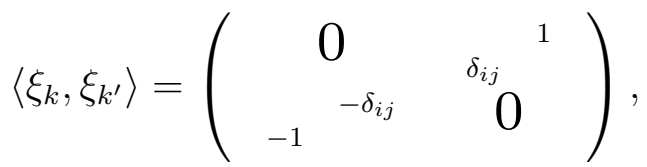

is given by

$$
\left\{\begin{array}{l}
\xi_{1}=\mathcal{O}_{\mathrm{X}^{\circ}} \\
\quad \xi_{2}=\mathcal{O}_{J_{0}}+\frac{1}{4} \sum_{j=1}^{r-2}\left(\alpha_{0}^{j}-\alpha_{j}^{j}\right) \mathcal{O}_{C_{j}} \\
\quad \quad-\left(\frac{13 r^{\circ}+r}{12}+\frac{1}{2} \sum_{j=1}^{r-2}\left(\alpha_{0}^{j}-\alpha_{j}^{j}\right)\left(d_{j}-\ell_{j}^{j}\right)\right) \mathcal{O}_{p} \\
\quad \xi_{2+j}=\mathcal{O}_{J_{j}}+\frac{1}{4}\left(\alpha_{j}^{j}-\alpha_{0}^{j}\right) \mathcal{O}_{C_{0}}-\alpha_{0}^{j} \mathcal{O}_{p} \quad(j=1, \ldots, r-2) \\
\xi_{r+1}=-\mathcal{O}_{C_{0}} \\
\xi_{r+j+1}=-\mathcal{O}_{C_{j}}+\frac{1}{2}\left(d_{j}-\ell_{j}^{j}\right) \mathcal{O}_{p} \quad(j=1, \ldots, r-2) \\
\xi_{2 r}=\mathcal{O}_{p} .
\end{array}\right.
$$

However, the basis given by the skyscraper sheaves themselves is adequate for purposes of analyzing monodromy. We shall also have use for the partial basis

$$
\left\{\begin{array}{l}
\hat{\xi}_{D_{0}}=\mathcal{O}_{D_{0}}+\frac{1}{2} \sum_{j=1}^{r-2} \alpha_{0}^{j} C_{j}+\left(-\frac{15 r^{\circ}+r}{24}+\frac{1}{4} \sum_{j=1}^{r-2} \alpha_{0}^{j}\left(\ell_{j}^{j}+d_{j}\right)\right) \mathcal{O}_{p} \\
-\hat{\xi}_{C_{j}}=\xi_{r+j+1} \quad(j=0, \ldots, r=2) \\
\hat{\xi}_{p}=\mathcal{O}_{p}
\end{array}\right.
$$

later on. It satisfies

$$
\operatorname{ch}\left(\hat{\xi}_{D_{0}}\right) \cdot \hat{\Gamma}=D_{0}, \quad \operatorname{ch}\left(\hat{\xi}_{C_{j}}\right) \cdot \hat{\Gamma}=-C_{j}, \quad \operatorname{ch}\left(\hat{\xi}_{p}\right) \cdot \hat{\Gamma}=p,
$$

\footnotetext{
$\overline{(11)}$ note $\ell_{0}^{i}=d_{i}$

${ }^{(12)}$ in the matrix, $i$ and $j$ run from 0 to $r-2$
} 
which implies

$$
\gamma\left(\hat{\xi}_{D_{0}}\right)=\sigma\left(D_{0}\right), \quad \gamma\left(\hat{\xi}_{C_{j}}\right)=-\sigma\left(C_{j}\right), \quad \gamma\left(\hat{\xi}_{p}\right)=\sigma(p) .
$$

In particular, we have

$$
\Pi_{\hat{\xi}_{C_{j}}}^{\mathrm{A}}=\tau_{j} \quad \text { and } \quad \Pi_{\hat{\xi}_{p}}^{\mathrm{A}} \equiv 1
$$

In the sequel the $\mathbb{Z}$-variation of Hodge structure $\left(\mathbb{V}_{\mathbb{Z}}, V_{\mathcal{O}}, F^{\bullet}\right)$ constructed above will be denoted $\mathcal{V}_{\mathrm{A}}$.

6.3. Monodromy types. - We shall compute monodromy directly on the level of $K_{0}^{\text {num }}\left(\mathrm{X}^{\circ}\right)_{\mathbb{Q}}$, by applying $\mathcal{O}\left(-J_{j}\right) \otimes$ to the basis

$$
\mathcal{O}_{\mathrm{X} \circ} ; \mathcal{O}_{J_{0}}, \ldots, \mathcal{O}_{J_{r-2}} ; \mathcal{O}_{C_{0}}, \ldots, \mathcal{O}_{C_{r-2}} ; \mathcal{O}_{p}
$$

Writing $i$ resp. $k$ for the rows resp. columns of the various blocks, this gives

$$
T_{j}=\left(\begin{array}{cccc}
1 & 0 & 0 & 0 \\
-\delta_{i}^{j} & \delta_{i}^{k} & 0 & 0 \\
0 & -J_{i} J_{j} J_{k} & \delta_{i}^{k} & 0 \\
0 & 0 & -\delta_{j}^{k} & 1
\end{array}\right),
$$

where we note that $J_{i} J_{j} J_{k}$ is

$$
\tilde{\alpha}_{i}^{k}=\left(\begin{array}{cc}
r^{\circ} & \alpha_{0}^{k} \\
\alpha_{0}^{i} & \alpha_{i}^{k}
\end{array}\right) \quad \operatorname{resp} \cdot\left(\begin{array}{cc}
\alpha_{0}^{j} & \alpha_{k}^{j} \\
\alpha_{i}^{j} & 0
\end{array}\right)
$$

if $j=0$ resp. $1, \ldots, r-2$. So

$$
\begin{aligned}
N_{j} & =\left(T_{j}-I\right)-\frac{1}{2}\left(T_{j}-I\right)^{2}+\frac{1}{3}\left(T_{j}-I\right)^{3} \\
& =\left(\begin{array}{cccc}
0 & 0 & 0 & 0 \\
-\delta_{i}^{j} & 0 & 0 & 0 \\
-\frac{1}{2} J_{j}^{2} J_{i} & -J_{i} J_{j} J_{k} & 0 & 0 \\
-\frac{1}{3} J_{j}^{3} & -\frac{1}{2} J_{j}^{2} J_{k} & -\delta_{j}^{k} & 0
\end{array}\right)
\end{aligned}
$$

and the ensuing monodromy weight filtrations $W\left(N_{j}\right)$ • are rather different in these two cases, ${ }^{(13)}$ which we denote type "I" resp. "II".

$\overline{{ }^{(13)} \text { We remind }}$ the reader that if $j>0, J_{j}^{2} J_{i}=\alpha_{j}^{j} \delta_{i}^{0}$ and $J_{j}^{3}=0$, while if $j=0$ then $J_{0}^{2} J_{i}=\alpha_{0}^{i}$ and $J_{0}^{3}=r^{\circ}$. 
For $W_{\bullet}=W\left(N_{0}\right) \bullet$ (type I), we determine the following generators for the $G r_{\ell}^{W}$ :

$$
\begin{aligned}
& W_{0}=\left\langle\mathcal{O}_{p}\right\rangle \\
& W_{2}=W_{0}+\left\langle\left\{\Sigma_{k=1}^{r-2}\left(\alpha_{0}^{i} \alpha_{i+1}^{k}-\alpha_{0}^{i+1} \alpha_{i}^{k}\right) \mathcal{O}_{C_{k}}\right\}_{i=1}^{r-3}, r^{\circ} \mathcal{O}_{C_{0}}+\Sigma_{k=1}^{r-2} \alpha_{0}^{k} \mathcal{O}_{C_{k}}\right\rangle \\
& W_{3}=W_{2}+\left\langle\mathcal{O}_{D_{0}}, \Sigma_{k, i=1}^{r-2} d_{i} \alpha_{k}^{i} \mathcal{O}_{C_{k}}\right\rangle \\
& W_{4}=W_{3}+\left\langle\left\{\alpha_{0}^{i+1} \mathcal{O}_{J_{i}}-\alpha_{0}^{i} \mathcal{O}_{J_{i+1}}\right\}_{i=1}^{r-3}, \mathcal{O}_{J_{0}}\right\rangle \\
& W_{6}=W_{4}+\left\langle\mathcal{O}_{\mathrm{X}}\right\rangle .
\end{aligned}
$$

The $T_{0}$-invariants $\operatorname{ker}\left(T_{0}-I\right)=\operatorname{ker}\left(N_{0}\right)$ are spanned by

$$
\mathcal{O}_{p},\left\{\mathcal{O}_{C_{k}}\right\}_{k=1}^{r-2} \text {, and } \mathcal{O}_{D_{0}}
$$

A key point here is that because the " $\zeta(3)$ " in $\hat{\Gamma}\left(\mathrm{X}^{\circ}\right)$ only appears in $\gamma\left(\mathcal{O}_{\mathrm{X}^{\circ}}\right)$, it does not appear in any $T_{0}$-invariant A-model periods.

For $W_{\bullet}=W\left(N_{j}\right) \bullet(j>0)$, the situation bifurcates according to whether $\alpha_{j}^{j} \neq 0$ (type IIa) or $\alpha_{j}^{j}=0$ (type IIb). If $\alpha_{j}^{j} \neq 0$ then we have $W_{0}=\{0\}$,

$$
\begin{aligned}
& W_{1}=\left\langle\mathcal{O}_{p}, \mathcal{O}_{C_{0}}\right\rangle, \\
& W_{3}=W_{1}+\left\langle\Sigma_{k=0}^{r-2} \alpha_{k}^{j} \mathcal{O}_{C_{k}}, \mathcal{O}_{J_{j}},\left\{\mathcal{O}_{C_{i}}\right\}_{i \neq j, 0},\left\{\alpha_{i+1}^{j} \mathcal{O}_{J_{i}}-\alpha_{i}^{j} \mathcal{O}_{J_{i+1}}\right\}_{i=1}^{r-3}\right\rangle, \\
& W_{5}=W_{3}+\left\langle\mathcal{O}_{J_{0}}, \mathcal{O}_{\mathrm{X}}\right\rangle .
\end{aligned}
$$

In particular, $N_{j}$ sends $\mathcal{O}_{J_{0}} \mapsto-\sum \alpha_{k}^{j} \mathcal{O}_{C_{k}} \mapsto \alpha_{j}^{j} \mathcal{O}_{p}$ and $\mathcal{O}_{\mathrm{X}} \mapsto-\mathcal{O}_{J_{j}} \mapsto \alpha_{j}^{j} \mathcal{O}_{C_{0}}$. A basis for the $T_{j}$-invariants in this case (type IIa) is ${ }^{(14)}$

$$
\mathcal{O}_{p},\left\{\mathcal{O}_{C_{i}}\right\}_{i \neq j} \text {, and }\left\{\alpha_{i+1}^{j} \mathcal{O}_{J_{i}}-\alpha_{i}^{j} \mathcal{O}_{J_{i+1}}\right\}_{i=1}^{r-3} \text {. }
$$

If $\alpha_{j}^{j}=0$, let $j^{\prime} \neq j, 0$ be such that $\alpha_{j^{\prime}}^{j} \neq 0$; this exists because $\left\{\alpha_{\ell}^{k}\right\}_{k, \ell=1}^{r-2}$ is nondegenerate. The type IIb weight filtration is then $W_{1}=\{0\}$,

$$
\begin{aligned}
& W_{2}=\left\langle\mathcal{O}_{p}, \mathcal{O}_{C_{0}}, \Sigma_{k=1}^{r-2} \alpha_{k}^{j} \mathcal{O}_{C_{k}}, \mathcal{O}_{J_{j}}\right\rangle \\
& W_{3}=W_{2}+\left\langle\left\{\mathcal{O}_{C_{i}}\right\}_{i \neq 0, j, j^{\prime}},\left\{\alpha_{i}^{j} \mathcal{O}_{J_{j^{\prime}}}-\alpha_{j^{\prime}}^{j} \mathcal{O}_{J_{i}}\right\}_{i \neq 0, j, j^{\prime}}\right\rangle \\
& W_{4}=W_{3}+\left\langle\mathcal{O}_{\mathrm{X}}, \mathcal{O}_{J_{0}}, \mathcal{O}_{J_{j^{\prime}}}, \mathcal{O}_{C_{j}}\right\rangle
\end{aligned}
$$

with $T_{j}$-invariants

$$
\mathcal{O}_{p},\left\{\mathcal{O}_{C_{i}}\right\}_{i \neq j}, \mathcal{O}_{J_{j}},\left\{\alpha_{i}^{j} \mathcal{O}_{J_{j^{\prime}}}-\alpha_{j^{\prime}}^{j} \mathcal{O}_{J_{i}}\right\}_{i \neq 0, j, j^{\prime}}
$$

The three types of limiting mixed Hodge structure $\psi_{q_{j}} \mathcal{V}_{\mathrm{A}}$ (arising along the hyperplanes $\left.\left\{q_{j}=0\right\}\right)$ can be displayed pictorially by placing a bullet in the $(p, q)$ spot if

$\overline{(14)\left\{\mathcal{O}_{C_{i}}\right\}_{i \neq j}}$ includes $\mathcal{O}_{C_{0}}$ 
$\left(\psi_{q_{j}} \mathcal{V}_{\mathrm{A}}\right)^{p, q} \neq\{0\}$ (and indicating its rank). Arrows denote the action of $N_{j}$, with ranks of these maps indicated:

(I)

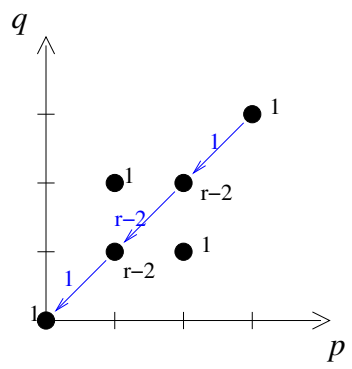

(IIa)

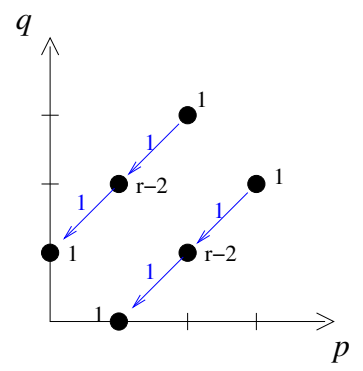

(IIb)

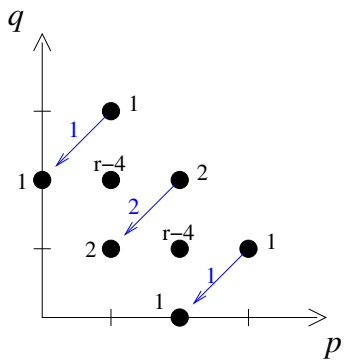

Note that the space ofinvariants (i.e. $\operatorname{ker}(N)$ ) has rank $r$ for type I but rank $2 r-4$ for both types IIa and IIb. Bases forthese invariant spaces may obviously be obtained by applying $\gamma$ to (6.25)-(6.27), and changing basis where convenient. For type I, we find immediately that $(6.23)$ is a basis for $\left(\kappa^{*}\right.$ of $) \operatorname{ker}\left(N_{0}\right) \subset \mathbb{V}_{\mathbb{Q}}$. For both types II, one deduces that

$$
\sigma(p),\left\{\sigma\left(C_{i}\right)\right\}_{i \neq j}\left(\text { incl. } \sigma\left(C_{0}\right)\right) \text {, and }
$$

$$
r-3 \mathbb{Q} \text {-linear combinations of the }\left\{\sigma\left(J_{i}\right)\right\}_{i=1}^{r-2}
$$

$\operatorname{span} \operatorname{ker}\left(N_{j}\right)$.

6.4. Mirror map. — Let $\square \subset \mathbb{R}^{n}$ be a reflexive polytope, $\mathfrak{F}=\sum_{i=0}^{m+n} b_{i} \underline{\underline{\mathrm{x}}}^{(i)}$ a general $\square$-regular Laurent polynomial (with $\underline{\mathrm{v}}^{(0)}=\underline{0}$ ), and assume none of the $\left\{\underline{\mathrm{v}}^{(i)}\right\}_{i=0}^{m+n}=$ $\square \cap \mathbb{Z}^{n}$ lie on the relative interior of a facet of $\square$. If $\mathrm{V}$ denotes the $\mathbb{Q}$-vector space formally generated by the $\left\{\underline{\mathrm{v}}^{(i)}\right\}_{i=1}^{m+n}$, let $\mathrm{R}:=\operatorname{ker}\left\{\mathrm{V} \rightarrow \mathbb{Q}^{n}\right\}$ be the relations subspace, with $\mathbb{Q}$-basis $\left\{\underline{\underline{r}}^{(j)}=\sum_{i=1}^{m+n} \mathbf{r}_{i}^{(j)} \underline{\mathbf{v}}^{(i)}\right\}_{j=1}^{m}$, and set $($ for $j=1, \ldots, m)$

$$
w_{j}:=b_{0}^{-\sum_{i} r_{i}^{(j)}} \prod_{i} b_{i}^{\mathbf{r}_{i}^{(j)}}=\prod_{i}\left(\frac{b_{i}}{b_{0}}\right)^{\mathbf{r}_{i}^{(j)}} .
$$

Write $\mathcal{X} \subset \mathbb{P}_{\square}$ for the zero locus of $\mathfrak{F}$ and $\mathcal{X}^{\circ} \subset \mathbb{P}_{\square \circ \text { for a general anticanonical }}$ hypersurface. We have the exact sequence

$$
0 \rightarrow\left(\mathbb{Q}^{n}\right)^{\vee} \rightarrow \mathrm{V}^{\vee} \rightarrow \mathrm{R}^{\vee} \rightarrow 0
$$

where $\mathrm{R}^{\vee} \cong H^{2}\left(\mathcal{X}^{\circ}, \mathbb{Q}\right)$. A basis of $\mathrm{V}^{\vee}$ is given by the divisors $\mathcal{D}_{i} \subset \mathcal{X}^{\circ}$ dual to the $\underline{\mathrm{v}}^{(i)}$. Choose $\left\{\beta_{\ell}^{k}\right\} \in \mathbb{Q}^{m(m+n)}$ such that $\sum_{k=1}^{m+n} \beta_{\ell}^{k} r_{k}^{(j)}=\delta_{\ell}^{j}(\ell, j=1, \ldots, m)$, and put

$$
\mathcal{J}_{\ell}:=\sum_{k=1}^{m+n} \beta_{\ell}^{k}\left[\mathcal{D}_{k}\right] \in H^{2}\left(\mathcal{X}^{\circ}\right) \text {. }
$$


This gives a basis dual to $\left\{\underline{\underline{r}}^{(j)}\right\}$, since

$$
\underline{\underline{\mathbf{r}}}^{(j)}\left(\mathcal{J}_{\ell}\right)=\sum_{i, k} \beta_{\ell}^{k} \mathrm{r}_{i}^{(j)} \underline{\mathrm{v}}^{(i)}\left(\mathcal{D}_{k}\right)=\sum_{i, k} \beta_{\ell}^{k} \mathrm{r}_{i}^{(j)} \delta_{k}^{i}=\sum_{k} \beta_{\ell}^{k} \mathrm{r}_{k}^{(j)}=\delta_{\ell}^{j}
$$

Now the mirror map sends the complex structure parameter $\underline{b}$ of $\mathcal{X}$ to a Kähler parameter in $H^{2}\left(\mathcal{X}^{\circ}, \mathbb{C}\right)$, of the form $\tau(\underline{w})=$

$$
=\sum_{j=1}^{m} \tau_{j}(\underline{w}) \mathcal{J}_{j}=\frac{1}{2 \pi \mathbf{i}} \sum_{i=1}^{m+n} \log \left(\frac{b_{i}}{b_{0}}\right)\left[\mathcal{D}_{i}\right]+\mathcal{O}\left(\left\{\frac{b_{i}}{b_{0}}\right\}\right),
$$

where $\tau_{j}(\underline{b})$ are (B-model) periods. We compute

$$
\begin{aligned}
\underline{\underline{\mathbf{r}}}^{(j)}\left(\sum_{i} \log \left(\frac{b_{i}}{b_{0}}\right)\left[\mathcal{D}_{i}\right]\right) & =\sum_{i, k} \mathbf{r}_{k}^{(j)} \log \left(\frac{b_{i}}{b_{0}}\right) \underline{\mathbf{v}}^{(k)}\left(\mathcal{D}_{i}\right) \\
& =\sum_{i} \boldsymbol{r}_{i}^{(j)} \log \left(\frac{b_{i}}{b_{0}}\right)=\log \left(w_{j}\right),
\end{aligned}
$$

which shows $\tau_{j}(\underline{w}) \sim \frac{1}{2 \pi \mathbf{i}} \log \left(w_{j}\right)$.

Applying this to our situation $(n=4, m=r-1)$, with $\alpha, \beta, \gamma,\left\{a_{i}\right\}$ replacing the $\left\{b_{i}\right\}$, with $D^{\prime}, D^{\prime \prime}, D_{0}, \ldots, D_{r}$ replacing the $\left\{\mathcal{D}_{i}\right\}$, and with $z_{0}, \ldots, z_{r-2}$ replacing $w_{1}, \ldots, w_{m}$, we recover (5.2) and (6.4), and find that the coefficients $\left\{\tau_{j}(\underline{z})\right\}$ of the $\left\{J_{j}\right\}$ in $\tau(\underline{z})$ are asymptotic to $\frac{1}{2 \pi \mathbf{i}} \log \left(z_{j}\right)$. By $\S 5.7$ (especially (5.16)-(5.17)), the mirror map is therefore exactly

$$
\tau(\underline{z})=\sum_{j=0}^{r-2} \tau_{j}(\underline{z}) J_{j}=\Pi_{\tau_{\beta}}^{\mathrm{B}}(\underline{z}) J_{0}+\sum_{j=1}^{r-2} \Pi_{\mathcal{M}_{\alpha}\left(\varphi_{0}^{(j)}\right)}^{\mathrm{B}}(\underline{z}) J_{j} .
$$

Writing $\mathcal{Q}\left(z_{0}, \ldots, z_{r-2}\right):=\left(q_{0}(\underline{z}), \ldots, q_{r-2}(\underline{z})\right)$, we note that the B-model coordinate axes $z_{j}=0$ map to the A-model axes $q_{j}=0$.

Now [Ir, Thm. 5.9] provides an isomorphism $\Theta: \mathcal{Q}^{*} \mathcal{V}_{\mathrm{A}} \stackrel{\cong}{\rightarrow} \mathcal{V}_{\mathrm{B}}$ of $\mathbb{Z}-$ variation of Hodge structure sending $1_{\mathrm{X}^{\circ}} \mapsto[\tilde{\Omega}]$. Since (6.24) and (6.28) identify the periods

$$
\Pi_{\mathcal{T}_{\beta}}^{\mathrm{B}}(\underline{z}) \equiv \Pi_{\hat{\xi}_{C_{0}}}^{\mathrm{A}}(\mathcal{Q}(\underline{z})) \quad \text { and } \quad \Pi_{\mathcal{M}_{\alpha}\left(\varphi_{0}^{(j)}\right)}^{\mathrm{B}}(\underline{z}) \equiv \Pi_{\hat{\xi}_{C_{j}}}^{\mathrm{A}}(\mathcal{Q}(\underline{z}))
$$

modulo $\mathbb{Z}$, and obviously $\Pi_{\mathcal{T}_{\alpha}}^{\mathrm{B}}(\underline{z})=1=\Pi_{\hat{\xi}_{p}}^{\mathrm{A}}\left(\mathcal{Q}(\underline{z})\right.$ ), we deduce that (up to changing $\mathcal{T}_{\beta}$ and $\mathcal{M}_{\alpha}\left(\varphi_{0}^{(j)}\right)$ by integer multiples of $\left.\mathcal{T}_{\alpha}\right)$

$$
\Theta(\sigma(p))=\mathcal{T}_{\alpha}, \quad \Theta\left(-\sigma\left(C_{0}\right)\right)=\mathcal{T}_{\beta}, \quad \Theta\left(-\sigma\left(C_{j}\right)\right)=\mathcal{M}_{\alpha}\left(\varphi_{0}^{(j)}\right)
$$

By considering $W\left(N_{0}\right)$ • on $\operatorname{ker}\left(N_{0}\right)$ on the $\mathrm{A}$ and B sides (cf. $\S 5.6$ and $\S 6.3$ ), we find in addition that (after modifying $\varphi_{1}$ by $\mathbb{Z}\left\langle\left\{\varphi_{0}^{(i)}\right\}\right\rangle$ and $\mathcal{M}_{\alpha}\left(\varphi_{1}\right)$ by $\mathbb{Z}\left\langle\mathcal{T}_{\alpha}\right\rangle$ if necessary)

$$
\Theta\left(\sigma\left(D_{0}\right)\right)=\Theta\left(\gamma\left(\hat{\xi}_{D_{0}}\right)\right)=\mathcal{M}_{\alpha}\left(\varphi_{1}\right)
$$


(More precisely, if we look at $W\left(N_{0}\right)_{3} \cap \operatorname{ker}\left(T_{0}-I\right)$ in $H^{3}(\mathrm{X}, \mathbb{Z})\left(\subset \mathcal{V}_{\mathrm{B}}\right)$ resp. $\mathbb{V}_{\mathbb{Z}}\left(\subset \mathcal{V}_{\mathrm{A}}\right)$, this is generated by $\mathcal{M}_{\alpha}\left(\varphi_{1}\right) \bmod \mathbb{Z}\left\langle\mathcal{T}_{\alpha},\left\{\mathcal{M}_{\alpha}\left(\varphi_{0}^{(i)}\right)\right\}\right\rangle \operatorname{resp.} \gamma\left(\hat{\xi}_{D_{0}}\right) \bmod \mathbb{Z}\left\langle\gamma\left(\hat{\xi}_{p}\right),\left\{\gamma\left(\hat{\xi}_{C_{i}}\right)\right\}_{i=1}^{r-2}\right\rangle$. $)$ Heuristically, we obviously have some matching as well between the $\left\{\mathcal{M}_{\beta}\left(\varphi_{0}^{(i)}\right)\right\}$ and $\left\{\gamma\left(\mathcal{O}_{J_{j}}\right)\right\}$, and between $\mathcal{M}_{\beta}\left(\varphi_{1}\right)$ and $\gamma\left(\mathcal{O}_{\mathrm{X}^{\circ}}\right)$; but we will not dissect this further, as (6.29) shall now yield the local mirror symmetry identity we seek.

Recalling that $\hat{k}=\left(k_{0}, \underline{k}\right)$, write $\tilde{N}_{\underline{k}}$ for $\tilde{N}_{\hat{k}}$ when $k_{0}=0$; and referring to $\S 5.7$, write $R_{1}:=R_{\varphi_{1}}(\underline{z})$ resp. $R_{0}^{(i)}:=R_{\varphi_{0}^{(i)}}(\underline{z})$, where $\underline{z}=\left(z_{1}, \ldots, z_{r-2}\right)$ omits $z_{0}$. Accordingly, we shall change notation for $\left(z_{0}, \ldots, z_{r-2}\right)$ to $\underline{\hat{z}}=\left(z_{0}, \underline{z}\right)$. Define local Kähler parameters $Q_{i}:=e^{R_{0}^{(i)}}($ for $i=1, \ldots, r-2)$.

Theorem 6.1. - On the universal cover of $\left(\Delta^{*}\right)^{r-2}$ we have

$$
(2 \pi \mathbf{i}) R_{1}=\frac{1}{2} \sum_{i, j=1}^{r-2} \alpha_{j}^{i} R_{0}^{(i)} R_{0}^{(j)}-\sum_{\underline{k} \neq \underline{0}}\left(\sum_{i=1}^{r-2} d_{i} k_{i}\right) \tilde{N}_{\underline{k}} \underline{Q}^{\underline{k}} .
$$

Remark 6.2. - This is Conjecture 5.1 in [DK]; also see [CKYZ, Hos].

Proof. - Taking periods of (6.29) on both sides (with respect to $\tilde{\Omega}$ resp. $1_{\mathrm{X}}$ ) yields

$$
\Pi_{\mathcal{M}_{\alpha}\left(\varphi_{1}\right)}^{\mathrm{B}}(\underline{\hat{z}})=\Pi_{\hat{\xi}_{D_{0}}}^{\mathrm{A}}(\mathcal{Q}(\underline{\hat{z}}))=\left\langle 1_{\mathrm{X}^{\circ}}, \sigma\left(D_{0}\right)\right\rangle(\mathcal{Q}(\underline{\hat{z}})) .
$$

By (6.5),

which by (6.13)

$$
\sigma\left(D_{0}\right)=\sigma\left(J_{0}\right)-\sum_{i=1}^{r-2} d_{i} \sigma\left(J_{i}\right)
$$

$$
=D_{0}-\frac{1}{(2 \pi \mathbf{i})^{3}} \sum_{j}\left(\Phi_{0 j}^{\prime \prime}-\Sigma_{i} d_{i} \Phi_{i j}^{\prime \prime}\right) C_{j}+\frac{1}{(2 \pi \mathbf{i})^{3}}\left(\Phi_{0}^{\prime}-\Sigma_{i} d_{i} \Phi_{i}^{\prime}\right) p .
$$

Writing $\partial_{D_{0}}:=\partial_{0}-\sum d_{i} \partial_{i}$, the A-model period is then

$$
\left\langle 1_{\mathrm{X}^{\circ}}, \sigma\left(D_{0}\right)\right\rangle=\frac{1}{(2 \pi \mathbf{i})^{3}} \partial_{D_{0}} \Phi=\partial_{D_{0}}\left(\sum_{j=0}^{r-2} \tau_{j} J_{j}\right)^{3}+\frac{1}{(2 \pi \mathbf{i})^{3}} \partial_{D_{0}} \sum_{\underline{\hat{k}} \neq 0} \tilde{N}_{\underline{\hat{k}}} \underline{Q}^{\tilde{Q}^{\underline{k}}} .
$$

For the first term, $\partial_{D_{0}}$ of

$$
\frac{r^{\circ}}{6} \tau_{0}^{3}+\frac{1}{2} \tau_{0}^{2} \sum_{j=1}^{r-2} \alpha_{0}^{j} \tau_{j}+\frac{1}{2} \tau_{0} \sum_{i, j=1}^{r-2} \alpha_{j}^{i} \tau_{i} \tau_{j}
$$

is

$$
\frac{1}{2}\left(r^{\circ}-\Sigma_{i} d_{i} \alpha_{0}^{i}\right) \tau_{0}^{2}+\tau_{0} \sum_{j=1}^{r-2}\left(\alpha_{0}^{j}-\Sigma_{i} d_{i} \alpha_{j}^{i}\right) \tau_{j}+\frac{1}{2} \sum_{i, j=1}^{r-2} \alpha_{j}^{i} \tau_{i} \tau_{j}=\frac{1}{2} \sum_{i, j} \alpha_{j}^{i} \tau_{i} \tau_{j}
$$


by $(6.10)$; for the second we have $\frac{1}{(2 \pi \mathbf{i})^{2}} \sum_{\underline{\hat{k}} \neq 0}\left(k_{0}-\sum d_{i} k_{i}\right) \tilde{N}_{\underline{\hat{k}}} \underline{q}^{\underline{\hat{k}}}$. Pulling back by $\mathcal{Q}$ therefore gives (as multivalued functions of $\underline{\hat{z}}$ )

$$
\Pi_{\mathcal{M}_{\alpha}\left(\varphi_{1}\right)}^{\mathrm{B}}=\frac{1}{2} \sum_{i, j} \alpha_{j}^{i} \Pi_{\mathcal{M}_{\alpha}\left(\varphi_{0}^{(i)}\right)}^{\mathrm{B}} \Pi_{\mathcal{M}_{\alpha}\left(\varphi_{0}^{(j)}\right)}^{\mathrm{B}}+\frac{1}{(2 \pi \mathbf{i})^{2}} \sum_{\underline{\hat{k} \neq \underline{0}}}\left(k_{0}-\Sigma d_{i} k_{i}\right) \tilde{N}_{\underline{\hat{k}}} \underline{q}(\underline{\hat{z}})^{\hat{k}^{\hat{k}}},
$$

where $q_{j}(\underline{\hat{z}})=e^{2 \pi \mathbf{i} \prod_{\mathcal{M}_{\alpha}\left(\varphi_{0}^{(i)}\right)}^{\mathrm{B}}}$ and $q_{0}(\underline{\hat{z}})=e^{2 \pi \mathbf{i} \prod_{\mathcal{T}_{\beta}}^{\mathrm{B}}} \sim z_{0}$.

Now we pass to the limit $z_{0} \rightarrow 0$, where (6.31) essentially becomes an equality of extension classes of A- and B-model limiting mixed Hodge structure. (In particular, the limit on both sides is finite since these are periods of $T_{0}$-invariant cycles; this is also clear from the absence of $\tau_{0}=\Pi_{\mathcal{T}_{\beta}}^{\mathrm{B}}$ in any term.) Since $\lim _{z_{0} \rightarrow 0} q_{0}(\underline{\hat{z}})=0$, the $\sum_{\underline{\hat{k}}}$ becomes a $\sum_{\underline{k}}$, while by $\S 5.7$

$$
\lim _{z_{0} \rightarrow 0} \Pi_{\mathcal{M}_{\alpha}\left(\varphi_{1}\right)}^{\mathrm{B}}\left(z_{0}, \underline{z}\right)=\frac{1}{2 \pi \mathbf{i}} R_{1}(\underline{z}), \quad \lim _{z_{0} \rightarrow 0} \Pi_{\mathcal{M}_{\alpha}\left(\varphi_{0}^{(i)}\right)}^{\mathrm{B}}\left(z_{0}, \underline{z}\right)=\frac{1}{2 \pi \mathbf{i}} R_{0}^{(i)}(\underline{z})
$$

hence $\lim _{z_{0} \rightarrow 0} q_{i}\left(z_{0}, \underline{z}\right)=Q_{i}(\underline{z})$. So $\left.(2 \pi \mathbf{i})^{2} \cdot(6.31)\right|_{z_{0}=0}$ indeed yields our main result (6.30).

The Gromov-Witten invariants $\tilde{N}_{\underline{k}}$ "counting"(15) genus-0 curves of class $\sum_{i=1}^{r-2} k_{i}\left[C_{i}\right]$ on $\mathrm{X}^{\circ}$, may also be interpreted as local Gromov-Witten invariants of $D_{0} \cong \mathbb{P}_{\Delta^{\circ}}$, or equivalently as (usual) Gromov-Witten invariants of the 3 -fold $\mathbb{P}(\mathcal{O} \oplus$ $K_{\mathbb{P}_{\Delta^{\circ}}}$ ). With this interpretation, the right-hand-side of (6.30) (perhaps replacing $R_{0}^{(i)}$ by $\left.(2 \pi \mathbf{i}) \tau_{i}\right)$ is the local Gromov-Witten prepotential $\Phi_{l o c}$ of $\mathbb{P}_{\Delta^{\circ}}$.

6.5. The sunset case. — Specializing to the diagram

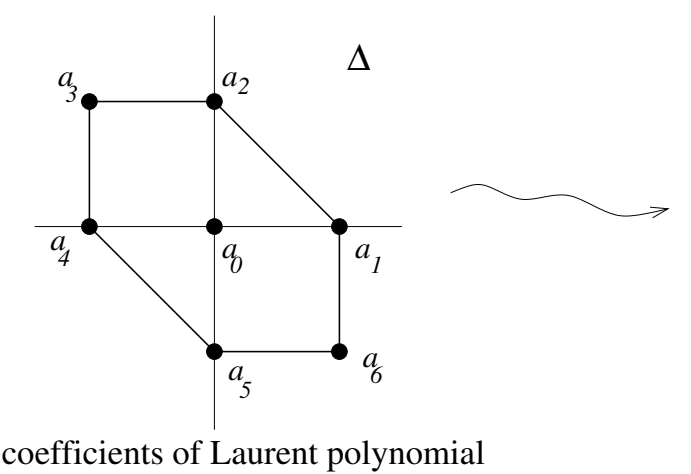

coefficients of Laurent polynomial

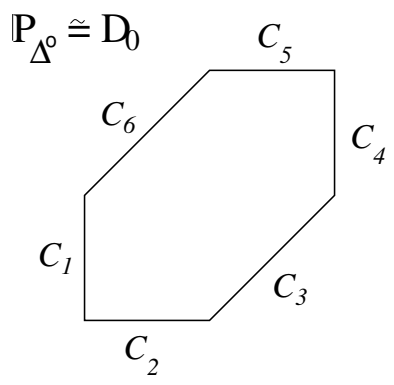

curves on the Fano surface

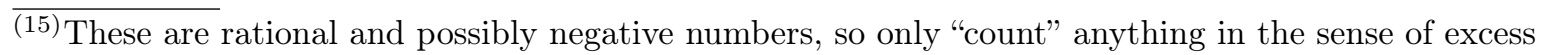
intersection number. 
we have $r=r^{\circ}=\nu=6, d_{i}=1$, and

$$
\ell_{j}^{i}= \begin{cases}-1, & i=j \\ 1, & i \underset{\overline{(6)}}{\equiv} \pm 1 \\ 0, & \text { otherwise. }\end{cases}
$$

From this we deduce that

$$
\begin{aligned}
& J_{1}=-D_{1}+D_{3}+D_{4}\left(=D_{6}\right), \quad J_{2}=D_{3}+D_{4}, \\
& J_{3}=D_{1}+D_{2}, \quad J_{4}=D_{1}+D_{2}-D_{4}\left(=D_{5}\right),
\end{aligned}
$$

and (using (6.10)) that

$$
\tilde{\alpha}_{j}^{i}=\left(\begin{array}{c|cccc}
6 & 1 & 2 & 2 & 1 \\
\hline 1 & -1 & 0 & 1 & 1 \\
2 & 0 & 0 & 1 & 1 \\
2 & 1 & 1 & 0 & 0 \\
1 & 1 & 1 & 0 & -1
\end{array}\right) .
$$

In (6.14) and (6.22) we have for example

$$
\begin{aligned}
\hat{\Gamma}\left(\mathrm{X}^{\circ}\right) & =1+\frac{1}{2} \sum C_{i}+3 C_{0}+\frac{360 \zeta(3)}{(2 \pi \mathbf{i})^{3}} p, \\
\hat{\xi}_{D_{0}} & =\mathcal{O}_{D_{0}}+\frac{1}{2} \mathcal{O}_{C_{1}}+\mathcal{O}_{C_{2}}+\mathcal{O}_{C_{3}}+\frac{1}{2} \mathcal{O}_{C_{4}}-4 \mathcal{O}_{p}, \\
-\hat{\xi}_{C_{j}} & =\mathcal{O}_{C_{j}}-\mathcal{O}_{p}=\mathcal{O}_{C_{j}}(-1)
\end{aligned}
$$

notice that $\hat{\xi}_{D_{0}}$ is not quite integral. One easily reads off the $N_{j}$ from $\tilde{\alpha}_{j}^{i}$ :

\begin{tabular}{c|c|c} 
& type & invariants \\
\hline$N_{0}$ & I & $\mathcal{O}_{p},\left\{\mathcal{O}_{C_{k}}\right\}_{k \neq 0}, \mathcal{O}_{D_{0}}$ \\
$N_{1}$ & IIa & $\mathcal{O}_{p},\left\{\mathcal{O}_{C_{k}}\right\}_{k \neq 1}, \mathcal{O}_{J_{1}}+\mathcal{O}_{J_{3}}, \mathcal{O}_{J_{1}}+\mathcal{O}_{J_{4}}, \mathcal{O}_{J_{2}}$ \\
$N_{2}$ & IIb & $\mathcal{O}_{p},\left\{\mathcal{O}_{C_{k}}\right\} k \neq 2, \mathcal{O}_{J_{1}}, \mathcal{O}_{J_{2}}, \mathcal{O}_{J_{3}}-\mathcal{O}_{J_{4}}$ \\
$N_{3}$ & IIb & $\mathcal{O}_{p},\left\{\mathcal{O}_{C_{k}}\right\}$ \\
$N_{4}$ & IIa & $\mathcal{O}_{p},\left\{\mathcal{O}_{C_{k}}\right\}_{k \neq 4}, \mathcal{O}_{J_{1}}+\mathcal{O}_{J_{4}}, \mathcal{O}_{J_{2}}, \mathcal{O}_{J_{3}}, \mathcal{O}_{J_{4}}, \mathcal{O}_{J_{3}}$
\end{tabular}

where $k$ runs from 0 to 4 .

This is in some sense incomplete, as the nonsimplicial nature of the Mori cone $\mathbb{R}_{\geq 0}\left\langle C_{1}, \ldots, C_{6}\right\rangle \subset H_{2}\left(\mathbb{P}_{\Delta^{\circ}}, \mathbb{R}\right)$ (and the dual "Kähler" cone in $H^{2}\left(\mathbb{P}_{\Delta^{\circ}}, \mathbb{R}\right)$ ) forces us to use all $6 \quad\left\{z_{i}\right\}$ to parametrize the singular 4dimensional domain of the B-model VHS, as described in $§ 5.6$. But this will not matter as we presently restrict to the Feynman locus, where $z_{i}=z_{i+3}(i=1,2,3)$ and $R_{0}^{(1)}=$ $R_{0}^{(4)}$ (cf. (5.19)-(5.18)), so that the mirror map zends $\underline{z} \mapsto R_{0}^{(1)}\left(J_{1}+J_{4}\right)+R_{0}^{(2)} J_{2}+R_{0}^{(3)} J_{3}$. This specialization therefore replaces Kähler by the 3 -dimensional simplicial "slice" $\mathbb{R}_{\geq 0}\left\langle J_{1}+J_{4}, J_{2}, J_{3}\right\rangle=\mathbb{R}_{\geq 0}\left\langle D_{2}+D_{3}, D_{3}+D_{4}, D_{1}+D_{2}\right\rangle$, and Mori by the 3-dimensional simplicial quotient $\mathbb{R}_{\geq 0}\left\langle\overline{C_{1}}, \overline{C_{2}}, \overline{C_{3}}\right\rangle$ in $\overline{H_{2}}:=H_{2}\left(\mathbb{P}_{\Delta^{\circ}}\right) /\left\langle C_{1}-C_{4}\right\rangle$. (Note that $\overline{C_{1}} \equiv \overline{C_{4}}$ 
$\Longrightarrow \overline{C_{2}}=\overline{C_{5}}$ and $\overline{C_{3}}=\overline{C_{6}}$; and that working modulo this equivalence, $\gamma\left(\hat{\xi}_{D_{0}}\right)$ becomes integral.) It also replaces $N_{1}$ and $N_{4}$ in the table by their sum $N_{1}+N_{4}$, which we compute to be (like $N_{2}$ and $N_{3}$ ) of type IIb, with invariants

$$
\mathcal{O}_{p}, \mathcal{O}_{C_{0}}, \mathcal{O}_{C_{1}}-\mathcal{O}_{C_{4}}, \mathcal{O}_{C_{2}}, \mathcal{O}_{C_{3}}, \mathcal{O}_{J_{1}}, \mathcal{O}_{J_{2}}-\mathcal{O}_{J_{3}}, \mathcal{O}_{J_{4}}
$$

We shall also have to define local Gromov-Witten invariants for classes $\ell_{1} \overline{C_{1}}+\ell_{2} \overline{C_{2}}+$ $\ell_{3} \overline{C_{3}} \in \overline{H_{2}}$, writing

$$
N_{\underline{\ell}}:=\sum_{k_{1}+k_{4}=\ell_{1}} \tilde{N}_{k_{1}, \ell_{2}, \ell_{3}, k_{4}} \in \mathbb{Q} .
$$

Now the statement of Theorem 6.1 for the sunset reads $(2 \pi \mathbf{i}) R_{1}=$

$$
=\left(R_{0}^{(1)}+R_{0}^{(2)}\right)\left(R_{0}^{(3)}+R_{0}^{(4)}\right)-\frac{1}{2}\left(R_{0}^{(1)}\right)^{2}-\frac{1}{2}\left(R_{0}^{(4)}\right)^{2}-\sum_{\underline{k} \neq \underline{0}}|\underline{k}| \tilde{N}_{\underline{k}} \underline{Q}^{\underline{k}},
$$

where $|\underline{k}|:=\sum_{i=1}^{4} k_{i}$. The Feynman specialization gives $R_{0}^{(1)}=R_{0}^{(4)}$ and $Q_{1}=Q_{4}$, and so writing $\underline{Q}^{\underline{\ell}}=Q_{1}^{\ell_{1}} Q_{2}^{\ell_{2}} Q^{\ell_{3}}$ and $|\underline{\ell}|=\sum_{i=1}^{3} \ell_{i}$, we have the

Corollary 6.3. - On the Feynman locus $\left(\cong\left(\Delta^{*}\right)^{3}\right)$ parametrizing the general-mass sunset family, we have

$$
(2 \pi \mathbf{i}) R_{1}=R_{0}^{(1)} R_{0}^{(2)}+R_{0}^{(2)} R_{0}^{(3)}+R_{0}^{(1)} R_{0}^{(3)}-\sum_{\underline{\ell} \in \mathbb{N}^{3} \backslash \underline{0}}|\underline{\ell}| N_{\underline{\ell}} \underline{Q}^{\underline{\ell}} .
$$

A computation of the local Gromov-Witten invariant is given in section 7.3.

\section{The multiparameter sunset integral}

In this section we use regulators (see §5.7) to derive the inhomogeneous Picard-Fuchs equation (Prop. 7.2) for the sunset integral, and also to relate it to the elliptic dilogarithm (Remark 7.5). This analysis complements the derivation of the Picard-Fuchs equation given in section 3.2 and the evaluation of the sunset integral in section 3.3. Using Corollary 6.3, we are able to derive an expression for the integral in terms of the local Gromov-Witten numbers, and to compute these numbers (Prop. 7.6ff).

7.1. Degeneration of the Yukawa coupling. — Let $\mathcal{B}$ denote the symplectic basis for the B-model $\mathbb{Q}$-local system given by applying $\Theta \circ \gamma$ to (6.21). According to $\S \S 6.2,6.4$ (esp. (6.13)) we find

$$
\begin{aligned}
{ }^{t}[\Omega]_{\mathcal{B}}= & \left(1, \tau_{0}, \ldots, \tau_{r-2}, \frac{\Phi_{0}^{\prime}}{(2 \pi \mathbf{i})^{3}}+\mathcal{O}(\underline{\tau}), \ldots, \frac{\Phi_{r-2}^{\prime}}{(2 \pi \mathbf{i})^{3}}+\mathcal{O}(\underline{\tau}),\right. \\
& \left.\frac{1}{(2 \pi \mathbf{i})^{3}}\left\{2 \Phi-\sum_{\ell=0}^{r-2} \tau_{\ell} \Phi_{\ell}^{\prime}\right\}+\mathcal{O}(\underline{\tau})\right)
\end{aligned}
$$


There are two ways to define Yukawa coupling: while (with $\delta_{z}:=z \partial_{z}$ )

$$
\tilde{Y}_{i j k}:=\int_{\mathrm{X}} \tilde{\Omega} \wedge \nabla_{\delta_{z_{i}} \delta_{z_{j}} \delta_{z_{k}}}^{3} \tilde{\Omega}
$$

makes sense "globally" (in $z_{0,}, \ldots, z_{k}$ ), we consider instead (referring to (6.28) for $\tau(z)$ )

$$
Y_{i j k}:=\int_{\mathrm{X}} \Omega \wedge \nabla_{\partial_{\tau_{i}} \partial_{\tau_{j}} \partial_{\tau_{k}}}^{3} \Omega
$$

which is defined "locally" about the large complex structure limit (in $q_{0}, \ldots, q_{k}$ ). Since $[Q]_{\mathcal{B}}$ is given by $(6.20),(7.3)$ is easily computed to be

$$
={ }^{t}[\Omega]_{\mathcal{B}}[Q]_{\mathcal{B}}[\Omega]_{\mathcal{B}}=\frac{1}{(2 \pi \mathbf{i})^{3}} \Phi_{i j k}^{(3)} .
$$

Motivated by the fact that the unique combination of first derivatives of $\Phi$ remaining finite in the $q_{0} \rightarrow 0\left(z_{0} \rightarrow 0\right)$ limit is $\Phi_{0}^{\prime}-\sum_{i=1}^{r-2} d_{i} \Phi_{i}^{\prime}$ (see the proof of Theorem 6.1), we look at

$$
\begin{aligned}
Y_{j k}^{\mathrm{loc}} & :=\lim _{q_{0} \rightarrow 0}\left(Y_{0 j k}-\sum_{i=1}^{r-2} d_{i} Y_{i j k}\right) \\
& =\alpha_{k}^{j}-\sum_{\underline{\kappa} \neq 0} \tilde{N}_{\underline{\kappa}}\left(\sum_{i=1}^{r-2} d_{i} \kappa_{i}\right) \kappa_{j} \kappa_{k} \underline{Q}^{\underline{\kappa}} \\
& =\frac{1}{(2 \pi \mathbf{i})^{2}} \Phi_{\text {loc }, j k}^{\prime \prime}=\frac{1}{2 \pi \mathbf{i}} \partial_{R_{0}^{(j)} R_{0}^{(k)}}^{2} R_{1} .
\end{aligned}
$$

To relate these to a Yukawa coupling on the elliptic curve family $\left\{E_{\underline{a}}\right\}$, write (cf. $(5.1),(5.3))$

$$
\omega_{\underline{a}}:=\frac{1}{2 \pi \mathbf{i}} \operatorname{Res}_{E_{\underline{a}}}\left(\frac{\frac{d x}{x} \wedge \frac{d y}{y}}{f_{\underline{a}}(x, y)}\right) \in \Omega^{1}\left(E_{\underline{a}}\right),
$$

and $\pi_{0}=\int_{\varphi_{0}} \omega, \pi_{1}=\int_{\varphi_{1}} \omega$. Now pass to the "diagonal slice" subfamily of [DK, §5.4], specializing $f_{\underline{a}}$ to $1-s \phi_{\ominus}$ where $\phi_{\ominus}(x, y)$ is a specific tempered $\Delta$-regular Laurent polynomial; by $[\mathrm{DK}, \S 5.4]$ we have $z_{i}(s) / s^{d_{i}}$ a root of $1(\forall i)$ and $R_{0}^{(1)} / d_{1} \equiv \cdots \equiv$ $R_{0}^{(r-2)} / d_{r-2} \equiv: R_{0} \bmod \mathbb{Q}(1)$. Moreover, one has $\delta_{s} R_{i}=\pi_{i}(i=1,2)$, and an easy computation reveals that

$$
\left.2 \pi \mathbf{i} \sum_{j, k} d_{j} d_{k} Y_{j k}^{\mathrm{loc}}\right|_{\Delta}=\partial_{R_{0}}^{2} R_{1}=\frac{\mathcal{Y}^{E}}{\pi_{0}^{3}},
$$

where

$$
\mathcal{Y}^{E}(s):=\int_{E_{s}} \omega_{s} \wedge \nabla_{\delta_{s}} \omega_{s}=\pi_{0} \delta_{s} \pi_{1}-\pi_{1} \delta_{s} \pi_{0}
$$

is the Yukawa coupling for $\left\{E_{s}\right\}:=\left\{E_{\{\underline{a}(s)}\right\}$. 
Remark 7.1. - In general, if $\mathrm{X}$ is replaced by a family of elliptically-fibered CalabiYau $(n+1)$-folds, and $E$ by a family of $(n-1)$-dimensional Calabi-Yaus $W$ with rank $n$ Picard-Fuchs equation along $\Delta$, a heuristic Hodge-theoretic argument shows that a $\left(z_{0} \rightarrow 0\right)$ limit of Yukawa couplings for X yields $\mathcal{Y}^{W} / \pi_{0}^{n+1}$ along $\Delta$.

For the rest of this section, we specialize to the sunset case. However, to treat the three-mass situation, we shall need to consider "arbitrary slices" of the Feynman locus, given by (the vanishing of)

$$
\begin{aligned}
f_{\underline{a}(s ; \underline{\xi})}(x, y) & :=f_{s}^{\ominus}(x, y):=1-s \phi_{\ominus}(x, y), \\
\phi_{\ominus}(x, y) & :=\left(1-x^{-1}-y^{-1}\right)\left(\xi_{3}^{2}-\xi_{2}^{2} x-\xi_{1}^{2} y\right) .
\end{aligned}
$$

(Note that $\phi_{\ominus}$ is no longer tempered.) We write $\mathcal{E}^{\xi} \stackrel{\varepsilon}{\rightarrow} \mathbb{P}_{s}^{1}$ for the family with fibers

$$
E_{s}^{\ominus}=\overline{\left\{f_{s}^{\ominus}(x, y)=0\right\}} \subset \mathbb{P}_{\Delta},
$$

and $\omega_{s}:=\omega_{\underline{a}}(s ; \underline{\xi})($ cf. $(7.5))$ for the section of $\varepsilon_{*} \omega_{\mathcal{E} / \mathbb{P}^{1}} \cong \mathcal{O}(1)$ with a simple zero at $s=\infty$. Note that this family is semistable.

The Yukawa coupling (with $\delta_{s}:=s \partial_{s}$ )

$$
Y_{\ominus}(s):=2 \pi \mathbf{i} \int_{E_{s}^{\ominus}} \omega_{s} \wedge \nabla_{\delta_{s}} \omega_{s} \in \mathbb{C}\left(\mathbb{P}^{1}\right)^{*} \cong \mathbb{C}(s)^{*}
$$

can be determined up to scale by the properties:

$-Y_{\odot}$ has a double zero at $\infty$;

$-Y_{\ominus}(0) \in \mathbb{C}^{*}$

- at other singular fibers, $Y_{\ominus}(s)$ has a simple pole;

- $Y_{\odot}(s)$ has a zero of order $m-1$ at branch points of order $m$ for the $J$-invariant $J(s)$; and

$-Y_{\odot}(0)=6$, by $(7.25)$ below.

This yields the function

$$
Y_{\ominus}(s)=\frac{2 \mu_{1} \mu_{2} \mu_{3} \mu_{4} s^{2}-4\left(\xi_{1}^{2}+\xi_{2}^{2}+\xi_{3}^{2}\right) s+6}{\prod_{i=1}^{4}\left(1-\mu_{i}^{2} s\right)},
$$

where $\mu_{1}=-\xi_{1}+\xi_{2}+\xi_{3}, \mu_{2}=\xi_{1}-\xi_{2}+\xi_{3}, \mu_{3}=\xi_{1}+\xi_{2}-\xi_{3}, \mu_{4}=\xi_{1}+$ $\xi_{2}+\xi_{3}$. This of course reproduces the expression for the Yukawa coupling in (3.50) derived in section 3.2.

We shall use this below to compute the local Gromov-Witten invariants $N_{\underline{\ell}}$, for simplicity of notation suppressing most " $\odot$ " subscripts in what follows.

7.2. Inhomogeneous equation for the sunset integral. - Continuing an analysis of the 1 -parameter family $\mathcal{E}_{\ominus} \stackrel{\varepsilon}{\rightarrow} \mathbb{P}^{1}$, we write as usual $\left\{\left\{\varphi_{0}^{(i)}\right\}_{i=1}^{6}, \varphi_{1}\right\} \subset \mathcal{K}_{E}$ (cf. 
\$5.4), and recall that on the Feynman locus, $\left\{\left\{\varphi_{0}^{(i)}\right\}_{i=1}^{3}, \varphi_{1}\right\}$ furnish a basis for $\overline{\mathcal{K}}_{E}$ (cf. $\S 5.6$ ). For the holomorphic period (about $s=0$ ), the usual residue computation yields

$$
\begin{aligned}
\pi_{0} & =\int_{\varphi_{0}} \omega=\int_{\varphi_{0}^{(i)}} \omega(i=1,2,3) \\
& =\sum_{m \geq 0} s^{m}\left(\sum_{|\underline{b}|=m} \underline{\xi}^{\underline{b}}\left(\begin{array}{c}
m \\
\underline{b}
\end{array}\right)^{2}\right)=: \sum_{m \geq 0} s^{m} \beta_{m},
\end{aligned}
$$

where $\left(\begin{array}{c}m \\ \underline{b}\end{array}\right)=\frac{m !}{b_{1} ! b_{2} ! b_{3} !}$ and the coefficients $\beta_{m}$ are generalized Apéry numbers. Writing $R=\frac{1}{2 \pi \mathbf{i}} R\{x, y\}=\frac{1}{2 \pi \mathbf{i}} \log (x) \frac{d y}{y}-\log (y) \delta_{T_{x}}$ for the regulator current on $E_{s}^{*}$, (5.20) gives for $i=1,2,3$

$$
R_{0}^{(i)}=\int_{\varphi_{0}^{(i)}} R=\log \left(\frac{-\xi_{i}^{2} s}{1-s \sum \xi_{i}^{2}}\right)+\mathcal{H}(s)
$$

where $\mathcal{H}$ is holomorphic (about $s=0$ ) and vanishes at $s=0$. Write $\mathcal{L}_{i}:=2 \log \left(\xi_{i}\right)$.

Interpreted as a 1-current on $\mathcal{E} \backslash E_{0}, R$ has coboundary

$$
d[R]=\frac{1}{2 \pi \mathbf{i}} \frac{d x}{x} \wedge \frac{d y}{y}-(2 \pi \mathbf{i}) \delta_{T_{x} \cap T_{y}}-\sum_{i=1}^{3} \log \left(\frac{m_{i}^{2}}{m_{i-1}^{2}}\right) \delta_{q_{i} \times \mathbb{P}^{1} \backslash\{0\}},
$$

where $\mathrm{q}_{1}, \mathrm{q}_{2}, \mathrm{q}_{3}, \mathrm{p}_{1}, \mathrm{p}_{2}, \mathrm{p}_{3}$ constitute the base locus of $\left\{E_{s}\right\}$ :

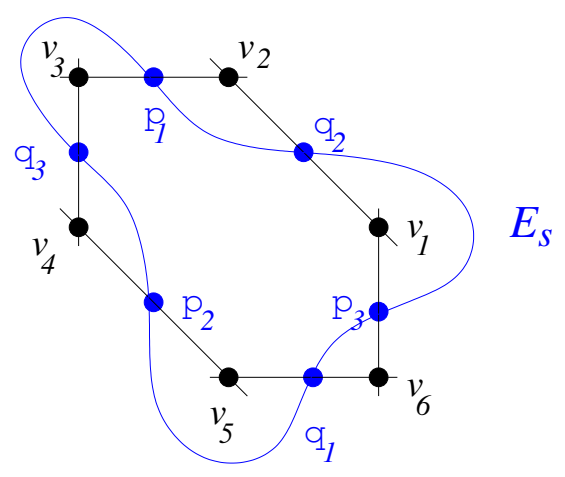

So locally over any small disk $U \subset \mathbb{P}^{1}$ avoiding the discriminant locus of $\varepsilon$, writing $\mathcal{P}^{i j} \rightarrow U$ for the 3 -chain with boundary $\mathrm{q}_{j} \times U-\mathrm{q}_{i} \times U$ (and fibers $P^{i j}$ ), we may construct the 1-current

$$
\hat{R}:=R-\left\{\mathcal{L}_{1} \delta_{\mathcal{P}^{23}}+\mathcal{L}_{2} \delta_{\mathcal{P}^{12}}+\mathcal{L}_{3} \delta_{\mathcal{P}^{31}}\right\}-(2 \pi \mathbf{i}) \delta_{\partial^{-1}\left(T_{x} \cap T_{y}\right)},
$$

which has $d[\hat{R}]=(2 \pi \mathbf{i})^{-1} d x / x \wedge d y / y$. Notice that its restriction to fibers $E_{s}$ is closed. ${ }^{(16)}$

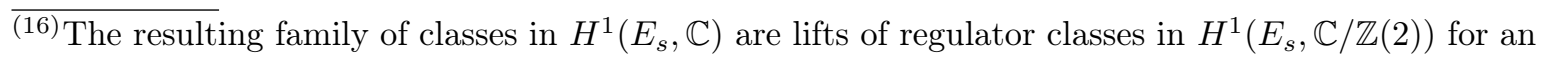
element of $C H^{2}\left(E_{s}, 2\right)$ precisely when the ratios $\xi_{i} / \xi_{i-1}$ are roots of unity, but we will not need this. 
For $\xi_{i}$ all 1 (equal masses) and $s \notin\left[0, \frac{1}{9}\right]$, we have $T_{x} \cap T_{y} \cap E_{s}=\emptyset$; moving the $\xi_{i}$ in a small neighborhood of $\underline{1}$, the "bad set" $\left[0, \frac{1}{9}\right]$ thickens slightly. Taking $U$ in the complement, we may ignore the last term of (7.11) for purposes of integrating over $\varphi_{0}^{(i)}$. Recall from [DK] that if $\varphi_{0}^{i}$ are the cycles that (at $\left.s=0\right)$ get pinched to $v_{i}$,

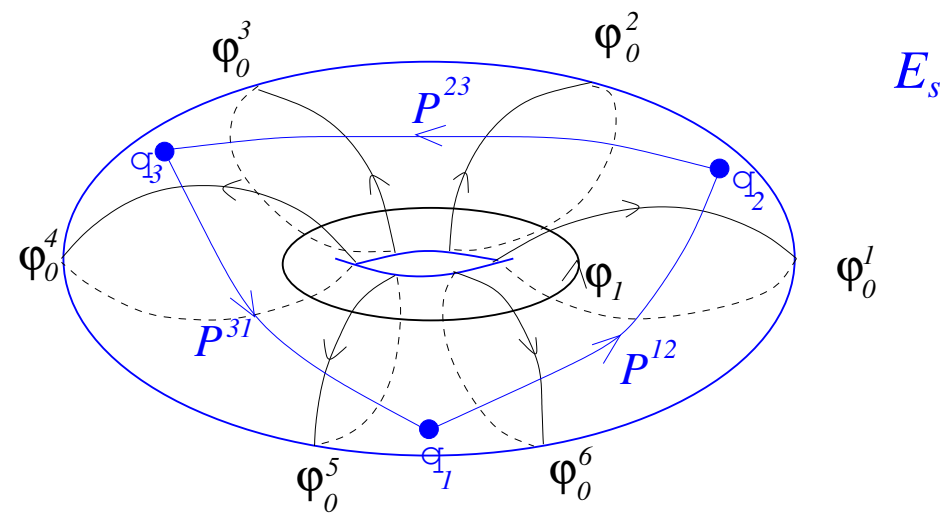

then

$$
\varphi_{0}^{(i)}=-\varphi_{0}^{i}+\varphi_{0}^{i+1}+\varphi_{0}^{i-1} .
$$

Together with $(7.11)$, the resulting intersection numbers ${ }^{(17)} \varphi_{0}^{(1)} \cdot P^{23}=\varphi_{0}^{(2)} \cdot P^{12}=$ $\varphi_{0}^{(3)} \cdot P^{31}=1$ (all others zero) yield

$$
\int_{\varphi_{0}^{(i)}} \hat{R}=R_{0}^{(i)}-\mathcal{L}_{i}=: \hat{R}_{0},
$$

which according to (7.9) (or the closedness of $\left.\hat{R}\right|_{E_{s}}$ ) is independent of $i$. As suggested by the picture, we can also choose the $P^{i j}$ to avoid $\varphi_{1}$, and so

$$
\hat{R}_{1}:=\int_{\varphi_{1}} \hat{R}=R_{1} \text {. }
$$

Next consider the interior product of $d[\hat{R}]$ with a lift of $s \frac{d}{d s}$ : working over $U$,

$$
\begin{aligned}
2 \pi \mathbf{i} \cdot d[\hat{R}]\lrcorner \widetilde{\frac{d}{d s}} & \left.=\frac{d x}{x} \wedge \frac{d y}{y}\right\lrcorner \widetilde{s \frac{d}{d s}} \\
& \left.=-\operatorname{Res}_{\mathcal{E}}\left(\frac{d x}{x} \wedge \frac{d y}{y} \wedge \operatorname{dlog}\left(s^{-1}-\phi_{\ominus}\right)\right)\right\lrcorner \widetilde{s \frac{d}{d s}}
\end{aligned}
$$

restricts on fibers to

$$
\left.-\operatorname{Res}_{E_{s}}\left(\frac{d x}{x} \wedge \frac{d y}{y} \wedge \frac{-d s / s^{2}}{s^{-1}-\phi_{\ominus}}\right\lrcorner s \frac{d}{d s}\right)=\operatorname{Res}_{E_{s}}\left(\frac{\frac{d x}{x} \wedge \frac{d y}{y}}{1-s \phi_{\ominus}}\right)=(2 \pi \mathbf{i}) \omega_{s} .
$$

It follows that

$$
\nabla_{\delta_{s}}\left[\left.\hat{R}\right|_{E_{s}}\right]=\left[\omega_{s}\right]
$$

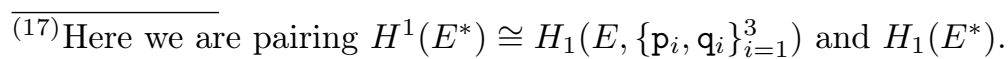


which along with (7.12) implies that

$$
R_{0}^{(i)}-\mathcal{L}_{i}\left(=\hat{R}_{0}\right)=\log (-s)+\sum_{m>0} \frac{s^{m}}{m} \beta_{m}
$$

up to an additive constant. This constant is obviously zero by (7.9).

Now recall that the Feynman integral is given by $\mathcal{I}_{\ominus}(s):=-s V_{\ominus}(s)$

$$
V_{\ominus}(s)=\int_{T_{x} \cap T_{y}} \frac{\frac{d x}{x} \wedge \frac{d y}{y}}{1-s \phi_{\ominus}}=: \int_{T_{x} \cap T_{y}} \hat{\omega}_{s} .
$$

Writing $\imath^{s}: E_{s} \hookrightarrow \mathbb{P}_{\Delta}$, and $R=\frac{1}{2 \pi \mathbf{i}} R\{x, y\}$ as above, we note that $d\left[\hat{\omega}_{s}\right]=(2 \pi \mathbf{i})^{2} \imath_{*}^{s} \omega_{s}$ as a current, and that $\left(\right.$ on $\left.\mathbb{P}_{\Delta}\right)$

$$
d\left[\frac{1}{2 \pi \mathbf{i}} R\right]=\frac{1}{(2 \pi \mathbf{i})^{2}} \frac{d x}{x} \wedge \frac{d y}{y}-\delta_{T_{x} \cap T_{y}}+\left\{\text { residue terms supported on } \mathbb{D}_{\Delta}\right\} .
$$

Using integration by parts (for currents), we get that (7.16) becomes

$$
\frac{1}{2 \pi \mathbf{i}} \int_{\mathbb{P}_{\Delta}} R \wedge d\left[\hat{\omega}_{s}\right]=2 \pi \mathbf{i} \int_{\mathbb{P}_{\Delta}} R \wedge i_{*}^{s} \omega_{s}=\left.2 \pi \mathbf{i} \int_{E_{s}} R\right|_{E_{s}} \wedge \omega_{s}
$$

(Note that (7.17) is not a truncated higher normal function in the sense of [DK], and neither is $\left\langle\left.\hat{R}\right|_{E_{s}}, \omega_{s}\right\rangle$ in (7.18) below.) Since $\partial^{-1}\left(T_{x} \cap T_{y}\right)$ in (7.11) avoids $\varepsilon^{-1}(U)$ (and $s \in U)$, we conclude that ${ }^{(18)}$

$$
V_{\ominus}(s)=\left\langle\left.\hat{R}\right|_{E_{s}}, \tilde{\omega}_{s}\right\rangle+\sum_{i=1}^{3} \mathcal{L} \tilde{\pi}_{1}^{(i)}(s ; \underline{\xi}),
$$

where $\tilde{\omega}_{s}:=(2 \pi \mathbf{i}) \omega_{s}$, and

$$
\tilde{\pi}_{1}^{(1)}:=\int_{\mathrm{q}_{2}}^{\mathrm{q}_{3}} \tilde{\omega}_{s}, \tilde{\pi}_{1}^{(2)}:=\int_{\mathrm{q}_{1}}^{\mathrm{q}_{2}} \tilde{\omega}_{s}, \tilde{\pi}_{1}^{(3)}:=\int_{\mathrm{q}_{3}}^{\mathrm{q}_{1}} \tilde{\omega}_{s} .
$$

Note that the $\left\{q_{i}\right\}$ depend on $\underline{\xi}$, and that $\sum_{j=1}^{3} \tilde{\pi}_{1}^{(j)}=\tilde{\pi}_{1}\left(=2 \pi \mathbf{i} \pi_{1}\right)$.

Let $\theta=\delta_{s}^{2}+q_{1}(s) \delta_{s}+q_{0}(s)$ be the Picard-Fuchs operator associated to $\left\{\omega_{s}\right\}$, so that $\nabla_{\delta_{s}}^{2}+f(s) \nabla_{\delta_{s}}+g(s)$ kills $\left[\omega_{s}\right]$. Using (7.14) and (7.6), we find $\delta_{s}\langle\hat{R}, \omega\rangle=\left\langle\hat{R}, \nabla_{\delta_{s}} \omega\right\rangle$ and $\delta_{s}^{2}\langle\hat{R}, \omega\rangle=(2 \pi \mathbf{i})^{-1} Y_{\ominus}(s)+\left\langle\hat{R}, \nabla_{\delta_{s}}^{2} \omega\right\rangle$, which leads at once to the inhomogeneous Picard-Fuchs equation:

Proposition 7.2. - We have

$$
\theta\left(V_{\ominus}(s)\right)=\theta\left(-\frac{1}{s} \mathcal{I}_{\ominus}(s)\right)=Y_{\ominus}(s)+\sum_{j=1}^{3} \log \left(\xi_{j}^{2}\right) \nu_{j}(s),
$$

$\overline{{ }^{(18)} \text { Of course }}\langle\hat{R}, \omega\rangle$ means $\int_{E_{s}} \hat{R} \wedge \omega$; we write it this way to emphasize that fact that two cohomology classes are being paired. 
where

$$
\nu_{i}(s):=\theta\left(\tilde{\pi}_{1}^{(i)}(s ; \underline{\xi})\right)
$$

satisfy $\sum_{i=1}^{3} \nu_{i}=0$.

Remark 7.3. - (i) The functions in (7.21) belong to $\overline{\mathbb{Q}}(s)^{*}$, since the partial elliptic integrals in (7.19) are the normal functions associated to globally well-defined algebraic 0-cycles $\left[\mathbf{q}_{j+1}\right]-\left[\mathbf{q}_{j}\right]$ on the family $\left\{E_{s}\right\}$, and the section $\left\{\tilde{\omega}_{s}\right\}$ of the relative canonical bundle is defined over $\overline{\mathbb{Q}}$.

(ii) The right-hand-side of (7.20) only depends on $s$ and the mass ratios, since this is true for $\nu_{j}$ and $Y_{\odot}$; and we have $\sum_{j=1}^{3} \mathcal{L}_{j} \nu_{j}=\log \left(m_{2}^{2} / m_{3}^{2}\right) \nu_{1}+\log \left(m_{1}^{2} / m_{3}^{2}\right) \nu_{2}$.

Remark 7.4. - The coefficients $q_{1}(s)$ and $q_{0}(s)$ are respectively given in (3.40) and (3.45). An explicit expression for the $\nu_{i}(s)$ in some coordinate system is given in section (3.49). In particular $\prod_{i=1}^{4}\left(s \mu_{i}^{2}-1\right)\left(s^{2} \prod_{i=1}^{4} \mu_{i}-2 s\left(\xi_{1}^{2}+\xi_{2}^{2}+\xi_{3}^{2}\right)+3\right) \nu_{i}(s)=12 s c_{i}(s)$ with $c_{1}(s)$ $\begin{array}{llll}\text { given } & \text { in } & (3.51) & \text { and }\end{array}$ in $(3.52)$.

Remark 7.5. - One can also relate (7.17) directly to the elliptic dilogarithm. Noting that up to coboundary $-\left.(2 \pi \mathbf{i}) R\right|_{E_{s}} \equiv \log (y) \frac{d x}{x}-(2 \pi \mathbf{i}) \log (x) \delta_{T_{y}}$, we get

$$
\mathcal{I}_{\ominus}(s)=-s \int_{T_{y}\left(\cap E_{s}\right)} \log (x) \tilde{\omega}_{s}
$$

Recalling that $\partial T_{y}=(y)$, this connects at once to the expression for the sunset integral in (4.27) hence (3.79).

7.3. On the local Gromov-Witten numbers. - Turning to the numbers $N_{\underline{\ell}}=$ $N_{\ell_{1}, \ell_{2}, \ell_{3}}$ (cf. (6.32)), note first that symmetries of $\mathbb{P}_{\Delta}$ immediately imply that for any $\sigma \in S_{3}$,

$$
N_{\underline{\ell}}=N_{\sigma(\underline{\ell})}
$$

We also know that

$$
N_{100}+N_{010}+N_{001}=6,
$$

as this is the number of "anticanonical-degree-one" rational curves on $\mathbb{P}_{\Delta}$ (the six toric boundary components).

The symmetries also force the prepotential $\Phi_{l o c}=(2 \pi \mathbf{i}) R_{1}$ to be symmetric in the $\tau_{i}=(2 \pi \mathbf{i}) R_{0}^{(i)}(i=1,2,3)$. Indeed, this is already recorded in (6.33), which combined with (7.12) and (7.13) becomes

$$
(2 \pi \mathbf{i}) \hat{R}_{1}=3 \hat{R}_{0}^{2}+2\left(\sum \mathcal{L}_{i}\right) \hat{R}_{0}-\sum_{\ell>0} \ell N_{\ell} \hat{Q}^{\ell}
$$


where $\hat{Q}=e^{\hat{R}_{0}}$, and

$$
N_{\ell}:=\sum_{|\underline{\ell}|=\ell} N_{\underline{\ell}} \underline{\xi}^{2 \underline{\ell}}
$$

But since $\nabla_{\delta_{s}}[\hat{R}]=[\omega]$, we have immediately $\delta_{s} \hat{R}_{1}=\pi_{1}$ and $\delta_{s} \hat{R}_{0}=\pi_{0}$, so that

$$
(2 \pi \mathbf{i}) \frac{\partial^{2} \hat{R}_{1}}{\partial \hat{R}_{0}^{2}}=(2 \pi \mathbf{i}) \frac{\partial}{\partial \hat{R}_{0}} \frac{\pi_{1}}{\pi_{0}}=\frac{Y_{\ominus}}{\pi_{0}^{3}} .
$$

Putting together the expressions of the Yukawa coupling (7.7), the period $\pi_{0}$ and the coefficients $\beta_{m}$ in (7.8), for $\hat{R}_{0}$ in (7.15), the expansion of $\hat{R}_{1}$ in (7.23) and (7.25) now yields the

Proposition 7.6. - In a neighborhood of $s=0(\hat{Q}=0)$, we have

$$
6-\sum_{\ell>0} \ell^{3} N_{\ell} \hat{Q}^{\ell}=\frac{6-4\left(\xi_{1}^{2}+\xi_{2}^{2}+\xi_{3}^{2}\right) s+2 \mu_{1} \mu_{2} \mu_{3} \mu_{4} s^{2}}{\left(1+\sum_{m>0} \beta_{m} s^{m}\right)^{3} \prod_{i=1}^{4}\left(1-\mu_{i}^{2} s\right)},
$$

where $\hat{Q}=-s \exp \left\{\sum_{m>0} \frac{\beta_{m} s^{m}}{m}\right\}$.

We may use Proposition 7.6 to recover the $N_{\underline{\ell}}$, as well as the local "instanton numbers" $n_{\underline{\ell}}$ defined by the Aspinwall-Morrison formula [AM, Vo]

\begin{tabular}{|c|c|c|c|c|c|c|c|c|c|}
\hline$\underline{\ell}$ & $(100)$ & $\begin{array}{c}k>0 \\
(k 00)\end{array}$ & (110) & $(210)$ & (111) & $(310)$ & $(220)$ & $(211)$ & $(221)$ \\
\hline$\overline{\bar{N} N_{\ell}}$ & 2 & $2 / k^{3}$ & $\overline{-2}$ & $\overline{0}$ & 6 & $\overline{0}$ & $-1 / 4$ & -4 & 10 \\
\hline$n_{\underline{\ell}}$ & 2 & 0 & -2 & 0 & 6 & 0 & 0 & -4 & 10 \\
\hline$\underline{\ell}$ & $(410)$ & $(320)$ & $(311)$ & $(510)$ & $(420)$ & (411) & $(330)$ & $(321)$ & $(222)$ \\
\hline$\overline{N_{\underline{\ell}}}$ & 0 & 0 & $\overline{0}$ & $\overline{0}$ & $\overline{0}$ & 0 & $-2 / 27$ & -1 & $-189 / 4$ \\
\hline$n_{\ell}$ & 0 & 0 & 0 & 0 & 0 & 0 & 0 & -1 & -48 \\
\hline
\end{tabular}

$$
N_{\ell_{1}, \ell_{2}, \ell_{3}}=\sum_{d \mid \ell_{1}, \ell_{2}, \ell_{3}} \frac{1}{d^{3}} n_{\frac{\ell_{1}}{d}, \frac{\ell_{2}}{d}, \frac{\ell_{3}}{d}} .
$$

As far as we computed, the latter are integers:

Finally, we note that the Gromov-Witten invariants appear directly in the Feynman integral, as follows. Write $-s^{-1} \mathcal{I}_{\ominus}=-s^{-1} \tilde{\mathcal{I}}_{\ominus}+\sum_{i=1}^{3} \mathcal{L}_{i} \tilde{\pi}_{1}^{(i)}$, and apply $\partial_{\hat{R}_{0}}$ to (7.23) to have

$$
(2 \pi \mathbf{i}) \frac{\pi_{1}}{\pi_{0}}=6 \hat{R}_{0}+2 \sum_{i=1}^{3} \mathcal{L}_{i}-\sum_{\ell>0} \ell^{2} N_{\ell} \hat{Q}^{\ell}
$$


The contribution $\tilde{\mathcal{I}}_{\ominus}$ to the Feynman integral read

$$
\begin{aligned}
-s^{-1} \tilde{\mathcal{I}}_{\ominus}(s) & =2 \pi \mathbf{i}\langle\hat{R}, \omega\rangle=2 \pi \mathbf{i}\left(\pi_{1} \hat{R}_{0}-\pi_{0} \hat{R}_{1}\right) \\
& =\pi_{0}\left(2 \pi \mathbf{i} \frac{\pi_{1}}{\pi_{0}} \hat{R}_{0}-2 \pi \mathbf{i} \hat{R}_{1}\right),
\end{aligned}
$$

which using $\pi_{1} / \pi_{0}=\delta_{s} \hat{R}_{1} / \delta_{s} \hat{R}_{0}=\partial \hat{R}_{1} / \partial R_{0}$ leads to the expression as a Legendre transform of $\hat{R}_{1}$

$$
\tilde{\mathcal{I}}_{\ominus}(s)=-s 2 \pi \mathbf{i} \pi_{0}\left(\frac{\partial \hat{R}_{1}}{\partial \hat{R}_{0}} \hat{R}_{0}-\hat{R}_{1}\right) .
$$

This expression has the expansion

$$
\tilde{\mathcal{I}}_{\ominus}(s)=-s \pi_{0}\left\{3 \hat{R}_{0}^{2}+\sum_{\ell>0} \ell\left(1-\ell \hat{R}_{0}\right) N_{\ell} \hat{Q}^{\ell}\right\},
$$

The occurrence of the Gromov-Witten numbers in this Feynman integral seems to be novel.

\subsection{The local Gromov-Witten numbers in the}

all equal masses case. - In this subsection we compute the local Gromov-Witten invariants for the all equal masses case. The family of elliptic curves $\mathcal{E}_{\ominus}:=\{x y z-$ $\left.s(x+y+z)(x y+x z+y z)=0 \mid(x, y, z) \in \mathbb{P}^{2}\right\}$ defines a pencil of elliptic curves in $\mathbb{P}^{2}$ corresponding to a modular family of elliptic curves $f: \mathcal{E}_{\ominus} \rightarrow X_{1}(6)=\{\tau \in \mathbb{C} \mid \Im m(\tau)>$ $0\} / \Gamma_{1}(6)$ (see $\left.[\mathrm{BV}]\right)$.

7.4.1. The local Gromov-Witten numbers. - In this case Proposition 7.6 applied to the case $\xi_{1}=\xi_{2}=\xi_{3}=1$ implies that

$$
6-\sum_{\ell \geq 1} \ell^{3} N_{\ell} \hat{Q}^{\ell}=\frac{6}{(9 s-1)(s-1) \pi_{0}^{3}},
$$

where the holomorphic period (about $s=0)$ of $(7.8)$ reads

$$
\pi_{0}=\sum_{\ell \geq 0} s^{\ell} \sum_{p_{1}+p_{2}+p_{3}=\ell}\left(\frac{\ell !}{p_{1} ! p_{2} ! p_{3} !}\right)^{2} .
$$

and $\hat{Q}=\exp \left(\hat{R}_{0}\right)$ where $\hat{R}_{0}$ in (7.12) satisfies $s d R_{0} / d s=\pi_{0}$ and reads

$$
R_{0}=\mathbf{i} \pi+\log s+\sum_{\ell>0} \frac{s^{\ell}}{\ell} \sum_{p_{1}+p_{2}+p_{3}=\ell}\left(\frac{\ell !}{p_{1} ! p_{2} ! p_{3} !}\right)^{2} .
$$

Taking for $s$ the Hauptmodul used in [BV]

$$
s_{\odot}(q)^{-1}=9+72 \frac{\eta\left(q^{2}\right)}{\eta\left(q^{3}\right)}\left(\frac{\eta\left(q^{6}\right)}{\eta(q)}\right)^{5}
$$


we have

$$
\pi_{0}(q)=\frac{1}{4} \frac{\theta_{2}^{3}(q)}{\theta_{2}\left(q^{3}\right)}
$$

and

$$
\hat{R}_{0}(q)=\mathbf{i} \pi+\log q-\sum_{n \geq 1}(-1)^{n-1}\left(\frac{-3}{n}\right) n \operatorname{Li}_{1}\left(q^{n}\right),
$$

where $\left(\frac{-3}{n}\right)=0,1,-1$ for $n \equiv 0,1,2 \bmod 3$.

From (7.29) we compute the local Gromov-Witten numbers $N_{\ell}$

$$
\begin{aligned}
N_{\ell} / 6= & 1,-\frac{7}{8}, \frac{28}{27},-\frac{135}{64}, \frac{626}{125},-\frac{751}{54}, \frac{14407}{343},-\frac{69767}{512}, \frac{339013}{729},-\frac{827191}{500}, \\
& \frac{8096474}{1331},-\frac{367837}{16}, \frac{195328680}{2197},-\frac{137447647}{392}, \frac{4746482528}{3375}, \\
& -\frac{23447146631}{4096}, \frac{115962310342}{4913},-\frac{574107546859}{5832}, \frac{2844914597656}{6859}, \\
& -\frac{1410921149451}{800}, \frac{10003681368433}{1323}, \ldots
\end{aligned}
$$

or introducing $n_{\ell}$ the virtual number of degree $\ell$ rational curves using the AspinwallMorrison multiple cover formula [AM, Vo]

$$
N_{\ell}=\sum_{d \mid \ell} \frac{1}{d^{3}} n_{\frac{\ell}{d}}
$$

we have

$$
\begin{aligned}
n_{k} / 6= & 1,-1,1,-2,5,-14,42,-136,465,-1655,6083,-22988, \\
& 88907,-350637,1406365,-5724384,23603157,-98440995, \\
& 414771045,-1763651230,7561361577,-32661478080, \\
& 142046490441,-621629198960,2736004885450, \\
& -12105740577346,53824690388016, \ldots
\end{aligned}
$$

7.4.2. Comparing the two expansions. - We will show how to relate the $q$ and $Q$ expansions using a $\Gamma_{1}(6)$ modular transformation. In the all equal masses case the sunset integral was given by $[\mathrm{BV}]$

$$
\mathcal{I}_{\ominus}(s) \equiv \frac{\varpi_{r}}{\pi} E_{\ominus}(q) \quad \text { mod period }
$$

with $E_{\ominus}(q)$ given in (3.75). The expression is modulo periods of the elliptic curve, and $\varpi_{r}$ is the real period on the real axis $s>\left(\xi_{1}+\xi_{2}+\xi_{3}\right)^{-2}$ given in (3.14).

The all equal masses case the sunset integral is equal to $\tilde{\mathcal{I}}_{\ominus}$ in $(7.28)$

$$
\mathcal{I}_{\ominus}(s) \equiv-s\left(\pi_{0} \hat{R}_{1}-\pi_{1} \hat{R}_{0}\right) \quad \bmod \text { period }
$$


where $\pi_{0}$ is the holomorphic period around $s=0$ and $\pi_{1}$ is the other non-holomorphic period in (7.19), and $\hat{R}_{1}$ is such that $s d \hat{R}_{1} / d s=\pi_{1}$ of (7.23). The modular transformations $\tau \rightarrow-1 /(6 \tau)$ maps the periods as

$$
\begin{aligned}
\varpi_{r}(-1 /(6 \tau)) & =-6 s_{\ominus}(\tau)(2 \mathbf{i} \pi \tau) \pi_{0}(\tau) ; \\
\pi_{1}(-1 / 6 \tau) & =\frac{3 \tau-1}{6} s_{\ominus}(\tau)^{-1} \varpi_{r}(\tau) .
\end{aligned}
$$

The same modular transformation applied to the sunset integral leads to the relation between the elliptic dilogarithm $E_{\ominus}(q)$ and the regulator period

$$
36 \mathbf{i} \tau E_{\ominus}(-1 /(6 \tau))=\pi^{2}+3 \mathbf{i} \pi \log (-q)+3\left(\hat{R}_{1}(\tau)-\frac{\partial \hat{R}_{1}}{\partial \hat{R}_{0}} \hat{R}_{0}\right) .
$$

This shows that $E_{\ominus}(q)$ is the Legendre transform of $\hat{R}_{1}(q)$ as expected from the general different masses case in (7.27). Using the $q$-expansion given above and using that $\partial \hat{R}_{1} / \partial \hat{R}_{0}=\log (-q)$ we have

$$
\begin{aligned}
\hat{R}_{1}(q)-\frac{\partial \hat{R}_{1}}{\partial \hat{R}_{0}} \hat{R}_{0}= & -\frac{1}{2} \log (-q)^{2} \\
& +\sum_{n \geq 1}\left(\sum_{d \mid n}(-1)^{d} d^{2}\left(\frac{-3}{d}\right)\right) \operatorname{Li}_{2}\left(q^{n}\right) .
\end{aligned}
$$

\section{PART IV \\ APPENDICES}

\section{A}

\section{Theta functions}

In this appendix we recall standard results on Jacobi theta functions that are used in the text. We use the notation $q=e^{2 \pi \mathbf{i} \tau}$ with $\tau$ the period ratio chosen to lie in the upper-half-plane, and $x \in \mathbb{C}^{\times} / q^{\mathbb{Z}}$

$$
\theta_{1}(x):=\frac{q^{\frac{1}{8}}}{\mathbf{i}}\left(x^{\frac{1}{2}}-x^{-\frac{1}{2}}\right) \prod_{n=1}^{\infty}\left(1-q^{n}\right)\left(1-q^{n} x\right)\left(1-q^{n} / x\right),
$$

and

$$
\theta_{2}(x):=q^{\frac{1}{8}}\left(x^{\frac{1}{2}}+x^{-\frac{1}{2}}\right) \prod_{n=1}^{\infty}\left(1-q^{n}\right)\left(1+q^{n} x\right)\left(1+q^{n} / x\right),
$$


and

$$
\theta_{3}(x):=\prod_{n=1}^{\infty}\left(1-q^{n}\right)\left(1+q^{n-1 / 2} x\right)\left(1+q^{n-1 / 2} / x\right)
$$

and finally

$$
\theta_{4}(x):=\prod_{n=1}^{\infty}\left(1-q^{n}\right)\left(1-q^{n-1 / 2} x\right)\left(1-q^{n-1 / 2} / x\right) .
$$

We will use the shorthand notation $\theta_{a}:=\theta_{a}(1)$ for $a=2,3,4$, or $\theta_{\alpha}(q)$ when needed. A particular case of the Jacobi identity is

$$
\theta_{3}^{2}(v) \theta_{3}^{2}(u)+\theta_{1}^{2}(v) \theta_{1}^{2}(u)=\theta_{2}^{2}(v) \theta_{2}^{2}(u)+\theta_{4}^{2}(v) \theta_{4}^{2}(u)
$$

Applying this identity for $v=\exp (\mathbf{i} \pi(a+b \tau))$ with $a, b \in\{0,1\}$ one obtains the following quadratic relations satisfied by the theta functions

$$
\left(\begin{array}{cccc}
0 & \theta_{2}^{2} & -\theta_{3}^{2} & \theta_{4}^{2} \\
\theta_{2}^{2} & 0 & \theta_{4}^{2} & -\theta_{3}^{2} \\
\theta_{3}^{2} & \theta_{4}^{2} & 0 & -\theta_{2}^{2} \\
\theta_{4}^{2} & \theta_{3}^{2} & -\theta_{2}^{2} & 0
\end{array}\right)\left(\begin{array}{c}
\theta_{1}^{2}(u) \\
\theta_{2}^{2}(u) \\
\theta_{3}^{2}(u) \\
\theta_{4}^{2}(u)
\end{array}\right)=\left(\begin{array}{l}
0 \\
0 \\
0 \\
0
\end{array}\right)
$$

\section{B}

\section{The coefficients of the Picard-Fuchs equation}

In this appendix we give the explicit expressions for the coefficients of the homogeneous polynomials used when deriving the sunset Picard-Fuchs equation.

B.1. The coefficients $C_{x}, C_{y}$ and $C_{z}$ - The coefficients $C_{x}, C_{y}$ and $C_{z}$ are homogeneous polynomials of degree 4 in $(x, y, z)$ of the form

$$
\begin{aligned}
& C_{x}=x y^{2} z C_{x}^{1,2,1}+x^{2} z^{2} C_{x}^{2,0,2}+x^{2} y z C_{x}^{2,1,1}+x^{3} z C_{x}^{3,0,1}, \\
& C_{y}=x y z^{2} C_{y}^{1,1,2}+x y^{2} z C_{y}^{1,2,1}+x^{2} z^{2} C_{y}^{2,0,2}+x^{2} y z C_{y}^{2,1,1}, \\
& C_{z}=x z^{3} C_{z}^{1,0,3}+x y z^{2} C_{z}^{1,1,2}+x y^{2} z C_{z}^{1,2,1}+x^{2} z^{2} C_{z}^{2,0,2}+x^{2} y z C_{z}^{2,1,1} .
\end{aligned}
$$


Their detailed expressions are given by for $C_{x}$

$$
\begin{aligned}
& 6 \prod_{i=1}^{4}\left(s \mu_{i}^{2}-1\right) C_{x} \\
&= s x z\left(m_{1}^{2} x(9 x+20 y)+3 m_{2}^{2} y(6 x+y)+2 m_{3}^{2} x(10 y-3 z)\right) \\
&+ s^{4} x z\left(m_{1}^{4}-2 m_{1}^{2}\left(m_{2}^{2}+m_{3}^{2}\right)+\left(m_{2}^{2}-m_{3}^{2}\right)^{2}\right) \\
& \times\left(m_{1}^{4} x(x+y)+m_{1}^{2}\left(m_{2}^{2}\left(5 x^{2}+8 x y+3 y^{2}\right)-m_{3}^{2} x(5 x+2(y+z))\right)\right. \\
&+\left.\left(m_{2}^{2}-m_{3}^{2}\right)\left(3 m_{2}^{2} y(x+y)-m_{3}^{2} x(y-2 z)\right)\right) \\
&-s^{2} x z\left(m_{1}^{4} x(17 x+18 y)\right. \\
&+m_{1}^{2}\left(m_{2}^{2}\left(13 x^{2}+46 x y+3 y^{2}\right)+3 m_{3}^{2} x(-3 x+4 y+2 z)\right) \\
&\left.+3 m_{2}^{4} y(4 x+y)+m_{2}^{2} m_{3}^{2}\left(10 x y-14 x z+9 y^{2}\right)+2 m_{3}^{4} x(9 y-5 z)\right) \\
&+s^{3} x z\left(m_{1}^{6} x(7 x+4 y)\right. \\
&+m_{1}^{4}\left(m_{2}^{2}\left(18 x^{2}+22 x y-3 y^{2}\right)-2 m_{3}^{2} x(5 x+2 y-7 z)\right) \\
&-m_{1}^{2}\left(m_{2}^{4}\left(x^{2}-24 x y-30 y^{2}\right)\right. \\
& \quad+2 m_{2}^{2} m_{3}^{2}\left(-7 x^{2}-22 x y+2 x z+3 y^{2}\right) \\
& \quad\left.+m_{3}^{4} x(13 x+4 y+28 z)\right) \\
&-\left(m_{2}^{2}-m_{3}^{2}\right)\left(m_{2}^{4} y(2 x+3 y)+m_{2}^{2} m_{3}^{2}\left(2 x(y+5 z)+9 y^{2}\right)\right. \\
&\left.\left.\quad+2 m_{3}^{4} x(2 y-z)\right)\right)-7 x^{2} y z
\end{aligned}
$$


for $C_{y}$

$$
\begin{aligned}
& 3 \prod_{i=1}^{4}\left(s \mu_{i}^{2}-1\right) C_{y} \\
& =-2 s x y z\left(m_{1}^{2}(3 x+2 y)+3 m_{2}^{2} y+m_{3}^{2}(2 y+3 z)\right) \\
& -2 s^{4} x z\left(m_{1}^{4}-2 m_{1}^{2}\left(m_{2}^{2}+m_{3}^{2}\right)+\left(m_{2}^{2}-m_{3}^{2}\right)^{2}\right) \\
& \quad \times\left(m_{1}^{4} y(x+y)-m_{1}^{2}\left(m_{2}^{2} y(x+y)+m_{3}^{2}\left(5 x y+6 x z+2 y^{2}+5 y z\right)\right)\right. \\
& \left.\quad+m_{3}^{2} y\left(m_{3}^{2}-m_{2}^{2}\right)(y+z)\right) \\
& +2 s^{2} x z\left(5 m_{1}^{4} x y+m_{1}^{2}\left(m_{2}^{2} y(7 x+y)-3 m_{3}^{2}(7 x y+6 x z+7 y z)\right)\right. \\
& \left.\quad+y\left(3 m_{2}^{4} y+m_{2}^{2} m_{3}^{2}(y+7 z)+5 m_{3}^{4} z\right)\right) \\
& -2 s^{3} x z\left(m_{1}^{6} y(x-2 y)+m_{1}^{4}\left(m_{2}^{2} y(y-6 x)+m_{3}^{2}(2 x(y-6 z)+y(2 y-19 z))\right)\right. \\
& \quad+m_{1}^{2}\left(5 m_{2}^{4} x y-2 m_{2}^{2} m_{3}^{2}(x(5 y+6 z)+5 y(y+z))\right. \\
& \left.\quad+m_{3}^{4}(2 y(y+z)-x(19 y+12 z))\right) \\
& \left.\quad+y\left(m_{2}^{6} y+5 m_{2}^{4} m_{3}^{2} z+m_{2}^{2} m_{3}^{4}(y-6 z)+m_{3}^{6}(z-2 y)\right)\right)+2 x y^{2} z,
\end{aligned}
$$


and for $C_{z}$

$$
\begin{aligned}
6 \prod_{i=1}^{4}\left(s \mu_{i}^{2}-1\right) C_{z} \\
=s x z\left(-2 m_{1}^{2}(6 x y+9 x z-y z)-3 m_{2}^{2} y(y-2 z)+m_{3}^{2} z(2 y+3 z)\right) \\
+s^{4} x z\left(-\left(m_{1}^{4}-2 m_{1}^{2}\left(m_{2}^{2}+m_{3}^{2}\right)+\left(m_{2}^{2}-m_{3}^{2}\right)^{2}\right)\right) \\
\times\left(m_{1}^{4} z(2 x-y)\right. \\
+m_{1}^{2}\left(m_{2}^{2}\left(12 x y+10 x z+3 y^{2}+10 y z\right)+m_{3}^{2} z(2 x+2 y+5 z)\right) \\
\left.+\left(m_{2}^{2}-m_{3}^{2}\right)(y+z)\left(3 m_{2}^{2} y+m_{3}^{2} z\right)\right) \\
+s^{2} x z\left(m_{1}^{4} x(24 y+34 z)\right. \\
+m_{1}^{2}\left(m_{2}^{2}\left(12 x y+26 x z+3 y^{2}+32 y z\right)+3 m_{3}^{2}\left(8 x y+6 x z+7 z^{2}\right)\right) \\
\left.\quad+3 m_{2}^{4} y(y-4 z)+m_{2}^{2} m_{3}^{2}\left(9 y^{2}-4 y z-7 z^{2}\right)-5 m_{3}^{4} z^{2}\right) \\
-s^{3} x z\left(2 m_{1}^{6}(6 x y+7 x z+y z)\right. \\
+m_{1}^{4}\left(m_{2}^{2}\left(48 x y+36 x z-3 y^{2}+26 y z\right)\right. \\
\left.\quad+m_{3}^{2}(4 x(z-6 y)+z(19 z-2 y))\right) \\
\quad-2 m_{1}^{2}\left(m_{2}^{4}(x(6 y+z)-15 y(y+z))\right. \\
\quad-m_{2}^{2} m_{3}^{2}\left(24 x y+26 x z-3 y^{2}+2 y z+5 z^{2}\right) \\
\left.\quad+m_{3}^{4}(x(z-6 y)+z(y+z))\right) \\
\quad-\left(m_{2}^{2}-m_{3}^{2}\right)\left(m_{2}^{4} y(3 y+10 z)+m_{2}^{2} m_{3}^{2}\left(9 y^{2}+4 y z+5 z^{2}\right)\right. \\
\left.\left.+m_{3}^{4} z(2 y-z)\right)\right)-x y z^{2} .
\end{aligned}
$$

B.2. The coefficients $\tilde{C}_{x}, \tilde{C}_{y}$ and $\tilde{C}_{z}$. - The coefficients $\tilde{C}_{x}, \tilde{C}_{y}$ and $\tilde{C}_{z}$ are homogeneous polynomials of degree one in $(x, y, z)$ with the detailed expressions given below.

Setting $N=3\left(s^{2} \prod_{i=1}^{4} \mu_{i}-2 s M_{2}+3\right) \prod_{i=1}^{4}\left(\mu_{i}^{2} s-1\right)$ we have for $\tilde{C}_{x}$

$$
\begin{aligned}
2 N \tilde{C}_{x}= & s x\left(55 m_{1}^{2}+43 m_{2}^{2}+49 m_{3}^{2}\right) \\
+ & 2 s^{2}\left(21 m_{1}^{4} x+m_{1}^{2}\left(m_{2}^{2}(52 x+6 y)+6 m_{3}^{2}(6 x+z)\right)+m_{2}^{4} x\right. \\
& \left.+2 m_{2}^{2} m_{3}^{2}(35 x-3(y+z))+9 m_{3}^{4} x\right) \\
+ & s^{5}\left(-\left(m_{1}^{4}-2 m_{1}^{2}\left(m_{2}^{2}+m_{3}^{2}\right)+\left(m_{2}^{2}-m_{3}^{2}\right)^{2}\right)\right) \\
& \times\left(3 m_{1}^{6} x+m_{1}^{4}\left(m_{2}^{2}(5 x+12 y)+3 m_{3}^{2}(x+4 z)\right)\right. \\
& -m_{1}^{2}\left(m_{2}^{4}(7 x+12 y)+22 m_{2}^{2} m_{3}^{2} x+3 m_{3}^{4}(x+4 z)\right) \\
& \left.-\left(m_{2}^{2}-m_{3}^{2}\right)\left(m_{2}^{4} x+2 m_{2}^{2} m_{3}^{2}(x-6 y+6 z)-3 m_{3}^{4} x\right)\right)
\end{aligned}
$$




$$
\begin{aligned}
-2 s^{3} & \left(3 m_{1}^{6} x+m_{1}^{4}\left(m_{2}^{2}(23 x+18 y)+m_{3}^{2}(13 x+18 z)\right)\right. \\
& +m_{1}^{2}\left(7 m_{2}^{4}(5 x-6 y)+70 m_{2}^{2} m_{3}^{2} x+m_{3}^{4}(23 x-42 z)\right)-21 m_{2}^{6} x \\
& \left.+3 m_{2}^{4} m_{3}^{2}(17 x+14 y-6 z)+m_{2}^{2} m_{3}^{4}(49 x-18 y+42 z)-15 m_{3}^{6} x\right) \\
+s^{4} & \left(m_{1}^{8} x-4 m_{1}^{6}\left(m_{2}^{2}(4 x-9 y)-9 m_{3}^{2} z\right)+2 m_{1}^{4}\left(m_{2}^{4}(11 x-28 y)\right.\right. \\
+ & \left.2 m_{2}^{2} m_{3}^{2}(5 x-19(y+z))-m_{3}^{4}(5 x+28 z)\right)+4 m_{1}^{2}\left(m_{2}^{6}(4 x+5 y)\right. \\
+ & \left.m_{2}^{4} m_{3}^{2}(32 x+19 z)+m_{2}^{2} m_{3}^{4}(20 x+19 y)+m_{3}^{6}(8 x+5 z)\right) \\
- & \left(m_{2}^{2}-m_{3}^{2}\right)\left(23 m_{2}^{6} x+m_{2}^{4} m_{3}^{2}(11 x+20 y+36 z)\right. \\
- & \left.\left.m_{2}^{2} m_{3}^{4}(11 x+36 y+20 z)-23 m_{3}^{6} x\right)\right)+21 x
\end{aligned}
$$

for $\tilde{C}_{y}$

$$
\begin{aligned}
N \tilde{C}_{y}= & 7 s y\left(m_{1}^{2}+m_{2}^{2}+m_{3}^{2}\right) \\
+ & s^{2}\left(-6 m_{1}^{4} y+2 m_{1}^{2}\left(m_{2}^{2}(3 x-7 y)-3 m_{3}^{2}(x+2 y+z)\right)\right. \\
& \left.-2\left(m_{2}^{4} y+m_{2}^{2} m_{3}^{2}(7 y-3 z)+3 m_{3}^{4} y\right)\right) \\
+ & s^{5}\left(m_{1}^{4}-2 m_{1}^{2}\left(m_{2}^{2}+m_{3}^{2}\right)+\left(m_{2}^{2}-m_{3}^{2}\right)^{2}\right) \\
\times & \left(3 m_{1}^{6} y+m_{1}^{4}\left(m_{2}^{2}(6 x-y)-3 m_{3}^{2}(2 x+y-2 z)\right)\right. \\
& -m_{1}^{2}\left(m_{2}^{4}(6 x+y)-2 m_{2}^{2} m_{3}^{2} y+3 m_{3}^{4}(-2 x+y+2 z)\right) \\
& \left.-\left(m_{2}^{2}-m_{3}^{2}\right)\left(m_{2}^{4} y+2 m_{2}^{2} m_{3}^{2}(y+3 z)+3 m_{3}^{4} y\right)\right) \\
+ & s^{3}\left(6 m_{1}^{6} y+6 m_{1}^{4}\left(7 m_{2}^{2} x-m_{3}^{2}(7 x+y-3 z)\right)\right. \\
& -2 m_{1}^{2}\left(m_{2}^{4}(9 x-4 y)-28 m_{2}^{2} m_{3}^{2} y+3 m_{3}^{4}(-3 x+y+7 z)\right) \\
& \left.-6 m_{2}^{6} y+2 m_{2}^{4} m_{3}^{2}(4 y-9 z)+42 m_{2}^{2} m_{3}^{4} z+6 m_{3}^{6} y\right) \\
+ & s^{4}+-7 m_{1}^{8} y+2 m_{1}^{6}\left(m_{2}^{2}(5 x+7 y)+m_{3}^{2}(-5 x+10 y-9 z)\right) \\
& -2 m_{1}^{4}\left(m_{2}^{4}(14 x+5 y)+m_{2}^{2} m_{3}^{2}(7 y-19 z)\right. \\
& \left.+m_{3}^{4}(-14 x+13 y-14 z)\right) \\
& +2 m_{1}^{2}\left(m_{2}^{6}(9 x-y)-m_{2}^{4} m_{3}^{2}(19 x+10 y+19 z)\right. \\
& \left.+m_{2}^{2} m_{3}^{4}(19 x-7 y)+m_{3}^{6}(-9 x+10 y-5 z)\right) \\
& +\left(m_{2}^{2}-m_{3}^{2}\right)\left(5 m_{2}^{6} y+3 m_{2}^{4} m_{3}^{2}(y+6 z)-m_{2}^{2} m_{3}^{4}(7 y+10 z)\right. \\
& \left.\left.+7 m_{3}^{6} y\right)\right)-3 y
\end{aligned}
$$


and for $\tilde{C}_{z}$

$$
\begin{gathered}
2 N \tilde{C}_{z}=-s z\left(m_{1}^{2}+13 m_{2}^{2}+7 m_{3}^{2}\right) \\
-2 s^{2}\left(9 m_{1}^{4} z+2 m_{1}^{2}\left(m_{2}^{2}(3 x+3 y-5 z)-3 m_{3}^{2}(x+4 z)\right)\right. \\
\left.-7 m_{2}^{4} z+2 m_{2}^{2} m_{3}^{2}(4 z-3 y)-3 m_{3}^{4} z\right) \\
+s^{5}\left(m_{1}^{4}-2 m_{1}^{2}\left(m_{2}^{2}+m_{3}^{2}\right)+\left(m_{2}^{2}-m_{3}^{2}\right)^{2}\right) \\
\times\left(3 m_{1}^{6} z+m_{1}^{4}\left(m_{2}^{2}(-12 x+12 y+z)+3 m_{3}^{2}(4 x+z)\right)\right. \\
+m_{1}^{2}\left(m_{2}^{4}(12 x-12 y-11 z)+10 m_{2}^{2} m_{3}^{2} z-3 m_{3}^{4}(4 x+z)\right) \\
\left.+\left(m_{2}^{2}-m_{3}^{2}\right)\left(7 m_{2}^{4} z+2 m_{2}^{2} m_{3}^{2}(6 y+z)+3 m_{3}^{4} z\right)\right) \\
+2 s^{3}\left(15 m_{1}^{6} z+m_{1}^{4}\left(m_{2}^{2}(-42 x+18 y-19 z)+m_{3}^{2}(42 x-23 z)\right)\right. \\
+m_{1}^{2}\left(3 m_{2}^{4}(6 x-14 y-5 z)+14 m_{2}^{2} m_{3}^{2} z-m_{3}^{4}(18 x+13 z)\right) \\
\left.+3 m_{2}^{6} z+m_{2}^{4} m_{3}^{2}(42 y+z)+m_{2}^{2} m_{3}^{4}(7 z-18 y)-3 m_{3}^{6} z\right) \\
+s^{4}\left(-17 m_{1}^{8} z-4 m_{1}^{6}\left(m_{2}^{2}(5 x+9 y-9 z)-m_{3}^{2}(5 x+2 z)\right)\right. \\
+m_{1}^{4}\left(m_{2}^{4}(56 x+56 y-38 z)+4 m_{2}^{2} m_{3}^{2}(19 y+14 z)+2 m_{3}^{4}(13 z-28 x)\right) \\
-4 m_{1}^{2}\left(m_{2}^{6}(9 x+5 y-9 z)+m_{2}^{4} m_{3}^{2}(10 z-19 x)\right. \\
\left.\quad+m_{2}^{2} m_{3}^{4}(19 x+19 y+z)+3 m_{3}^{6}(2 z-3 x)\right) \\
-\left(m_{2}^{2}-m_{3}^{2}\right)\left(17 m_{2}^{6} z-m_{2}^{4} m_{3}^{2}(20 y+23 z)\right. \\
\left.\left.\quad+3 m_{2}^{2} m_{3}^{4}(12 y+5 z)+7 m_{3}^{6} z\right)\right)+3 z .
\end{gathered}
$$

\section{References}

[ABW1] L. Adams, C. Bogner and S. Weinzierl, The two-loop sunrise graph with arbitrary masses, J. Math. Phys. 54 (2013) 052303. [arXiv:1302.7004 [hep-ph]].

[ABW2] L. Adams, C. Bogner, and S. Weinzierl, The two-loop sunrise graph in two spacetime dimensions with arbitrary masses in terms of elliptic dilogarithms, J. Math. Phys. 55 (2014) 10, 102301. [arXiv:1405.5640 [hep-ph]].

[ABW3] L. Adams, C. Bogner, and S. Weinzierl, The two-loop sunrise integral around four space-time dimensions and generalisations of the Clausen and Glaisher functions towards the elliptic case, J. Math. Phys. 56 (2015) 7, 072303. [arXiv:1504.03255 [hep-ph]].

[ABW4] L. Adams, C. Bogner, and S. Weinzierl, The iterated structure of the all-order result for the two-loop sunrise integral, J. Math. Phys. 57 (2016) no.3, 032304 [arXiv:1512.05630 [hep-ph]].

[ABW5] L. Adams, C. Bogner, and S. Weinzierl, A walk on sunset Boulevard, PoS RADCOR 2015 (2016) 096 [arXiv:1601.03646 [hep-ph]]. 
[ADKMV] M. Aganagic, R. Dijkgraaf, A. Klemm, M. Mariño, and C. Vafa, Topological strings and integrable hierarchies, Commun. Math. Phys. 261 (2006) 451. [hepth/0312085].

[AKMV] M. Aganagic, A. Klemm, M. Mariño, and C. Vafa, The topological vertex, Commun. Math. Phys. 254 (2005) 425. [hep-th/0305132].

[ABBF] O. Amini, S. Bloch, J. Burgos Gil, and J. Fresan, Feynman amplitudes and limits of heights, Izv. Math. 80 (2016) 813 [arXiv:1512.04862 [math.AG]].

[AM] M. Argeri and P. Mastrolia, Feynman diagrams and differential equations, Int. J. Mod. Phys. A 22 (2007) 4375. [arXiv:0707.4037 [hep-ph]].

[BBDG] D. H. Bailey, J. M. Borwein, D. Broadhurst, and M. L. Glasser, Elliptic integral evaluations of Bessel moments, J. Phys. A 41 (2008) 205203 [arXiv:0801.0891 [hep-th]].

[AM] P. S. Aspinwall and D. R. Morrison, Topological field theory and rational curves, Commun. Math. Phys. 151 (1993) 245. [hep-th/9110048].

[Bat] V. V. Batyrev, Dual polyhedra and mirror symmetry for Calabi-Yau hypersurfaces in toric varieties, J. Alg. Geom. 3 (1994), 493-535. [alg-geom/9310003].

[BW] C. Bogner and S. Weinzierl, Feynman graph polynomials, Int. J. Mod. Phys. A 25 (2010) 2585. [arXiv:1002.3458 [hep-ph]].

[BKV] S. Bloch, M. Kerr, and P. Vanhove, A Feynman integral via higher normal functions, Compos. Math. 151 (2015) 2329. [arXiv:1406.2664 [hep-th]]

[BV] S. Bloch and P. Vanhove, The elliptic dilogarithm for the sunset graph, J. Number Theory 148 (2015), 328-364. [arXiv:1309.5865 [hep-th]].

[B1] F. Brown, Feynman amplitudes and cosmic Galois group, Commun. Num. Theor. Phys. 11 (2017) 453 [arXiv:1512.06409 [math-ph]].

[B2] F. Brown, Periods and Feynman amplitudes, arXiv:1512.09265 [math-ph].

[Chand] K. Chandrasekharan, Elliptic functions, Grundlehren der mathematischen Wissenschaften, A Series of Comprehensive Studies in Mathematics 281, SpingerVerlag (1980).

[CKYZ] T.-M. Chiang, A. Klemm, S.-T. Yau, and E. Zaslow, Local mirror symmetry: calculations and interpretations, Adv. Theor. Math. Phys. 3 (1999) 495 [hepth/9903053].

[C] C. H. Clemens, Degeneration of Kähler manifolds, Duke Math. J. 44 (1977), no. 2, 215-290.

[CK] D. Cox and S. Katz, Mirror symmetry and algebraic geometry, Math. Surveys and Monographs 68, AMS, Providence, RI, 1999.

[DK] C. Doran and M. Kerr, Algebraic K-theory of toric hypersurfaces, CNTP 5 (2011), no. 2, 397-600.

[EH] H. Elvang and Y.t. Huang, Scattering amplitudes, arXiv:1308. 1697 [hep-th].

[Gri] P. A. Griffiths, Periods of certain rational integrals, Ann. of Math. 90 (1969), 460.

[GKPa] S. Groote, J. G. Korner, and A. A. Pivovarov, On the evaluation of a certain class of Feynman diagrams in x-space: Sunrise-type topologies at any loop order, Annals Phys. 322 (2007) 2374. [hep-ph/0506286]. 
[GKPb] S. Groote, J. G. Korner, and A. A. Pivovarov, A numerical test of differential equations for one- and two-loop sunrise diagrams using configuration space techniques, Eur. Phys. J. C 72 (2012) 2085. [arXiv:1204.0694 [hep-ph]].

[Henna] J. M. Henn, Multiloop integrals in dimensional regularization made simple, Phys. Rev. Lett. 110 (2013) 251601. [arXiv:1304.1806 [hep-th]].

[Hennb] J. M. Henn, Lectures on differential equations for Feynman integrals, J. Phys. A 48 (2015) 153001. [arXiv:1412.2296 [hep-ph]].

[HV] K. Hori and C. Vafa, Mirror symmetry, arXiv:hep-th/0002222.

[Hor] K. Hori, S. Katz, A. Klemm, R. Pandharipande, R. Thomas, C. Vafa, R. Vakil, and E. Zaslow, Mirror symmetry, Clay Mathematics Monographs 1, AMS, 2003.

[Hos] S. Hosono, Central charges, symplectic forms, and hypergeometric series in local mirror symmetry, in: Mirror Symmetry V (Lewis, Yau, Yui, eds.), pp. 405-440, AMS/IP Stud. Adv. Math. 38, 2006.

[Ir] H. Iritani, Quantum cohomology and periods, Ann. Inst. Fourier 61 (2011), no. 7, 2909-2958.

[KLM] M. Kerr, J. Lewis, and S. Müller-Stach, The Abel-Jacobi map for higher Chow groups, Compos. Math. 142 (2006), no. 2, 374-396.

[KPR] M. Kerr, G. Pearlstein, and C. Robles, Partial orders and polarized relations on limit mixed Hodge structures, in preparation.

[KOS] R. Kenyon, A. Okounkov, and S. Sheffield, Dimers and amoebae, Ann. Math. 163 (2006), no. 3, 1019-1056. [math-ph/0311005.]

[L] R. N. Lee, Reducing differential equations for multiloop master integrals, JHEP 1504 (2015) 108. [arXiv:1411.0911 [hep-ph]].

[MSWZ] S. Muller-Stach, S. Weinzierl, and R. Zayadeh, A second-order differential equation for the two-loop sunrise graph with arbitrary masses, Commun. Num. Theor. Phys. 6 (2012) 203 [arXiv:1112.4360 [hep-ph]].

[ORV] A. Okounkov, N. Reshetikhin, and C. Vafa, Quantum Calabi-Yau and classical crystals, Progr. Math. 244 (2006) 597. [hep-th/0309208].

[Pari] The PARI Group, PARI/GP version 2.8.0, Bordeaux, 2015, http://pari . math . u-bordeaux.fr/.

[Sc] W. Schmid, Variation of Hodge structure: the singularities of the period mapping, Invent. Math. 22 (1973), 211-319.

[RT] E. Remiddi and L. Tancredi, Schouten identities for Feynman graph amplitudes; The master integrals for the two-loop massive sunrise graph, Nucl. Phys. B 880 (2014) 343. [arXiv:1311.3342 [hep-ph]].

[St1] J. Stienstra, Mahler measure variations, Eisenstein series and instanton expansions, Mirror Symmetry, V, (Banff, AB, 2003), (N. Yui, S.-T. Yau, and J. D. Lewis, eds.) AMS/IP Stud. Adv. Math. 38, Amer. Math. Soc., Providence, RI, 2006, pp. 139-150.

[St2] J. Stienstra, Mahler measure, Eisenstein series and dimers, Mirror Symmetry, V, (Banff, AB, 2003), (N. Yui, S.-T. Yau, and J. D. Lewis, eds.) AMS/IP Stud. Adv. Math. 38, Amer. Math. Soc., Providence, RI, 2006, pp. 151-158. 
[T] L. Tancredi, Integration by parts identities in integer numbers of dimensions. A criterion for decoupling systems of differential equations, Nucl. Phys. B 901 (2015) 282. [arXiv:1509.03330 [hep-ph]].

[Va] P. Vanhove, The physics and the mixed Hodge structure of Feynman integrals, Proc. Symp. Pure Math. 88 (2014) 161. [arXiv:1401.6438 [hep-th]].

[Vo] C. Voisin, A mathematical proof of a formula of Aspinwall and Morrison, Compositio Math., 104 1996, no. 2, 135-151.

March 8, 2018

Spencer Bloch, 5765 S. Blackstone Ave., Chicago, IL 60637, USA

E-mail : spencer_bloch@yahoo.com

Matt KerR, Department of Mathematics, Campus Box 1146, Washington University in St. Louis, St. Louis, MO, 63130, USA • E-mail : matkerr@math. wustl.edu

Pierre Vanhove, Department of Applied Mathematics and Theoretical Physics, Wilberforce Road, Cambridge CB3 0WA, UK

Institut de physique théorique, Université Paris Saclay, CEA, CNRS, F-91191 Gif-sur-Yvette E-mail : pierre.vanhove@cea.fr 